Check for updates

Cite this: J. Mater. Chem. C, 2020, 8, 1517

Received 17th June 2019,

Accepted 30th November 2019

DOI: $10.1039 / \mathrm{c} 9 \mathrm{tc0} 3251 \mathrm{~g}$

rsc.li/materials-c

\section{Harvesting graphene oxide - years 1859 to 2019: a review of its structure, synthesis, properties and exfoliation}

\begin{abstract}
P. P. Brisebois and M. Siaj (D)*
In recent years, multilayered graphite oxide and graphene oxide (GO) have attracted considerable attention in fields such as physics, chemistry, biology and materials sciences in general, because they are important building blocks and promising routes towards the large-scale production of graphene, the "wonder material". This review provides an exhaustive survey of the synthetic methods developed during the last 160 years for the preparation of GO and focuses especially on the work done more recently. The numerous state-of-the-art synthetic methods (>50) are reviewed and regrouped under eight (8) major categories: those which use oxidative (i) chemical, (ii) electrochemical and (iii) microbial exfoliation methods for graphite, for (iv) 3D-carbon structures and for (v) 2D-graphene. Other routes such as (vi) chemical vapor deposition (CVD) methods for hydrocarbon, (vii) hydrothermal methods for carbohydrate and finally, (viii) thermal decomposition methods for organic matter rich in carbon are also covered. Emphasis is placed on the molecular structure of graphene oxide, synthetic methods, impurities, properties, mechanistic insights, reagents, different carbon precursors, its purification, the exfoliation process and finally, its fractionation by size. This review will be a valuable guide $(>200$ references) for synthetic and materials scientists/engineers in the field of graphene oxide production.
\end{abstract}

Department of Chemistry, Université du Québec à Montréal (UQÀM)/NanoQÀM-CQMF, 2101, Jeanne-Mance street, Montreal, QC, H2X 2J6, Canada. E-mail: siaj.mohamed@uqam.ca; Fax: +1 (514) 987-4054; Tel: +1 (514) 987-3000 ext. 1921

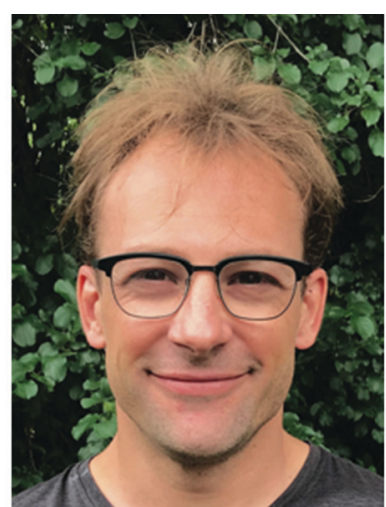

P. P. Brisebois
Dr Patrick Brisebois is currently a postdoctoral researcher in the Department of Physics at McGill University (Canada). He did his doctoral work in the Department of Chemistry under the guidance of Prof. Siaj and he received his $P h D$ degree from Université $d u$ Québec à Montréal (UQÀMCanada). He was awarded " $F Q$ $R N T$ " multidisciplinary scholarships twice by the government of Québec for both his doctoral and postdoctoral research in 2013 and 2017. His interests focus on the synthesis of carbon materials and their nano- to macro-scale assemblies. At present, he is working on 3D-CVD graphene microelectrodes for highfrequency electrophysiological applications.

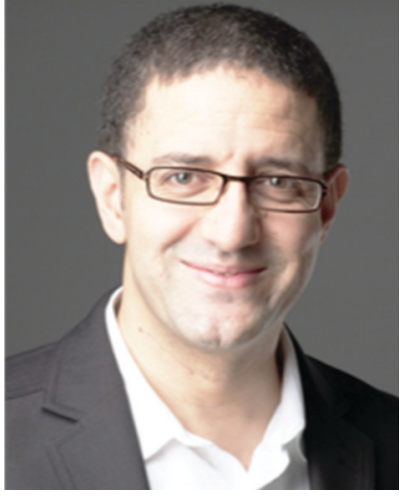

M. Siaj
Prof. Mohamed Siaj received his PhD from Université Laval (Canada) under the guidance of Prof. Peter McBreen. Following postdoctoral training at the Colin Nuckolls group at Columbia University, New York, a leading institution in 2D Materials based science, Siaj joined the Department of Chemistry at UQAM as an Assistant Professor in 2008, and now holds the rank of a Full Professor. He is a Tiers II Canada Research Chair in 2D-materials for sensing applications. At present, Prof. Siaj is the director of NanoQÀM (Canada) for nanomaterials and energy. He has a broad interest in synthesis, characterization and application of new $2 D$ materials and their integration into electrodes for chemical and biosensor applications. 


\section{Introduction}

In 2019, planning to review the synthetic methods for the preparation of graphene oxide (GO) is quite challenging because excellent books ${ }^{1-3}$ and reviews ${ }^{4-8}$ have already been published in the last few years. A considerable amount of planning and literature search was necessary to avoid repeating the information so well retrieved by others who focused primarily on the history of the preparation of GO, the mechanism of the oxidative chemical exfoliation of graphite ( $\mathrm{Gr}$ ), the characterization of GO, its properties, its scope of applications and its chemical functionalization. Despite the efforts of other reviewers to describe and compare the early structural models of GO from a historical point of view, in our opinion, none of them were able to overview all the aspects of its structure with accuracy in accordance with the fundamental aspects of carbon chemistry because they failed to point out the fact that the models share a common flaw: according to the octet chemical rule of thumb, each carbon atom should make four bonds with its surrounding. Unfortunately, every structural model (Fig. 1) that has been proposed or reviewed over the last 80 years shows implicit $\mathrm{CH}$ and $\mathrm{CH}_{2}$ groups by omitting to draw the hydrogen atoms in the model and none of the reviews have reported any explanation or alternative to this omission or have reported inclusion of such $\mathrm{C}-\mathrm{H}$ bonds in their model (Fig. 1-red circles). This fundamental aspect can be useful to clarify the chemical nature of GO, especially for multidisciplinary researchers in cross-disciplines who are not experts in chemistry, therefore it needs an explication. Perhaps, the answer to this is that researchers just don't know if hydrogen attached to carbon atoms are present in GO.
Hydrogen content is very low in GO and most of the characterization techniques are not sensitive enough to detect it directly with accuracy. Also, the presence of carbon radicals (seven-electron species) stabilized by conjugation with the $\pi$ system could also give some part of the explanation for the missing elements. Considering these aspects, herein, we present a useful complement to the fundamental aspects of GO concerning its structure and chemistry.

First, we present a structural account for a nanosheet of graphene oxide (Fig. 2) based on earlier and more modern models ${ }^{1-12}$ and updated with new claims that have been supported more recently with strong experimental evidence from other top researchers. ${ }^{13-22}$ Our account illustrates most of the acknowledged observations made on the structure of GO with a $\mathrm{C} / \mathrm{O}$ ratio of $\sim 2$, including the presence of carbon vacancies, ${ }^{17}$ sulfate esters, ${ }^{18,19}$ carbon radicals, ${ }^{20} \mathrm{C}-\mathrm{H}$ bonds, ${ }^{21}$ and allylic alcohols, ${ }^{22}$ regardless of the fact that most of these aspects (A-P) were not intended initially to explain the same model. GO is a complex macromolecule and different models are used to explain its structure with contradictions, a dazzling confusion. Our structural account is proposed to facilitate the integration of knowledge because all the possible functionalities are fitted in the same frame composed of 74 atoms of carbon, 38 atoms of oxygen, 29 atoms of hydrogen and 1 sulfur atom.

In the core of this review, we present the principal chemical and physical characteristics of GO with a focus on the fundamentals of stacking and interactions of GO in the solid state and in colloidal suspensions. Also, we adopt a benchmark approach by highlighting the relationships between methodschemical composition-properties of GO, with special attention
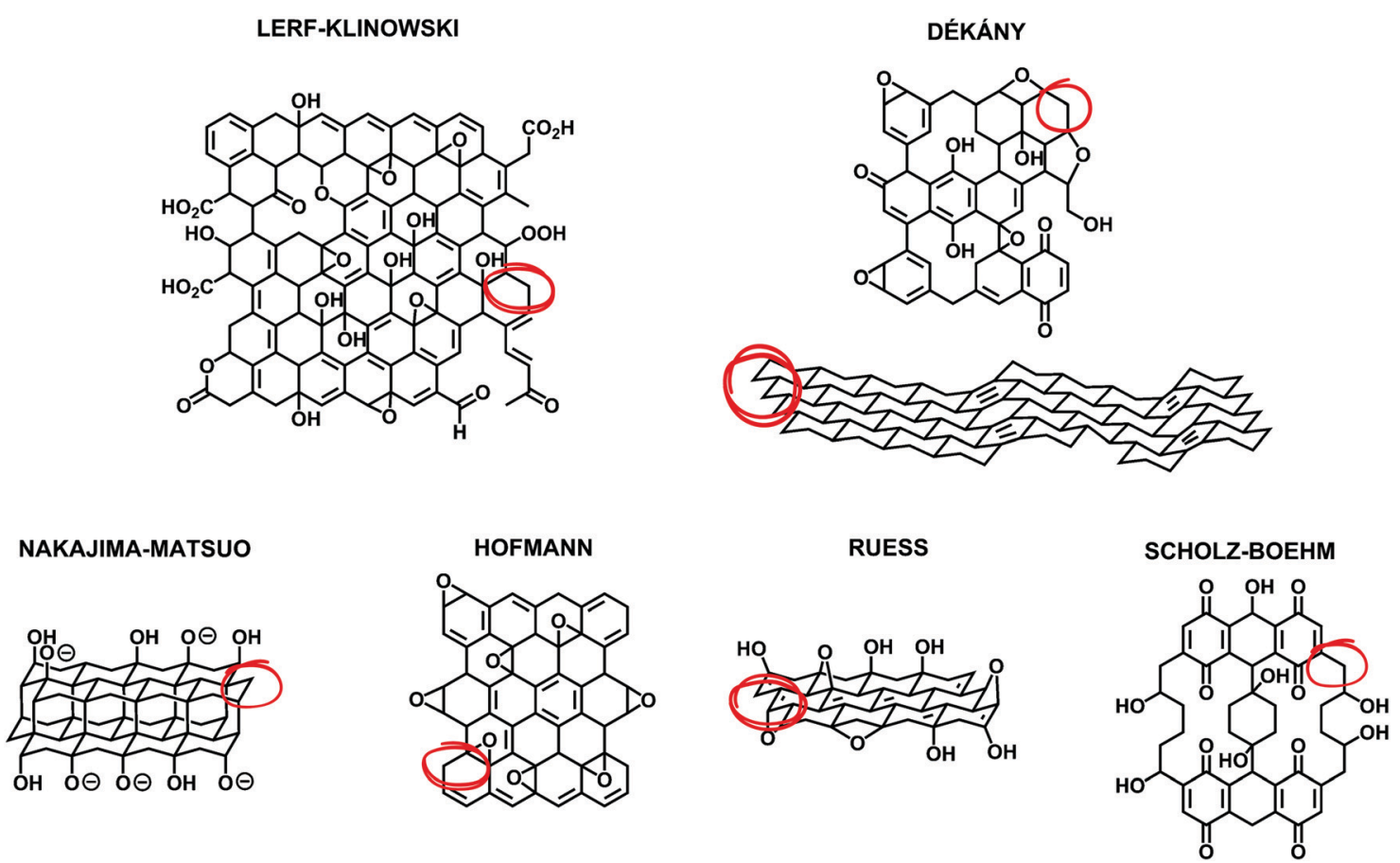

Fig. 1 Summary of structural models of GO that have been proposed, including modern examples (top: Lerf-Klinowski and Dékány models) as well as earlier examples (bottom: Nakajima-Matsuo, Hofmann, Ruess, and Scholz-Boehm models). Reproduced (adapted) from ref. 6 with permission from the Royal Society of Chemistry. 


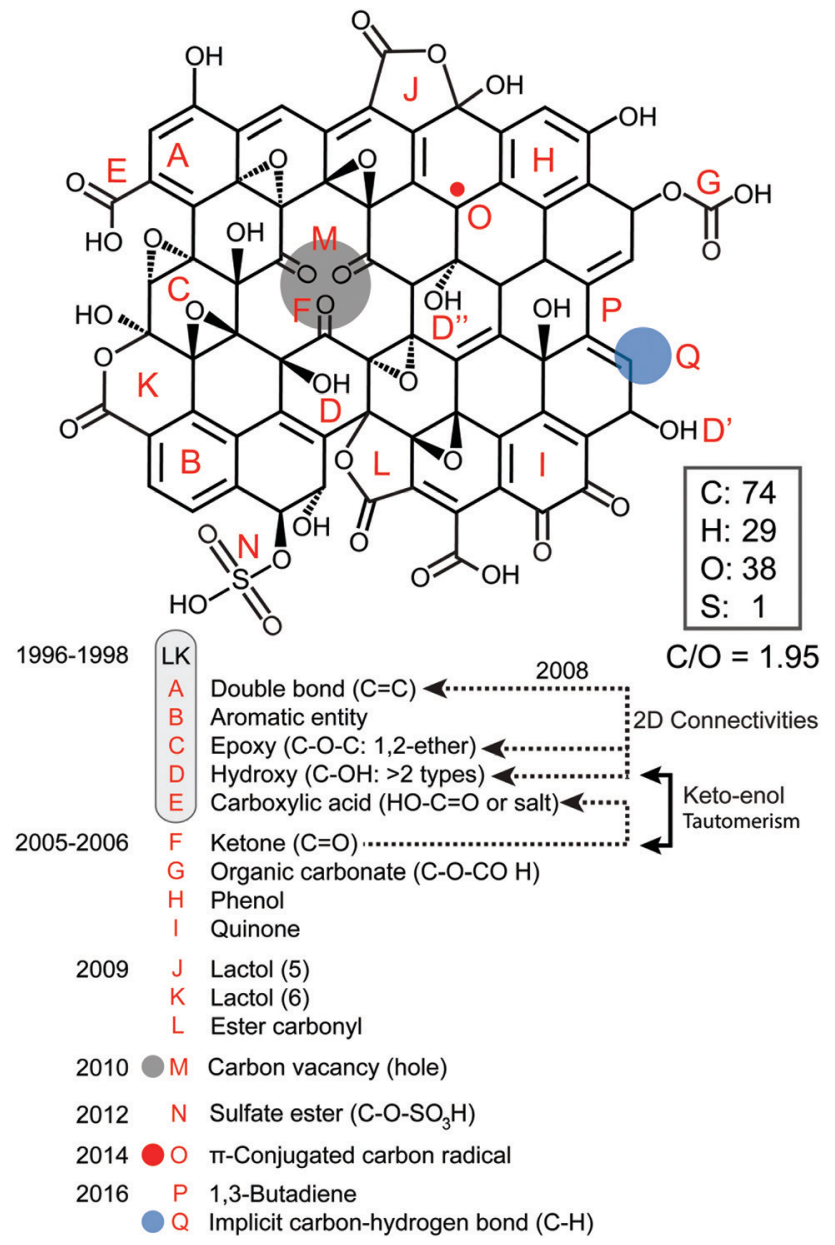

Fig. 2 Structural account of a nanosheet of graphene oxide (GONs) with a $\mathrm{C} / \mathrm{O}$ ratio of $\sim 2$

given to oxygen atoms, heteroatoms and metallic impurities. The methods are regrouped into two useful lists of chemicals. The first for covering the aspects of the oxidative exfoliation of graphite that have been published in the literature in the last 160 years, with special attention to the ones that have not been included by other reviewers. ${ }^{1-8}$ Then, the second list is to cover the synthesis from other carbon precursors besides graphite and novel methods towards GO that have been developed recently, mostly in the last decade. Overall, we present a total of more than 50 original ways to prepare graphene oxide-based materials from the nanogram to the multigram scale. The data are presented in two (2) tables that report some physical properties of GO such as the morphological shape, the number of layers (\#), the lateral size of the sheets and the $\mathrm{C} / \mathrm{O}$ ratio of the materials (when available). Characterization data such as solid state nuclear magnetic resonance (SS-NMR), scanning and transmission electron microscopy (TEM, SEM), thermogravimetric analysis (TGA), X-ray photo emission spectroscopy (XPS) and atomic force microscopy (AFM) are presented, not in a separate section, but in integration all along the review as a way to illustrate and to support scientifically the claims made on GO case by case. The final section is devoted to bringing the most recent advances in the post-synthesis physical treatments used for complete exfoliation of GO to monolayers, for its purification and its fractionation by size. Moreover, techniques such as acoustic cavitation/sonication, shearing stress, freezedrying, micro bubbling, sedimentation, centrifugation, filtration and fractionation of GO by size are described to help the chemist harvesting monolayers of $\mathrm{GO}$ with yield, purity. This guide is very valuable from a synthetic point of view, especially for tailoring GO and targeting specific applications.

\section{Structural accounts for graphene oxide}

\subsection{Lerf-Klinowski model}

The popular model that accounts for most of the experimental observations about GO prepared from the oxidative chemical exfoliation of graphite using potassium permanganate as an oxidizer was proposed by Lerf-Klinowski (LK) in the late 1990s (Fig. 1). ${ }^{9-12}$ Using mostly carbon-13 and hydrogen-1 solid state nuclear magnetic resonance (SSNMR), Fourier transform infrared spectroscopy (FTIR) and chemical derivatization of graphite oxide with reagents having a well-known mechanism of action, LK concluded that the structure of GO contains two kinds of regions: regions with aliphatic six-membered rings and aromatic regions with non-oxidized benzene rings. The relative size of the two regions depends on the degree of oxidation of the compound. As shown in Fig. 2, the surface of the monolayer is composed mainly of double bonds (A), aromatic entities (B) and epoxide groups (C) which give rise to a nearly flat carbon grid; only carbon atoms attached to hydroxy groups have a slightly distorted tetrahedral configuration, resulting in some wrinkling of the monolayer. The oxo functionalities lie above and below the carbon grid forming a layer of oxygen atoms of variable concentrations with a large number of epoxide (1,2-ether) (C) and $\mathrm{C}-\mathrm{OH}\left(\mathrm{D}, \mathrm{D}^{\prime}, \mathrm{D}^{\prime \prime}\right)$ groups (more than two types) very close to one another. It is not necessary for the distribution of functional groups in every oxidized aromatic ring to be identical, and both the oxidized rings and aromatic entities are distributed randomly. The structure of $\mathrm{GO}$ terminates with $\mathrm{C}-\mathrm{OH}$ (D') and $\mathrm{COOH}$ (E) groups, which could also explain the acidity of the compound. More recent experiments based on chemical derivatization using a Claisen-type rearrangement ${ }^{22}$ was also used to show the presence of allylic alcohol $\left(\mathrm{D}^{\prime \prime}\right)$ on the surface of graphite oxide. ${ }^{13} \mathrm{C}$-labeled GO with modern multidimensional SS-NMR methods provided new information on the bonding arrangements of atoms and their $2 \mathrm{D}$ connectivities. ${ }^{13}$ Cai and collaborators observed a strong peak correlation (Fig. 3) between ${ }^{13} \mathrm{C}$-sp ${ }^{2}$ (131 ppm), ${ }^{13} \mathrm{C}$-epoxide (60 ppm) and ${ }^{13} \mathrm{C}-\mathrm{OH}$ (70 ppm) groups which gives more direct evidence that a large fraction of those atoms was chemically bonded to each other, in accordance with the LK model.

\subsection{Dékány model}

Later on, other important aspects of the structure of GO were described by Dékány and collaborators ${ }^{14,15}$ who identified with 

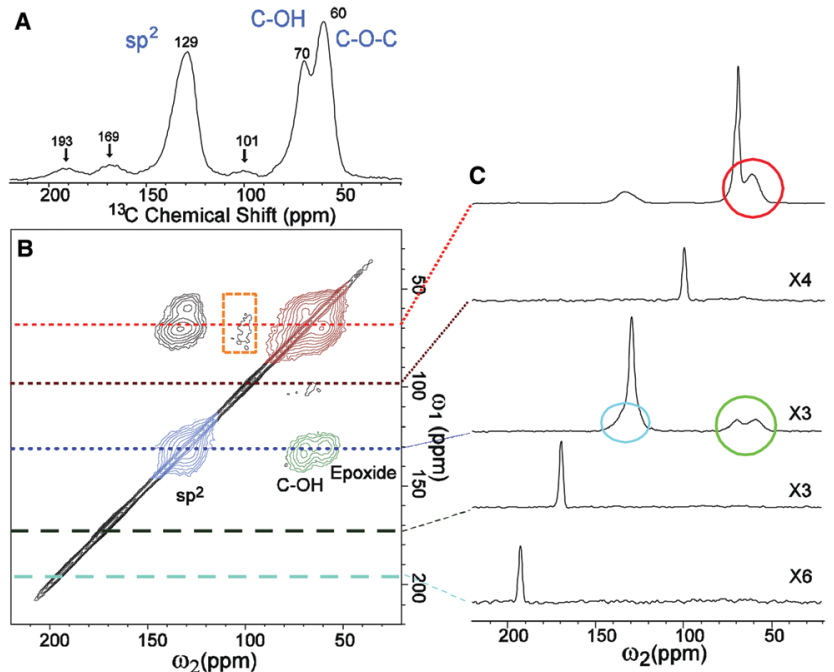

Fig. 3 (A) $1 \mathrm{D}^{13} \mathrm{C}$-MAS and (B) $2 \mathrm{D}{ }^{13} \mathrm{C} /{ }^{13} \mathrm{C}$ chemical-shift correlation solid state NMR spectra of ${ }^{13} \mathrm{C}$-labeled graphite oxide with (C) slices selected from the 2D spectrum at the indicated positions $(70,101,130,169$, and $193 \mathrm{ppm})$ in the $\omega_{1}$ dimension. All the spectra were obtained at a ${ }^{13} \mathrm{C}-\mathrm{NMR}$ frequency of $100.643 \mathrm{MHz}$ with $90 \mathrm{kHz}{ }^{1} \mathrm{H}$ decoupling and $20 \mathrm{kHz}$ MAS for $12 \mathrm{mg}$ of the sample. In (A), the ${ }^{13} \mathrm{C}$-MAS spectrum was obtained with direct ${ }^{13} \mathrm{C}$ excitation by a $\pi / 2$-pulse. The recycle delay was $180 \mathrm{~s}$, and the experimental time was 96 min for 32 scans. In (B), the 2D spectrum was obtained with cross polarization and fpRFDR ${ }^{13} \mathrm{C}-{ }^{13} \mathrm{C}$ dipolar recoupling sequence. The experimental time was $12.9 \mathrm{~h}$ with recycle delays of $1.5 \mathrm{~s}$ and 64 scans for each real or imaginary $t_{1}$ point. A Gaussian broadening of $10 \mathrm{~Hz}$ was applied. The green, red, and blue areas in (B) and circles in (C) represent cross peaks between $\mathrm{sp}^{2}$ and $\mathrm{C}-\mathrm{OH} / \mathrm{epoxide}$ (green), those between $\mathrm{C}-\mathrm{OH}$ and epoxide (red), and those within $\mathrm{sp}^{2}$ groups (blue), respectively. From [Science, 2008, 321, 1815-1818]. Reprinted with permission from AAAS.

no doubt the presence of other oxo functionalities such as a ketone (F), organic carbonate (G), phenol (H) and quinone (I). In their original reports, Dékány and collaborators proposed a modified version of the Scholz-Boehm model to support their new observations. Noteworthily, in their work, GO was prepared using a different method (potassium chlorate oxidation), and hence, had a different chemical composition. Both models are considered plausible, but Dékány's is more specific for GO with a higher level of oxidation, while LK's is more general and suitable for most types of GO. ${ }^{13,16}$ There are major discrepancies between the two models. But one is based on how GO terminates (hydroxy or ketone). Ketone and enol are said to be tautomers of each other and their interconversion involves the movement of an $\alpha$-hydrogen and the shifting of bonding electrons, which depends on many factors such as the $\mathrm{pH}$, the amount of water trapped inside GO and the temperature. Carbon-13 $\left({ }^{13} \mathrm{C}\right)$ solid state nuclear magnetic resonance spectra $\left({ }^{13} \mathrm{C}-\mathrm{SS}-\mathrm{NMR} / \mathrm{MAS}\right)$ of GO (Fig. 4) have poor signal-to-noise ratios, which can explain why LK, back in the 1990s, did not explicitly detect the presence of minor constituents like ketone or enol. In that context, it is plausible to include both $\left(\mathrm{F}, \mathrm{D}^{\prime \prime}\right)$ in the model to terminate GO. Furthermore, Cai and collaborators observed no visible cross peaks between ${ }^{13} \mathrm{C}-\mathrm{sp}^{2}$ (129 ppm) and the other minor components at 169 and $193 \mathrm{ppm}$
Cross Polarization (CP)

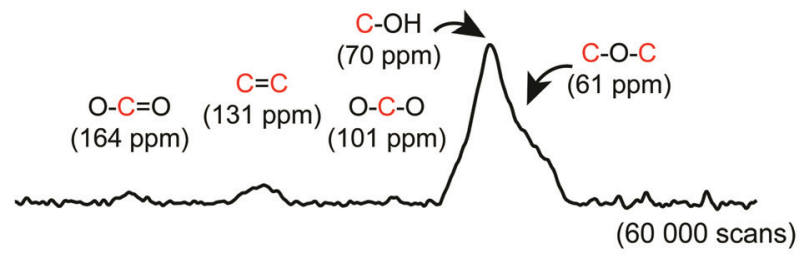

Direct Pulse (DP)

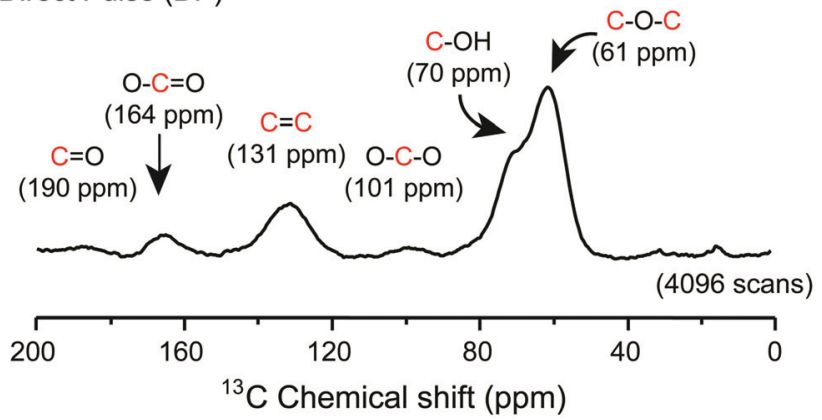

Fig. $4{ }^{1} \mathrm{H}-{ }^{13} \mathrm{C}$ cross-polarization (CP) and direct pulse (DP) ${ }^{13} \mathrm{C}-{ }^{1} \mathrm{H}$ decoupled SS-NMR spectra of graphene oxide. ${ }^{13} \mathrm{C}$-SS-NMR/MAS spectra were recorded at frequencies of $150.874 \mathrm{MHz}$ for ${ }^{13} \mathrm{C}$ and $599.84 \mathrm{MHz}$ for ${ }^{1} \mathrm{H}$ using a $4 \mathrm{~mm}$ magic-angle spinning (MAS) double resonance probe and a zirconium oxide rotor for NMR analysis. Magic angle spinning was performed at a spinning frequency of $12.5 \mathrm{kHz}$. Typically, $80 \mathrm{mg}$ of dry GO material was used in the sample and 60000 and 4096 scans were recorded respectively for $\mathrm{CP}$ and $\mathrm{DP}$ to achieve the desired spectral resolution. All spectra were collected at room temperature $\left(23^{\circ} \mathrm{C}\right)$. Data were analyzed using the Mestrenova ${ }^{\circledR}$ software (Mestrelab research). Exponential line broadening functions of $50 \mathrm{~Hz}$ were applied to the MAS spectra and chemical shifts were referenced relatively to adamantane (38.25 ppm). Cross-polarization- ${ }^{13} \mathrm{C}$-SS-NMR/MAS experiments were carried out using a spectral width of $75 \mathrm{kHz}$, radio-frequency fields of $75 \mathrm{kHz}$ for ${ }^{13} \mathrm{C}$ and $87.5 \mathrm{kHz}$ for ${ }^{1} \mathrm{H}$ with a $30 \%$ ramp, a $500 \mu$ s contact time, a $3 \mathrm{~s}$ recycle delay and an acquisition time of $20 \mathrm{~ms}$. Direct pulse- $-{ }^{13} \mathrm{C}-\mathrm{SS}-$ NMR/MAS spectra were recorded using a broadband proton decoupling at a RF field of $87.5 \mathrm{kHz}$ during acquisition, with a spectral width of $75 \mathrm{kHz}$, a $90^{\circ}$ pulse length of $3.3 \mu \mathrm{s}$, a $20 \mathrm{~s}$ recycle delay and an acquisition time of $20 \mathrm{~ms}$. Reproduced from ref. 21 with permission from Wiley-VCH.

$\left({ }^{13} \mathrm{COOH},{ }^{13} \mathrm{C}=\mathrm{O}\right)$. These results imply that carboxyl (E) and carbonyl (F) groups are spatially separated from a majority of the carbon atoms attributed to the functionalities $(\mathrm{A}, \mathrm{C}, \mathrm{D})$ found on the surface. Therefore, in Fig. 2, carboxylic acid (E) and ketone (F) groups are located on the periphery of GO, terminating the edge of the flake or the grid-hole (M). Important insights into the GO structure were revealed also by Gao and collaborators. ${ }^{16}$ Based on SS-NMR and cross polarization techniques, they were able to identify the presence of other minor components: non-protonated carbon species around $100 \mathrm{ppm}$. According to them: "appropriately substituted five (5)- and six (6)-memberedring lactols (J, K) along the periphery are the most likely structures in GO to be responsible for this signal." In addition, they were also able to identify ester carbonyl (L) signals at $167 \mathrm{ppm}$. They explain their origin from the presence of numerous tertiary alcohols in GO that allows for the possibility of some of them to react with nearby carboxylic acids to form ester groups on the periphery, either on the same GO sheet or an adjacent sheet. 
The full chemistry of GO is not fully understood, and the general LK model should also be updated with important aspects previously unknown in the structure of GO such as carbon vacancies (M), sulfate ester $(\mathrm{N}), \pi$-conjugated carbon radicals $(\mathrm{O})$, implicit $\mathrm{C}-\mathrm{H}$ bonds (P) and 1,3 butadiene systems (Q), which would better describe its chemical reactivity.

\subsection{Structural holes}

Using high resolution transmission electron microscopy (TEM) with a quasi-atomic resolution, experimental observations showed not only that its local chemical structure is composed of two kinds of regions (graphitic and oxidized regions), the way LK described, but also that holes (M) under $5 \mathrm{~nm}^{2}$ are found throughout the sheet (Fig. 5 and 6). This is explained from the release of $\mathrm{CO}$ and $\mathrm{CO}_{2}$ during the aggressive oxidation and sheet exfoliation. Supported by XPS analysis, observations showed that carbonyl (F) groups likely form at the edges of the observed holes within the GO sheet. ${ }^{17}$

\subsection{Organosulfates}

Also, Tour and collaborators have shown that covalent sulfate groups are formed by the nucleophilic attack of sulfuric acid or hydrogen sulfate at newly formed epoxide groups (C) in the course of graphite oxidation using Hummers' method (Scheme 1). Because sulfate ester groups (N) hydrolyze only very slowly, they remain in the chemical structure of GO. ${ }^{18,19}$ TGA-MS data show that a weight loss of about $15 \%$ that is due to $\mathrm{SO}_{2}$ formation and the low formation temperature $\left(200-300{ }^{\circ} \mathrm{C}\right)$ of $\mathrm{SO}_{2}$ is consistent with the decomposition of organosulfates (Fig. 7). ${ }^{19}$

\subsection{Radicals}

Although these functionalities $(\mathrm{A}-\mathrm{N})$ reasonably interpret the individual physical properties of GO such as its acidity and basicity, and its electric, reductive and hydrophilic features, they cannot provide an appropriate understanding of some experimental results, such as the ability of GO to trigger chemiluminescence of luminol for example. Using electron paramagnetic resonance (EPR), Yang and collaborators were able to detect a large number of $\pi$-conjugated carbon radicals (O) at the $\pi$-network plane when GO was freshly prepared. They explained it by the action of hydrogen peroxide used to neutralize
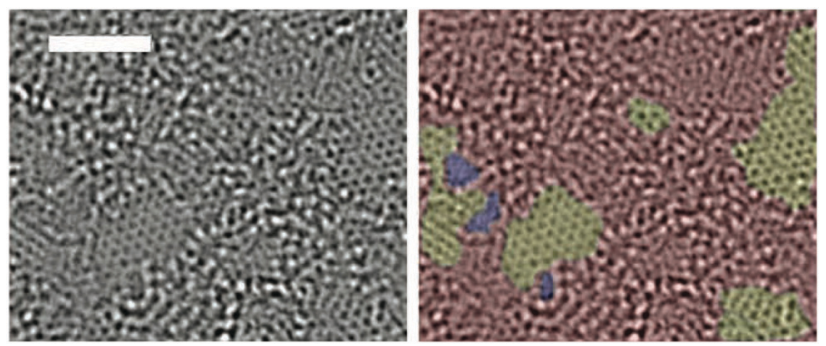

Fig. 5 Aberration-corrected TEM images of a single suspended sheet of GO. Scale bar, denoting two (2) nanometers, is valid for all images. On the right, holes are indicated in blue, graphitic areas in yellow, and high contrast, disordered regions, indicating oxygen functionalities, in red. Reproduced (adapted) from ref. 17 with permission from Wiley-VCH.

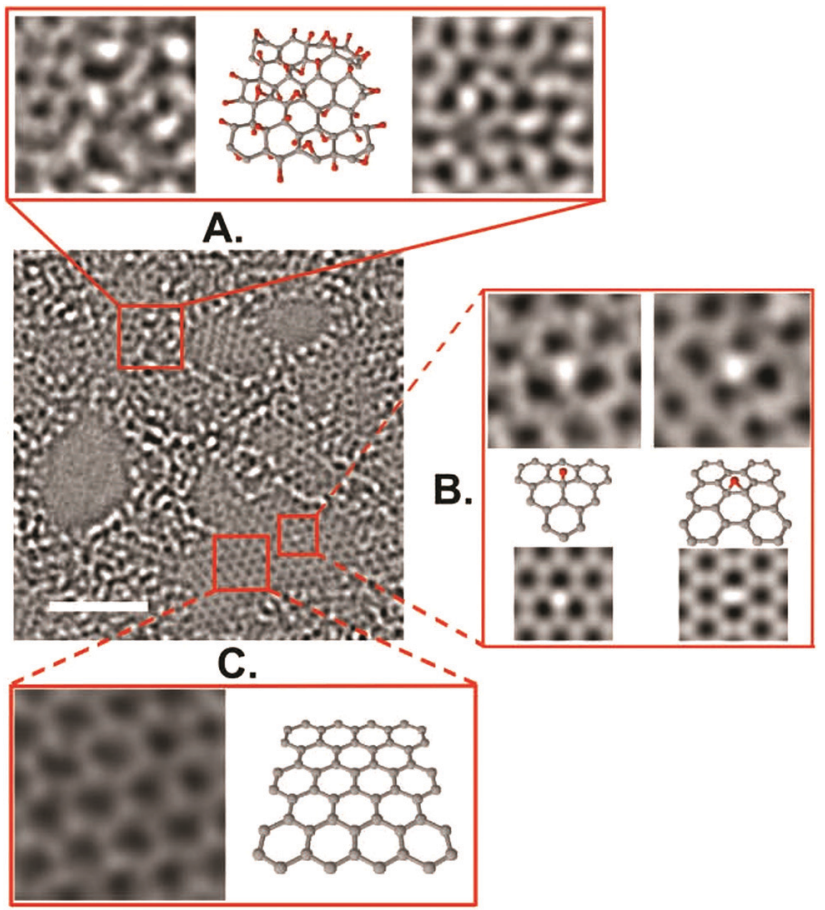

Fig. 6 Aberration-corrected TEM image of a single sheet of suspended GO. The scale bar is $2 \mathrm{~nm}$. Expansion (a) shows, from left to right, a $1 \mathrm{~nm}^{2}$ enlarged oxidized region of the material, then a proposed possible atomic structure of this region with carbon atoms in gray and oxygen atoms in red, and finally the average of a simulated TEM image of the proposed structure and a simulated TEM image of another structure where the position of oxidative functionalities has been changed. Expansion (b) focuses on the white spot on the graphitic region. This spot moved along the graphitic region but stayed stationary for 3 frames ( 6 seconds) at a hydroxyl position (left portion of expansion (b)) and for 7 frames (14 seconds) at a $(1,2)$ epoxy position (right portion of expansion (b)). The ball-and-stick figures below the microscopy images represent the proposed atomic structure for such functionalities. Expansion (c) shows a $1 \mathrm{~nm}^{2}$ graphitic portion from the exit plane wave reconstruction of a focal series of GO and the atomic structure of this region. Reproduced from ref. 17 with permission from Wiley-VCH.

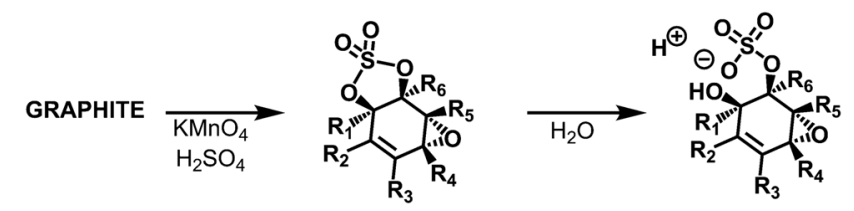

Scheme 1 Organosulfates present in GO prepared via the Hummers' method have been shown to be susceptible to hydrolysis, leading to the formation of acidic sulfate species in the final product. Reproduced from ref. 6 with permission from the Royal Society of Chemistry.

the excess of permanganate ions during the work-up. $\mathrm{H}_{2} \mathrm{O}_{2}$ also reacts with GO by the addition of hydroxyl radicals to the double bonds of the disrupted $\pi$-network plane. The carbon radicals are temporarily stabilized by conjugation, giving a strong non-splitting EPR signal (Fig. 8). ${ }^{20}$

\subsection{Conjugated double bonds}

Using chemical derivatization (Diels-Alder (DA) reaction) and ${ }^{13} \mathrm{C}-\mathrm{SS}-\mathrm{NMR}$, good experimental evidence was obtained for a 


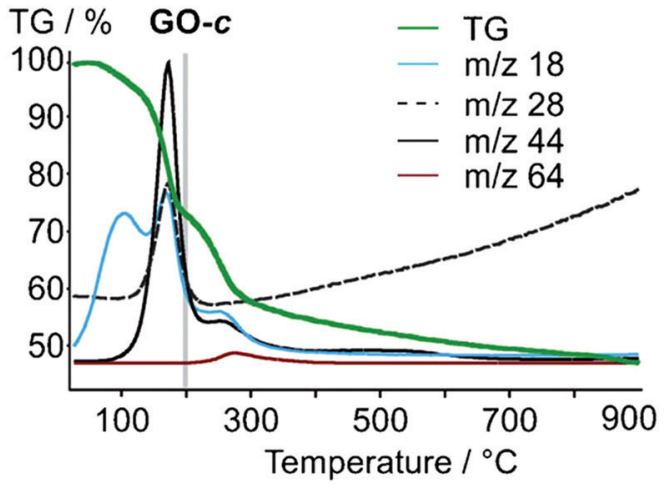

Fig. 7 TGA-MS analysis of GO; $\mathrm{m} / z$ 18, $\mathrm{H}_{2} \mathrm{O} ; \mathrm{m} / \mathrm{z} 28, \mathrm{CO} ; \mathrm{m} / \mathrm{z} 44, \mathrm{CO}_{2}$; $\mathrm{m} / \mathrm{z} 64, \mathrm{SO}_{2}$. Reproduced from ref. 19 with permission from Wiley- $\mathrm{VCH}$.

A

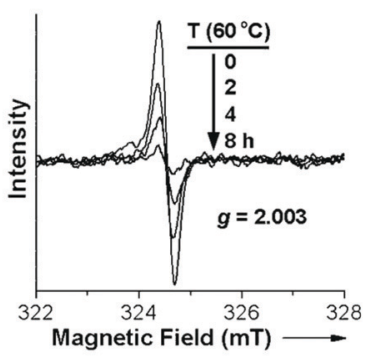

B

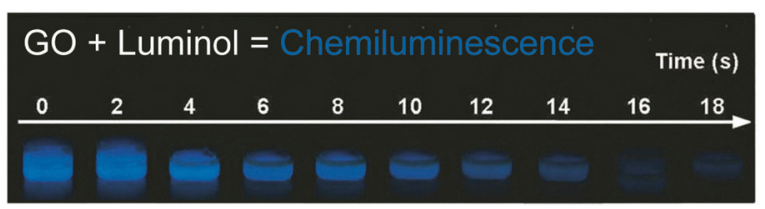

C

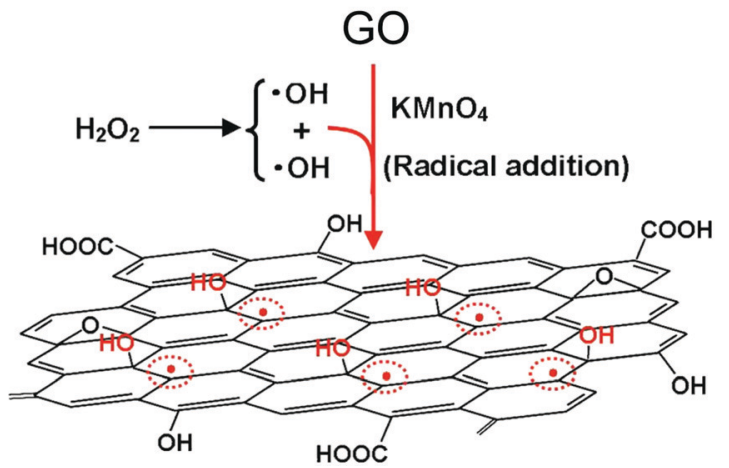

Fig. 8 (A) The time-dependent evolution of the EPR spectrum of GO $\left(0.24 \mathrm{mg} \mathrm{mL}^{-1}\right)$ at an elevated temperature of $60{ }^{\circ} \mathrm{C}$, (B) the strong and longlasting visible chemiluminescence produced by the addition of luminol to a GO solution and (C) the proposed mechanism for the formation of $\pi$-conjugated carbon radicals by the addition of hydroxyl radicals. Reproduced (adapted) from ref. 20 with permission from Wiley-VCH.

[4+2] cycloaddition between GO and maleic anhydride (Scheme 2). ${ }^{21}$ This reopens the fundamental question of $\mathrm{LK}$, about the presence or not of conjugated double bonds in the structure of GO. ${ }^{9}$ According to experimental data, only 1,3-butadiene systems (P) substituted with implicit hydrogen atoms (C-H bonds) (Q) located at the edge of the flake or close to a grid-hole (M) can explain the new signal observed in the alkane region at $30 \mathrm{ppm}$

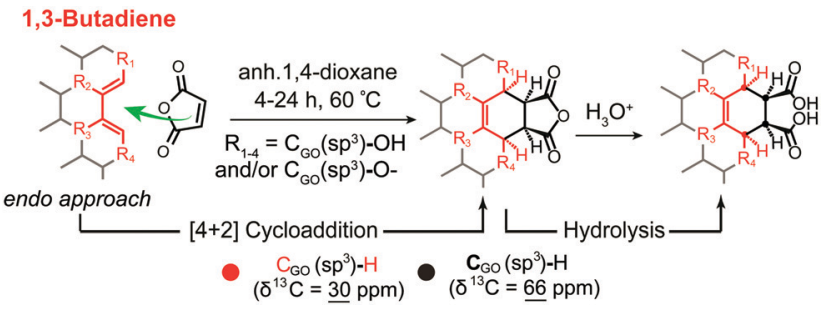

Scheme 2 Proposed mechanism and product structures for the DielsAlder reaction between a 1,3-butadiene system located at the edge of GO and maleic anhydride. Reproduced from ref. 21 with permission from Wiley-VCH.

in the ${ }^{13} \mathrm{C}$-SS-NMR spectrum after the DA reaction. This indirectly proves the existence of protonated carbons in the structure of GO. Therefore, an updated LK model of GO should include this new aspect that explains in a better way how GO terminates in accordance with the octet rule.

\subsection{Allylic oxygen}

The covalent functionalization of soluble, exfoliated graphite oxide through a Claisen rearrangement (Scheme 3$)^{22}$ shows the evidence of the allylic alcohol functional groups found on the surface of GO. Allylic alcohol groups are converted into vinyl allyl ethers when heated with the vinyl transfer reagent $\mathrm{N}, \mathrm{N}$ dimethylacetamide dimethyl acetal (DMDA), and allylically transposed in a sigmatropic-type fashion to form new carboncarbon bonds. As a direct result, robust carboxylate groups are installed on the surface by saponification, which can undergo subsequent synthetic manipulations. ${ }^{22}$

\section{Chemical and physical properties of graphene oxide}

\subsection{Oxygen groups}

Graphene oxide is a carbon-based amorphous compound with a two-dimensional (2D) structure containing oxo functionalities and hydrogen atoms. GO has a non-stoichiometric general formula of the type $\mathrm{C}_{x} \mathrm{H}_{y} \mathrm{O}_{z}$. On average, the amount of hydrogen in the formula is estimated to be $y=0.8$ and the carbon-to-oxygen $(\mathrm{C} / \mathrm{O})$ atomic ratio $(x / z)$ can vary generally from $1.5-2.5(z=1) .{ }^{23}$ However, some authors have reported lower and higher values. $^{24,25}$ The atomic composition of GO can be determined by energy dispersive spectroscopy (EDS), combustion elemental analysis and high-resolution X-ray photoelectron spectroscopy (XPS).

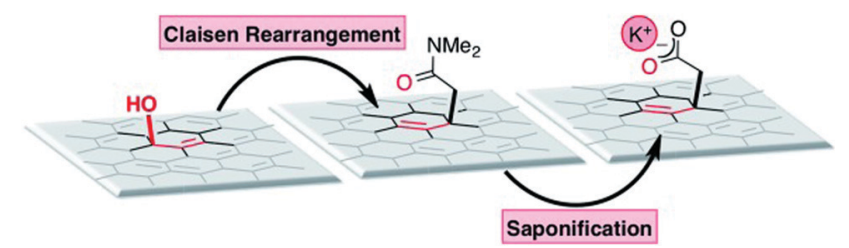

Scheme 3 Allylic oxygen to carbon bond transposition on graphite oxide (additional substrate oxygen groups have been removed for clarity). Reproduced from ref. 22 with permission from Wiley-VCH. 


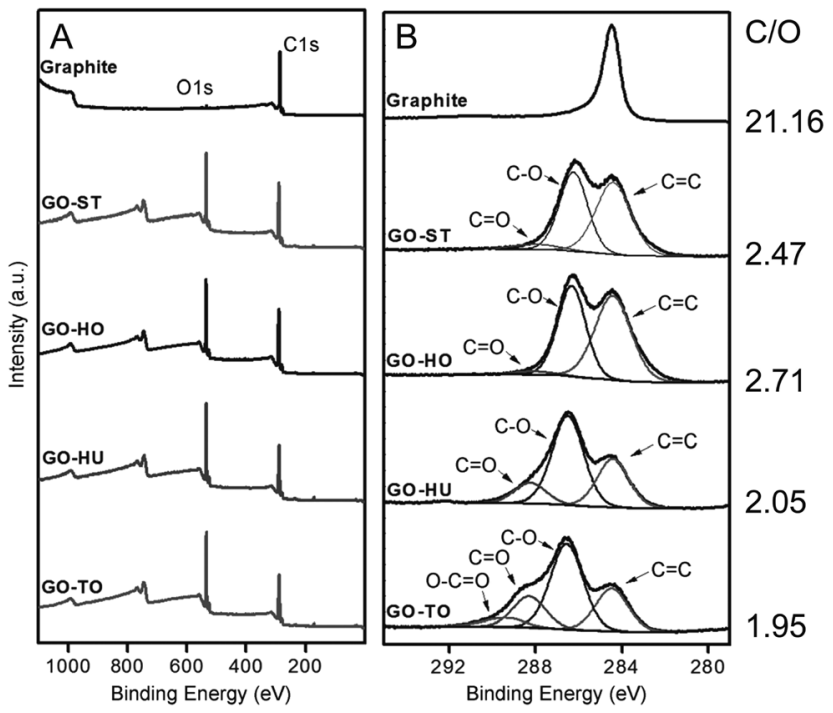

Fig. 9 X-ray photoelectron spectra of graphite, GO-ST, GO-HO, GO-HU, and GO-TO. (A) Survey and (B) C1s core-level spectra. Reproduced from ref. 25 with permission from Wiley- $\mathrm{VCH}$.

XPS deconvolution is also used to calculate the amount of individual oxygen functionalities of GOs. ${ }^{26}$ To evaluate the $\mathrm{C} / \mathrm{O}$ ratio and to compare the GO materials obtained from different synthetic methods and conditions, XPS survey scan is used to provide information about the presence of any element on the materials. Based on Fig. $9,^{25}$ the XPS survey spectra indicated obvious peaks of carbon (282-292 eV) and oxygen (529-539 eV) elements on graphite, GO-ST (Staudenmaier), GO-HO (Hofmann), GO-HU (Hummers) and GO-TO (Tour). The low atomic C/O ratios $(\sim 2)$ observed for all the GO materials indicated successful and extensive oxidative treatments. Overall, the permanganate (GO-HU and GO-TO) oxidation methods showed a higher extent of oxidation compared with the chlorate (GO-ST and GO-HO) oxidation. Elemental analysis of GO-ST and GO-HO has also indicated that sulfur and nitrogen were not detected for the two materials. However, GO-HU showed the presence of these two elements. ${ }^{24}$ More details about each method are given in Section 4.

Thermogravimetric analysis (TGA) of GO shows major weight losses between 150 and $300{ }^{\circ} \mathrm{C}$, which corresponds to $\mathrm{CO}, \mathrm{CO}_{2}$, and steam release from the most labile functional groups. Between 400 and $950{ }^{\circ} \mathrm{C}$, a slower mass loss was observed and is attributed to the removal of more stable oxygen functionalities. By TGA, GO-HU has a smaller weight loss than GO-TO. ${ }^{13} \mathrm{C}$-SS-NMR suggests that the order of overall oxidation in GO-HU is smaller than in GO-TO. The simplest measure of oxidation is the ratio between the alcohol/epoxide signal and the graphitic $\mathrm{sp}^{2}$ carbon signal. This ratio is the highest for GO-TO and lowest for GO-HU. It is also noteworthy that GO-TO appears to have more epoxide functionalities than either of the other GOs. ${ }^{27}$ The use of permanganate oxidants generates distinctive oxygen functionalities that do not arise from other sources. Specifically, the increased presence of carbonyl and carboxyl groups on GO, which is a unique phenomenon for the materials prepared through the Hummers' and Tour methods, affects directly the $\mathrm{p} K_{\mathrm{a}}$ of $\mathrm{GO}$ and the $\mathrm{pH}$ of its suspension in water. Furthermore, the oxygen functionalities are electrochemically active and have different reduction potentials. Those differences in oxygen composition give a distinct electrochemical behavior for GO prepared through the permanganate oxidant routes (Hummers and Tour) compared with those prepared using chlorate oxidants (Staudenmaier and Hofmann). ${ }^{28}$ The $\mathrm{C} / \mathrm{O}$ ratio of $\mathrm{GO}$ is an important parameter that affects directly the number of oxygen atoms attached to the carbon, through modifying its hydrophobicity, the way it interacts in colloids, its chemical reactivity, its sheet resistance $(\mathrm{M} \Omega)$, and the way it stacks over itself in the solid state (interlayers). ${ }^{4,7,29}$

\subsection{Reduced form of GO}

For tailoring materials with properties as close to pristine graphene as possible, exhaustive research has been done to remove the oxygen functional groups from GO. ${ }^{30}$ This reduction can be accomplished by means of microorganisms, ${ }^{31}$ chemicals, ${ }^{23}$ electrochemistry, ${ }^{7,32}$ heat, ${ }^{4,7} \mathrm{UV},{ }^{2,4}$ microwave irradiation ${ }^{33}$ or ion bombardments ${ }^{34}$ to get tunable properties mostly for applications in electronics. ${ }^{5,6,35}$ The key designs include the $\mathrm{C} / \mathrm{O}$ ratio of the end product (graphene-rGO), selective removal of a single type of oxygen group (hydroxyl vs carboxylic acid vs epoxy, etc.), healing of the surface defects from oxidation, and choice of green and safe conditions, as well as maintaining or improving the desired chemical and physical properties of GO (mechanical strength, conductivity, optical properties, solubility/dispersibility of nanosheets, etc.). ${ }^{36}$

After the reduction process using thermal ( $\mathrm{T}$ ) exfoliation, TEM micrographs of TrGO-ST, TrG-HO and TrG-HU display wrinkles, single-to-few layer rGO sheets which are typical of graphene, indicating an effective exfoliation of GO. Scanning electron microscopy (SEM) and transmission electron microscopy (TEM) analyses show no major differences in the morphologies between methods. ${ }^{24}$ From the Raman measurements, it can be concluded that TrGO-ST is capable of producing GO with the lowest density of defects. ${ }^{24}$ When the classical reductive agent hydrazine is used, there is larger variability in composition and properties of rGO obtained from GO prepared by various oxidation methods. The $\mathrm{C} / \mathrm{O}$ ratios for chemically reduced GO (CrGO) are in the range between $\sim 4$ and $\sim 11$. On the other hand, the ratios for thermally reduced GO (TrGO) are much higher ranging from $\sim 22$ to $\sim 50$. CrGO also contains higher amounts of nitrogen $(\mathrm{N})$ and hydrogen $(\mathrm{H})$ compared to TrGO. This originates from unintentional doping by nitrogen using hydrazine as a reducing agent. Electron spectroscopy chemical analysis of CrGO shows lower nitrogen content for CrGO-HU compared to CrGO-TO. The highest $\mathrm{C} / \mathrm{O}$ ratio was found for TrGO-HU and TrGO-TO synthesized from highly oxidized graphite oxides. GO prepared by permanganate methods contains high concentration of thermally labile ketone and carboxylic acid functionalities which easily undergo decomposition under the formation of $\mathrm{CO}_{2}$ and suppression of oxygen concentration. In comparison, the chlorate-based GO contains predominantly hydroxyl functional groups which have significantly higher thermal stability. Low surface areas were also observed on CrGO-BR and 
CrGO-HO prepared by the chlorate methods. CrGO originating from GO prepared by permanganate methods exhibits surfaces that are comparable to TrGO. The exfoliation of CrGO is significantly higher for the samples originating from GO prepared by permanganate routes compared to chlorate methods. The main exception from all trends is rGO-ST showing a lower degree of oxidation leading to a markedly lower degree of exfoliation and subsequently to higher differences in the observed properties such as surface area, stability towards oxidation and others. Interestingly, all rGO have comparable HET rates using a $\left[\mathrm{Fe}(\mathrm{CN})_{6}\right]^{-3}$ redox probe. $^{37}$

The correlations between the oxygen content and the properties of GO and rGO are important benchmarks when utilizing GO in practical applications. Electronic conductivity is one of the important properties of graphene-like materials. It is evaluated using four-point probe measurements. ${ }^{27}$ The electrical conductivity of GO is dramatically decreased when the oxygen content exceeds $25 \mathrm{wt} \%$. For a medium oxygen content (30-40 wt\%), rGO shows better conductivity than GO. By contrast, for a low oxygen content (10-20 wt\%), the conductivity of rGO is less than that of GO. Incomplete recovery of the $\mathrm{sp}^{2}$ domains and defects would inhibit the electron conductivity of rGO. As the oxygen content of the original GO increases, irreversible damage of the graphene structure occurs during the reduction and the electrical conductivity of the end-product (rGO) is affected. The surface area and electrical conductivity of a material are closely related to the capacitance. Because of its high surface area, moderate conductivity, and low oxidizing ability, rGO with 20-40 wt\% oxygen content has practical applications such as in electric double-layer capacitors, thermoconductive films, reinforcing layers of polymers, support materials of catalysts, and biosensors. ${ }^{30}$

By comparing the electrical conductivity of CrGO and CrGO treated thermally (TCrGO) prepared from both the Hummers' and Tour methods, Tour and collaborators showed that CrGOHU-based devices exhibited conductivity values of $0.05 \mathrm{~S} \mathrm{~cm}^{-1}$, whereas CrGO-TO monolayers were about twice as conductive $\left(0.1 \mathrm{~S} \mathrm{~cm}^{-1}\right)$. Since hydrazine alone in the reduction is not sufficient to achieve high conductivities of the rGO flakes, CrGO is treated thermally by annealing in $\mathrm{Ar} / \mathrm{H}_{2}$ at high temperatures. All TCrGO exhibit similar increases in conductivities up to 350$375 \mathrm{~S} \mathrm{~cm}^{-1}$ (TCrGO-HU), and $400 \mathrm{~S} \mathrm{~cm}^{-1}$ (TCrGO-TO). ${ }^{27}$ Furthermore, it was found that the type of GO precursor has a major influence on the level of doping. Due to the differences in the structures of GO-ST, GO-HO and GO-HU, GO-HU can be thermally doped in the presence of ammonia gas with the highest amount of nitrogen (7.2 at\%) and GO-ST can be doped with the lowest amount of nitrogen $(0.94 \mathrm{at} \%) .{ }^{38}$ A similar trend is also observed for sulphur-doped graphene in the presence of $\mathrm{H}_{2} \mathrm{~S}, \mathrm{SO}_{2}$ or $\mathrm{CS}_{2}$ gas. $^{39}$ This has eminent implications for any application of graphene in electrochemical devices for energy storage and sensing applications. ${ }^{24}$

\subsection{Impurities}

3.3.1 From the methods. The exact structure of GO is highly dependent on the method of preparation ${ }^{28}$ and the source of carbon (Section 4) and might include impurities ( $\mathrm{Na}, \mathrm{K}, \mathrm{Mn}, \mathrm{S}, \mathrm{N}, \mathrm{Cl}, \mathrm{P}$ ).
These impurities originate from the chemicals used for the synthesis of GO and persist also in graphene after its reduction, ${ }^{23}$ introducing new energy levels into the electronic structure, which is responsible for tuning the optical, ${ }^{40}$ electrical and catalytic properties. In general, the properties like surface area, morphology, electrical conductivity, transparency and residual oxygenation are controlled not only by the degree of oxidation, but also by the composition of oxygen functionalities and the method of reduction. ${ }^{37}$ Residues of $\mathrm{N}, \mathrm{S}$ and $\mathrm{Mn}$ in the resulting graphene play an extremely active role in the electrocatalysis of the oxygen reduction reaction. ${ }^{41,42}$ Those impurities are present abundantly in graphene prepared by the Hummers' oxidation method which applies a large amount of permanganate oxidant. More dramatic is the presence of residual $\mathrm{K}$ salts in GO. The presence of potassium salt impurities causes catastrophic reduction (carbon combustion) of GO upon fast heating or simply by friction. The self-propagating reduction from GO solids is due to its highly energetic nature, suggesting that caution needs to be adopted against a possible fire hazard. ${ }^{43} \mathrm{Cl}$ atoms introduce additional energy levels between $\mathrm{C} \pi$ and $\mathrm{C} \pi^{*}$. Chlorine-doped impurities impart to graphene quantum dots multicolour emitting properties when simply exposed to an excitation wavelength between 300 and $600 \mathrm{~nm}$. These multicolour Cl-GQDs have applications in bioimaging and optoelectronic devices. ${ }^{40}$

Heating GO under a controlled atmosphere liberates not only simple molecules $\left(\mathrm{CO}_{2}, \mathrm{CO}, \mathrm{H}_{2} \mathrm{O}\right)$ but also many volatile organic compounds (VOCs) and radicals that recombine to form more complex organic molecules. These emissions have an impact on the toxicity of the products of graphene on a larger scale and they are analysed and quantified by gas chromatography (GC) techniques coupled with a mass spectrometer (MS). The main differences in the composition of gaseous products can be seen for the GO-BR. The Brodie oxidation procedure is based on the oxidation of graphite by potassium chlorate in fuming nitric acid. In this case no significant amount of sulphur was detected in GO. All other methods (Hummers, Hofmann, and Staudenmaier, Tour), which also use sulfuric acid in addition to $\mathrm{HNO}_{3}$, led to GO containing S, mainly in the form of sulfuric acid esters, which decompose into $\mathrm{SO}_{2}, \mathrm{CS}_{2}$ and some organosulfur compounds such as thiophene, benzothiophene, and dibenzothiophene. Chlorine present in the GO is evolved in the form of chlorobenzene and nitrogen in the form of nitrile groups attached to hydrocarbons such as 2-propenenitrile and benzonitrile. Nitrogen originates from the nitrogen moieties introduced by the presence of nitric acid (or sodium nitrate in the case of the Hummers' method). ${ }^{26}$

3.3.2 From the metallic contaminants. Graphene prepared from graphite does not require the usage of a metal catalyst, and for this reason, the presence of metallic impurities has not been considered an issue. ${ }^{44}$ They were found in levels widely considered to be trace and negligible but still enough to exert a significant influence on the final graphene. ${ }^{44-47}$ But it has been demonstrated thoroughly that synthetic routes contaminate graphene materials with a whole spectrum of metallic elements, present in parent natural and synthetic graphite. The graphite particle still contains some mineral impurities, which are 
"intercalated" or stacked between adjacent graphene layers. These impurities persist in the samples of GO and rGO after the oxidative and reductive treatments, ${ }^{45}$ and they can only be partially removed by chemical or thermal treatments. Based on the results of induced coupled plasma spectroscopy coupled with a mass spectrometer (ICP-MS), it is clear that the metallic impurities, in particular $\mathrm{Fe}$ and $\mathrm{Ni}$, are still present after the procedures followed to fabricate graphene. They may alter properties such as electrochemical behavior, redox potential of biomarkers and adsorptivity. Fe and Ni display important catalytic effects with possible implications in toxicology. ${ }^{46}$ They are responsible for some "noble metal catalyst free" synthetic reactions such as the oxygen reduction reaction (ORR) ${ }^{42}$ and the hydrogen evolution reaction (HER). ${ }^{47}$

Metallic contamination with elements other than the heteroatoms associated with the oxidation methods is found in GO. Elements such as $\mathrm{As}, \mathrm{Co}, \mathrm{Cr}, \mathrm{Cs}, \mathrm{Cu}, \mathrm{Fe}, \mathrm{Sb}$, and $\mathrm{Sc}$ are also introduced into the GO-HU sample after the oxidative treatment. The origin of most of these elements is likely the chemical reagents. For example, ICP and optical emission spectrometry (OES) data of $\mathrm{KMnO}_{4}$ and $\mathrm{NaNO}_{3}$ showed the presence of eight metallic elements ( $\mathrm{Ca}, \mathrm{Co}, \mathrm{Cr}, \mathrm{Cu}, \mathrm{Fe}, \mathrm{Ni}, \mathrm{Pb}$, and $\mathrm{Zn}$ ). With regard to the oxidative methods, GO-ST shows only slight changes in the metallic contents when compared to the precursor graphite. However, substantial decreases in the metallic contents were observed with GO-HU from natural graphite. Atomic absorption spectroscopy (AA) of GO-ST revealed 11 different trace metallic elements similar to those in GO-HU, with a total of 15 metallic elements. The decreased amounts of impurities in GO-ST compared with GO-HO can be attributed to the use of fuming nitric acid much more corrosive and effective at removing metal in the Staudenmaier method than the concentrated nitric acid used in the Hofmann method. ${ }^{44}$ Other treatments for reducing the amount of metallic impurities are based on (i) soaking and refluxing in an aqua-regia mixture; (ii) sonication in a mixture of hydrogen peroxide and hydrochloric acid; and (iii) thermal treatment in $\mathrm{a}_{2}$ atmosphere. ${ }^{45}$

\subsection{Stacking}

3.4.1 Solid state. In GO, atoms of carbon (C), oxygen (O) and hydrogen $(\mathrm{H})$ are regrouped under organized monolayers having a thickness of around $1 \mathrm{~nm}$ (Fig. 10), ${ }^{48}$ depending on the level of hydration and the method used to measure its thickness. GO particles can have the shape of a flake, a sheet, a quantum dot (sphere), a platelet or a ribbon with dimensions ranging from the nanometer $(\sim 4 \mathrm{~nm})$ to the millimeter $(\sim 3 \mathrm{~mm}) .^{5-7}$

Very similar in structure and chemical composition, graphite oxide, its multilamellar-parent compound, is composed of many layers of GO stacked one over the other with a sheet interlayer distance of approximately 0.7-1.0 nm. Oxidation methods showed a variation in the spacing between the graphene layers. The GO prepared using the Tour method (GO-TO) exhibits the largest distance between graphene layers $(0.95 \mathrm{~nm}),{ }^{27}$ followed by the Hummers' method (GO-HU) with a value of $0.8133 \mathrm{~nm}$, and the Hofmann method (GO-HU) at $0.7226 \mathrm{~nm}$. The smallest spacing was found in the Staudenmaier method (GO-ST) at
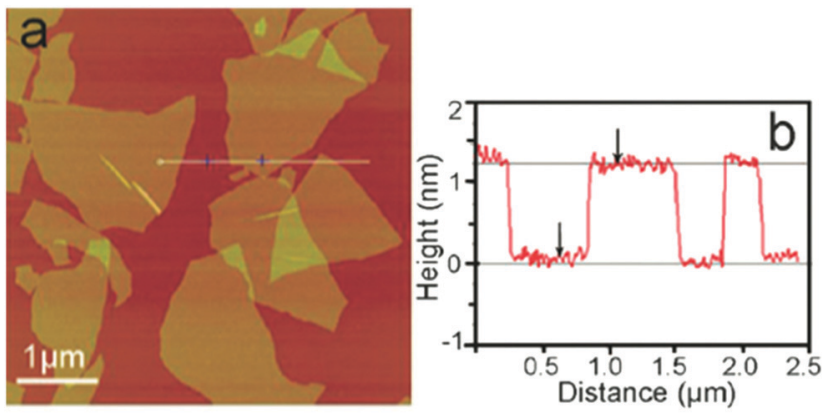

Fig. 10 (a) A tapping mode AFM image of graphene oxide (GO) sheets on a mica surface, (b) the height profile of the AFM image. Reproduced from ref. 48 with permission from the Royal Society of Chemistry.

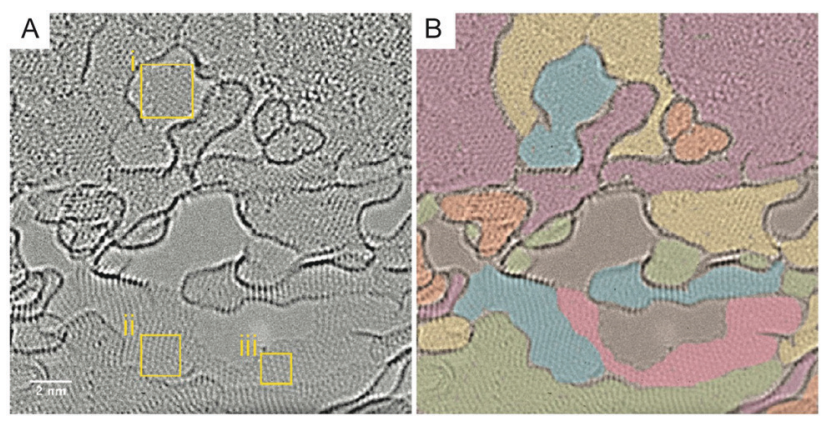

Fig. 11 (A) High-resolution image of mono- and few-layer graphene oxide at $700{ }^{\circ} \mathrm{C}$ imaged with an in situ TEM heating holder. (B) Overlaid color represents layers of the material, showing turbostatic stacking and thicker and thinner regions. Reprinted with permission from (ACS Nano, 2016, 10, 7515-7522). Copyright (2016) American Chemical Society.

$0.7084 \mathrm{~nm} .^{24}$ Fig. 11 shows how GO sheets stack one over the other to form thicker and thinner regions via hydrogen bonding mostly. ${ }^{49}$ Water molecules, reagents, inorganics, metals, ions ${ }^{34,50}$ and polar solvents can bind tightly to the structure of GO increasing the interlayer as well. ${ }^{51}$ When the stacking involves two (2) sheets of GO, the system is called a bi-layer and when three (3) sheets are involved, it's called a tri-layer. When the stacking involves four (4) to ten (10) sheets, the system is usually called few-layer; above ten (10) layers, it is referred to as a multilayer, multilamellar or graphitic system. ${ }^{7}$

3.4.2 Spacers and cross-linkers. Graphene oxide (GO) is an emerging star for nano-building ${ }^{52}$ and shows great potential in membrane technology for water remediation ${ }^{53,54}$ and molecular separation. ${ }^{55}$ Similar to carbon nanotubes, graphene oxide sheets are assembled into a paper-like material under a directional flow, yielding, after drying, a free-standing graphene oxide paper with thicknesses ranging from 1 to $30 \mu \mathrm{m} .{ }^{56}$ Since their first synthesis by vacuum filtration of colloidal dispersions of graphene oxide using membrane filters, many other methods for the synthesis of graphene-based papers from GO sheets have been developed. For example, by self-assembly of GO membranes by evaporating the hydrosol, or by spin-coating to fabricate few nanometer thin GO foils for radio frequency resonators. Cross-linking the GO sheets already in dispersion and subsequently evaporating the solvent to make cross-linked GO papers represent an alternative approach. 
The mechanical properties of GO papers can be further enhanced by modification with a small amount of divalent alkaline earth metal ions ${ }^{50}$ bonded to the functional groups on GO sheets. The divalent ion serves as a cross-linking element between two neighboring carboxyl groups of the GO sheets and increases their mechanical properties. Magnesium and calcium are ideal choice for such purposes. The typical carbon/oxygen ratio of such papers ranges from 1.2 to $2.7 .{ }^{34}$ In addition, GO can also form hybrids when mixed with other materials acting as spacers to prevent GO restacking. For this purpose, carbon nanotubes, ${ }^{57,58}$ metal and metal oxide particles, ${ }^{59}$ reinforcement polymers, and small organic molecules covalently or non-covalently bonded b $^{2,3,5-8,60}$ are widely studied to make porous graphene materials for supercapacitors. Polyaniline is mostly studied among conducting polymers. Different metal oxides, such as $\mathrm{MnO}_{2}, \mathrm{Fe}_{3} \mathrm{O}_{4}, \mathrm{NiO}$, and $\mathrm{RuO}_{2}$, have also been reported for use with graphene. Similarly, carbon materials, such as activated carbon or carbon nanotubes, nanosized and functionalized carbon black particles attached onto the surface of the graphene sheets, have also been used for serving as spacers to separate and support the neighboring sheets. ${ }^{61}$

\subsection{Interactions}

3.5.1 $\mathbf{p H}$ and $\mathbf{p} K_{\mathrm{a}}$. A GO sheet can be recognized as a single layer graphite sheet with various hydrophilic oxygenated functional groups. The hydroxyl and epoxide groups are mainly on the basal planes and ionizable groups are mostly at the edges of GO sheets. The electrostatic repulsion between GO sheets, resulting from their ionized carboxyl and phenol groups, prevented their aggregation in aqueous medium. ${ }^{51}$ The edge-to-area ratio of a GO sheet increases with the decrease of its lateral dimension. Thus, in the aqueous media, the smaller GO sheets should have higher solubility than their larger counterparts because of higher densities of ionized groups. Furthermore, the solubility of GO sheets in water also decreases with the decrease of the $\mathrm{pH}$ value of their dispersion. This is mainly because the repulsion

$$
\mathrm{GOH} \stackrel{K_{\mathrm{A}}}{\rightleftharpoons} \mathrm{GO}^{-}+\mathrm{H}^{+}
$$

Scheme 4 Dissociation of GO in water. Reproduced from ref. 65 with permission from the Royal Society of Chemistry. force between GO sheets is weakened by the protonation of their carboxyl and phenol groups. Therefore, the GO dispersion in water can be acidified to suitable $\mathrm{pH}$ values $(\mathrm{pH}=4)$ for selectively precipitating large GO sheets. ${ }^{62}$ In organic polar solvents such as ethanol, size fractionation of GO sheets can be achieved by selective natural deposition. When GO is dispersed in ethanol and allowed to stand for a while, the larger sized GO sheets are deposited first, and the smaller sized sheets remain dispersed in the solvent. It was found that GO sheets prepared by this method were highly homogeneous in size. ${ }^{63}$ In solution, GO sheets normally carry a negative charge $(\mathrm{pH}>4)$ and have three distinct $\mathrm{p} K_{\mathrm{a}}$ values of 4.3 , 6.6 and 9.0 due to the dissociation of carboxylic, phenolic and hydroxyl groups (Scheme 4). ${ }^{64,65}$

3.5.2 Electric double-layer. Surface-charged GO sheets can be described as a flat slab with two faces using the theory of the electric double layer (EDL). Obviously, $\mathrm{pH}$ conditions and the presence of an electrolyte determine the surface charge density, potential, and interaction between GO sheets, because the static interaction between GO sheets will be attenuated at high electrolyte concentration. In the presence of an electric field, GO will orientate and migrate. The interaction between GO and the field will be different for sheets with different sizes, thicknesses, and charges. These effects are the key factors that determine the separation of GO fractions during electrophoretic migration. The zeta $(\zeta)^{51}$ potential of the fragments, sheets, or particles is also an important factor for characterizing the dispersion stability and hydrodynamic properties of the GO suspension. Zeta potential is the potential difference between the dispersion medium and the stationary layer of fluid attached to the dispersed particle. Moreover, the absolute value of the $\zeta$ potential can be used to quantify the Coulomb repulsion between GO sheets, and a value of $30 \mathrm{mV}$ is the threshold above which colloids are stable and not prone to aggregation. ${ }^{65}$

3.5.3 Colloids. During the sonication-assisted exfoliation process of $\mathrm{GO},{ }^{51,66}$ and $\mathrm{rGO},{ }^{29}$ the layered crystal is sonicated in a solvent, resulting in exfoliation and nanosheet formation. In "good" solvents, those with appropriate surface energy, the exfoliated nanosheets are stabilized against reaggregation. Otherwise, for "bad" solvents reaggregation and sedimentation will occur (Fig. 12). ${ }^{66}$ This mechanism also describes the

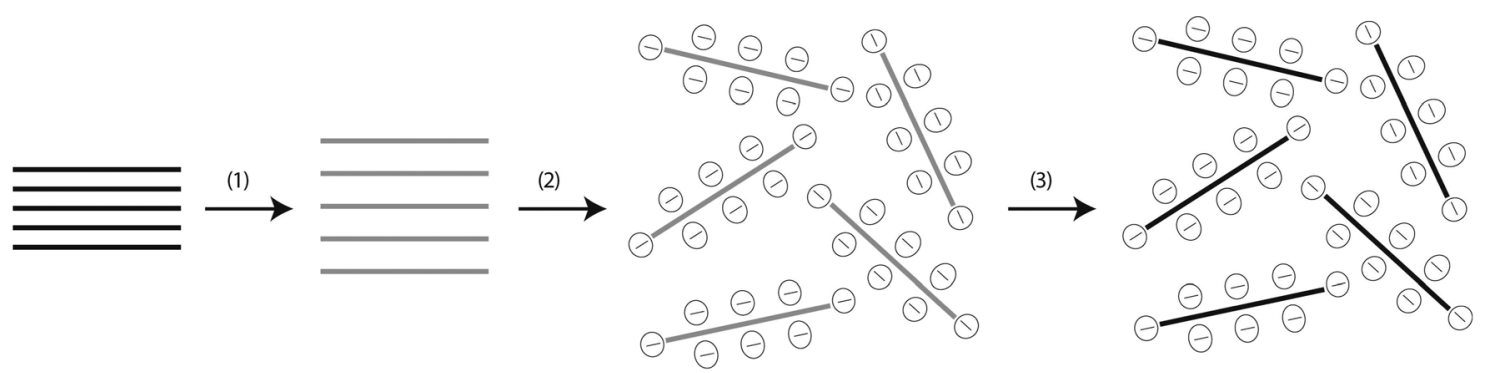

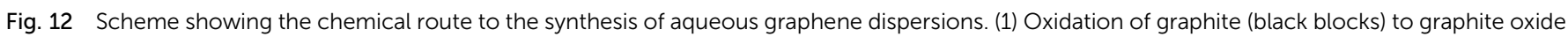

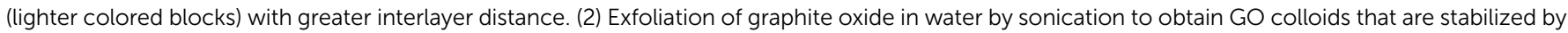

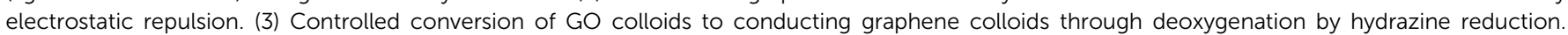
Reproduced from ref. 51 with permission from the NPG. http://creativecommons.org/licenses/by/4.0/. 


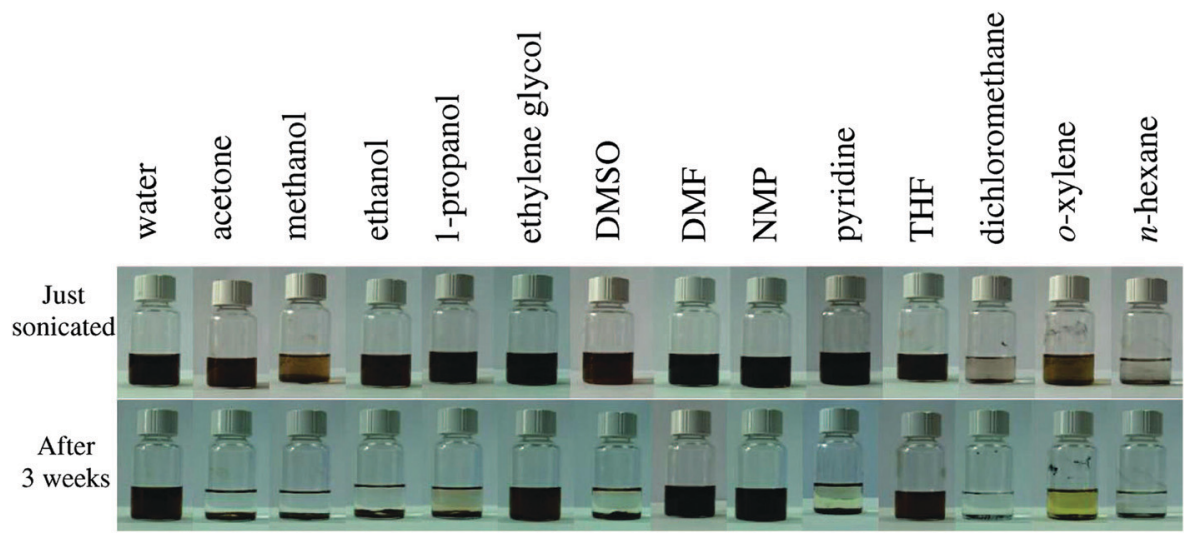

Fig. 13 Digital pictures of as-prepared graphite oxide dispersed in water and 13 organic solvents through bath ultrasonication (1 h). Top: Dispersions immediately after sonication. Bottom: Dispersions 3 weeks after sonication. The yellow color of the 0 -xylene sample is due to the solvent itself. Reprinted with permission from (Langmuir, 2008, 24, 10560-10564). Copyright (2008) American Chemical Society.

dispersion of GO and rGO in polar solvents (Fig. 13), such as water, and in organic solvents, such as polypropylene carbonate, ${ }^{67}$ ethylene glycol, DMF (dimethyl formamide), NMP ( $N$-methyl pyrrolidinone) and THF (tetrahydrofuran). ${ }^{68}$ As demonstrated in many colloid experiments, the colloidal stability of an electrostatically stabilized dispersion is strongly dependent on $\mathrm{pH}$, the electrolyte concentration, and the content of dispersed particles. It is well known that exfoliated graphite oxide (or rGO) can form well-dispersed aqueous colloids and is negatively charged when dispersed in water, apparently as a result of ionization of the carboxylic acid and phenolic hydroxyl groups that are known to exist on the sheets. This suggests that the formation of stable $\mathrm{GO} / \mathrm{rGO}$ colloids should be attributed to electrostatic repulsion, rather than just the hydrophilicity of GO as presumed. ${ }^{29,53,66}$

\subsection{GO analogs with high degree of functionalization}

Stoichiometric derivatives of graphene with other chemistries having a high degree of functionalization and a well-defined structure are of great interest to the 2D materials research. While GO contains a non-stoichiometric number of oxygenated groups with mixed compositions on its structure, graphene derivatives with exclusive existence of carboxyl or hydroxyl (graphol) are also reported (see Sections 3.6.1 and 3.6.2). Furthermore, graphene can covalently interact with other elements such as hydrogen (graphane), fluorine (fluorographene), sulfur (thiographene) and nitrogen (amino- and cyanographene), allowing their further transformation and device implementation. ${ }^{69}$

3.6.1 Carboxy graphene. Carboxylic acid functions on the graphene's structure is of pivotal importance for several applications including electrochemical sensing ${ }^{70}$ and transparent conductive films assembled with inorganics. ${ }^{71} \mathrm{COOH}$ functions are highly hydrophilic and improve the dispersibility of graphene in water and the long-term stability of the colloidal solutions. These groups are typically located on the edges and defect sites of graphene, since these carbon atoms are significantly more reactive. ${ }^{69}$ They are used for controlling GO separation and transport properties and to make covalent bonds with larger molecules via functionalization..$^{5-7} \mathrm{COOH}$ also improves the sorption capacity toward heavy metals for environmental remediation applications. ${ }^{30}$ Furthermore, $\mathrm{COOH}$ on GO improves its mechanical properties and contains electronegative atoms $(\mathrm{O})$ which strongly affects its electronic structure. ${ }^{69}$ Ways to increase the number of $\mathrm{COOH}$ on GO depends on the oxidation conditions of the bulk graphite (see Section 4.1$)^{72,73}$ or using more than one oxidizing steps in sequence. ${ }^{74}$ The Diels-Alder (DA) reaction enables the addition of $\mathrm{COOH}$ after hydrolysis of the adduct formed with maleic anhydride. $^{21}$ The Claisen rearrangement of allylic alcohol groups found on GO reacted with a vinyl transfer agent allows newly grafted $\mathrm{COOH}$ moieties after hydrolysis of the intermediate amide. $^{22}$ Carboxy graphene with a stoichiometric amount of $\mathrm{COOH}$ groups can be obtained directly from graphene using UV photo-irradiation (see Section 4.3). Very promising and selective graphene nitrile is synthesized by the reaction of $\mathrm{NaCN}$ with fluorographene, which leads to carboxy graphene after hydrolysis. ${ }^{75}$ Besides those approaches, mechanochemical direct introduction of carboxylic acid functionalities on the edge of graphene was also reported using high-energy ball milling of graphite with dry ice. ${ }^{76}$

3.6.2 Hydroxy graphene. The hydroboration of graphene oxide is one of the well-established synthetic chemistry routes that can be applied to create a monovalent hydroxy derivative of graphene (graphol). ${ }^{77}$ Hydroboration is an addition reaction on a carbon-carbon double bond that results in an organoboron moiety. The $\mathrm{C}-\mathrm{BH}_{2}$ moiety can be subsequently converted to hydroxy groups $(\mathrm{C}-\mathrm{OH})$ by the reaction with hydrogen peroxide $\left(\mathrm{H}_{2} \mathrm{O}_{2}\right)$ or to a C-H bond if reacted with an acid. In addition, all ketone groups react with borane and are converted to hydroxy groups at the same time. The resulting materials have a stoichiometry of $\mathrm{C}_{1} \mathrm{O}_{0.78} \mathrm{H}_{0.75}$.

A simple, high-yielding, one-step strategy for the preparation of graphol consists of reacting fluorographite ${ }^{78}$ or iodographene ${ }^{79}$ with sodium hydroxide under solvothermal conditions. Since the hydroxyl substituents are sufficiently hydrophilic, the solvent water molecules can easily enter into the layers, resulting in the complete exfoliation of the materials. Ball milling, in the solid state, of solid $\mathrm{KOH}$ powder and graphite flakes gives hydroxyl-functionalized 
graphene in one step. The resultant graphol shows good hydrophilicity, electroactivity and biocompatibility with human RPE cells. ${ }^{80}$ Hydroxyl in graphene is an important chemical group that influences the tribological properties of materials by creating $\mathrm{H}$ bonds in the layered lattice structure, hence, by modifying intersheet shear resistance. Methods to control its load are crucial to develop effective graphene-based lubricants. ${ }^{81}$

\section{Synthetic routes for the preparation of bulk graphite/graphene oxide}

\subsection{Oxidative exfoliation of graphite}

\subsubsection{Chemical methods}

4.1.1.1 Chlorate

Brodie method. The preparation of multilayered graphene oxide (graphite oxide) dates back to 1859 when Benjamin Brodie $^{82}$ treated graphite with strong oxidizers, for the first time, with the goal to elucidate its structure. In the historical experiment, Brodie heated lamellar graphite (with distinction to amorphous) in a mixture of potassium chlorate $\left(\mathrm{KClO}_{3}\right)$ and fuming nitric acid $\left(\mathrm{HNO}_{3}\right)$ at $60{ }^{\circ} \mathrm{C}$ for a few days $(3-4 \mathrm{~d})$. The product he obtained (GO-BR) after multiple oxidative treatments (four to seven), washing and drying process showed a composition of carbon, hydrogen and oxygen and its averaged general formula was estimated to be $\mathrm{C}_{11} \mathrm{H}_{4} \mathrm{O}_{5}$ (C/O: 2.2). Also, Brodie observed that the product was soluble in pure or basic water, while it had a tendency to flocculate in more acidic media. Furthermore, the material had a feeble acid reaction when placed upon litmus paper. For this reason, Brodie coined the term "graphic acid" (GA) to describe his new discovery (Fig. 14).

Staudenmaier, Hofmann methods. Almost forty years later, L. Staudenmaier ${ }^{83}$ ameliorated Brodie's procedure by modifying the way potassium chlorate was added to the reaction mixture and by adding sulfuric acid portionwise during the course of the reaction. More specifically, $\mathrm{KClO}_{3}$ was added in small doses to eliminate the danger associated with the evolution of explosive by-products and heat. Also, $\mathrm{H}_{2} \mathrm{SO}_{4}$ was added to increase the acidity of the mixture which drove the oxidation of graphite and shortened the reaction time as well. More importantly, at lower $\mathrm{pH}$, a smaller amount of fuming nitric acid was needed for the oxidation, though, avoiding large emission of toxic gases

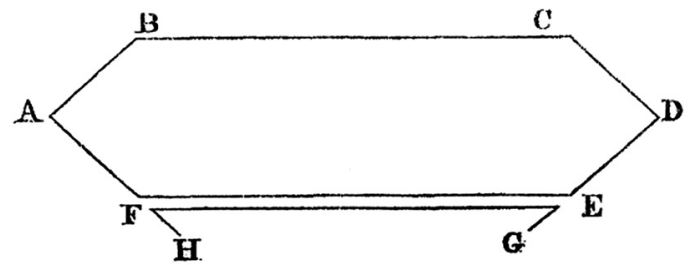

Fig. 14 Crystal of graphite oxide as observed under a microscope and described by B. C. Brodie (1859). From Philosophical Transactions of the Royal Society of London by Evelyn, John; et al. Reproduced with permission of W. Bowyer and J. Nichols for Lockyer Davis, printer to the Royal Society in the format republished in a journal/magazine via Copyright Clearance Center.
$\left(\mathrm{NO}_{2}, \mathrm{~N}_{2} \mathrm{O}_{4}\right)$ and the handling of a large quantity of corrosive and hazardous materials, which made the overall process safer. This way, he obtained materials (GO-ST) with similar properties to GO-BR. In 1937, Hofmann also developed a safe alternative using $\mathrm{KClO}_{3}$ and non-fuming nitric acid alone to prepare GO-HO with a lower level of oxidation $(\mathrm{C} / \mathrm{O}>2.5) \cdot{ }^{84} \mathrm{KClO}_{3}$ is a powerful oxidant and is used as an in situ source of molecule oxygen while nitric acid is known to react strongly with aromatic carbon surfaces, including carbon nanotubes. ${ }^{7}$ Although the conditions using $\mathrm{KClO}_{3}$ and $\mathrm{HNO}_{3}$ were developed at the beginning of the discovery of GO, they were among the most powerful oxidative methods known in that time and are still used today for the preparation of GO on the preparative scale.

\subsubsection{Permanganate}

Hummers' method. The most important method in the field of oxidative chemical exfoliation of graphite was developed by Hummers and Offeman, ${ }^{85}$ almost one hundred years after the initial discovery of Brodie. Hummers and Offeman developed the fastest and safest conventional method of producing GO-HU with a $\mathrm{C} / \mathrm{O}$ ratio (2.25) very similar to that of GO-BR (2.2). Their method was based on the action of an excess of potassium permanganate, sulfuric acid and a small amount of sodium nitrate over graphite. Their method was much safer than the chlorate-based methods because the evolution of explosive $\mathrm{ClO}_{2}$ was avoided. Also, the excess of $\mathrm{KMnO}_{4}$ was conveniently neutralized with a diluted solution of $\mathrm{H}_{2} \mathrm{O}_{2}$ to form non-toxic manganese sulfate salts and gaseous oxygen easily removed during the purification steps. Nevertheless, nitric acid was generated in situ upon acidification of sodium nitrate and some toxic gases $\left(\mathrm{NO}_{2}, \mathrm{~N}_{2} \mathrm{O}_{4}\right)$ were still produced. So far, the materials produced by the Brodie, Staudenmaier or Hummers' method have shown more defects (holes, wrinkles, irregular shape, presence of contaminants, etc.) than those produced directly from micromechanical cleavage of graphite. ${ }^{7}$ Hummers' method remains a key point of interest because it is an easy reaction suitable for producing large quantities of graphite oxide (MlGO) that can be fully delaminated into GO using liquid exfoliation techniques (Section 5). However, it yields GO with traces of sulfur (up to $6 \%)^{19}$ and nitrogen likely due to the covalently bonded sulfates and nitrates or adsorbed sulfuric and nitric acids. ${ }^{24}$

Tour's method. Noteworthily, an improved procedure for the preparation of GO with a high level of oxidation using a modified Hummers' method was developed in $2010 .^{27}$ Phosphoric acid $\left(\mathrm{H}_{3} \mathrm{PO}_{4}\right)$, a dispersive and etching agent, was used instead of sodium nitrate to assist the oxidative chemical exfoliation of graphite and to produce GO in higher yield. The GO prepared using the Tour method (GO-TO) has a higher level of oxidation and has a more regular structure with fewer defects in the basal plane compared to GO-BR, GO-ST and GO-HU. The more regular structure is explained by the formation of five-membered cyclic phosphate groups between the phosphoric acid and two vicinal diols formed on the graphite basal plane. This one pot approach is very popular and is often recognized as the "fourth" principal method to prepare GO after Brodie, 


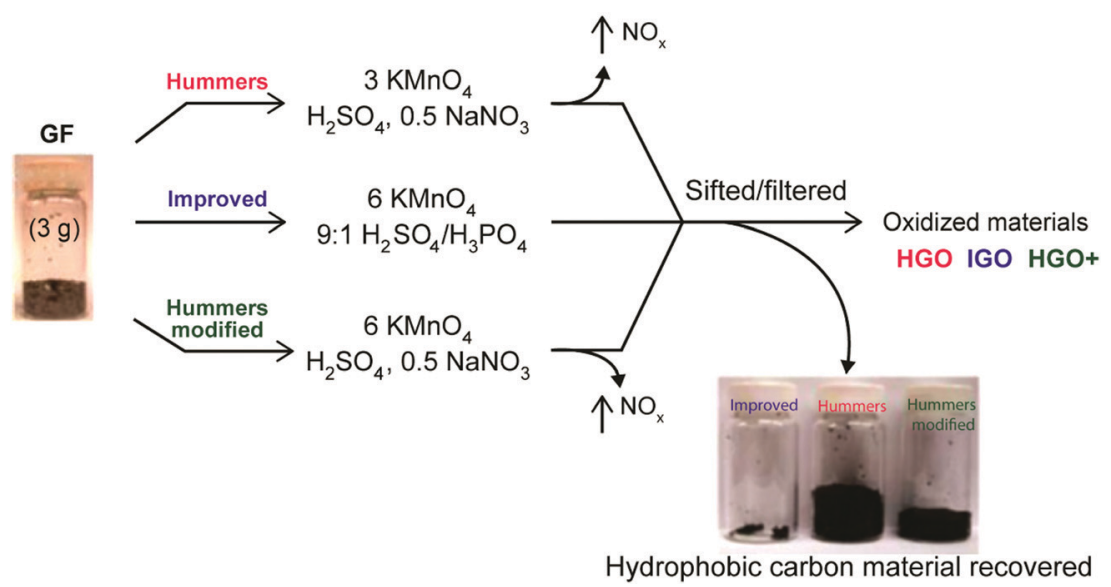

Fig. 15 Representation of the procedures followed starting with graphite flakes (GF). Under-oxidized hydrophobic carbon material recovered during the purification of IGO, $\mathrm{HGO}$, and $\mathrm{HGO}^{+}$. The increased efficiency of the IGO method is indicated by the very small amount of under-oxidized material produced. Reprinted with permission from (ACS Nano, 2010, 4, 4806-4814). Copyright (2010) American Chemical Society.

Staudenmaier and Hummers (Fig. 15). ${ }^{1-3}$ A detailed study of the mechanism of the intercalation shows how sulfuric acid and potassium permanganate intercalate into the graphite's intergalleries (Fig. 16). ${ }^{86-88}$

Mechanism of the oxidative chemical exfoliation with $\mathrm{KMnO}_{4}$. Despite recent progress in GO chemistry and structure, the mechanism of its formation gained less attention and is still not fully understood. Tour has suggested that the formation of GO from Gr proceeds in three distinct and independent steps: ${ }^{86}$

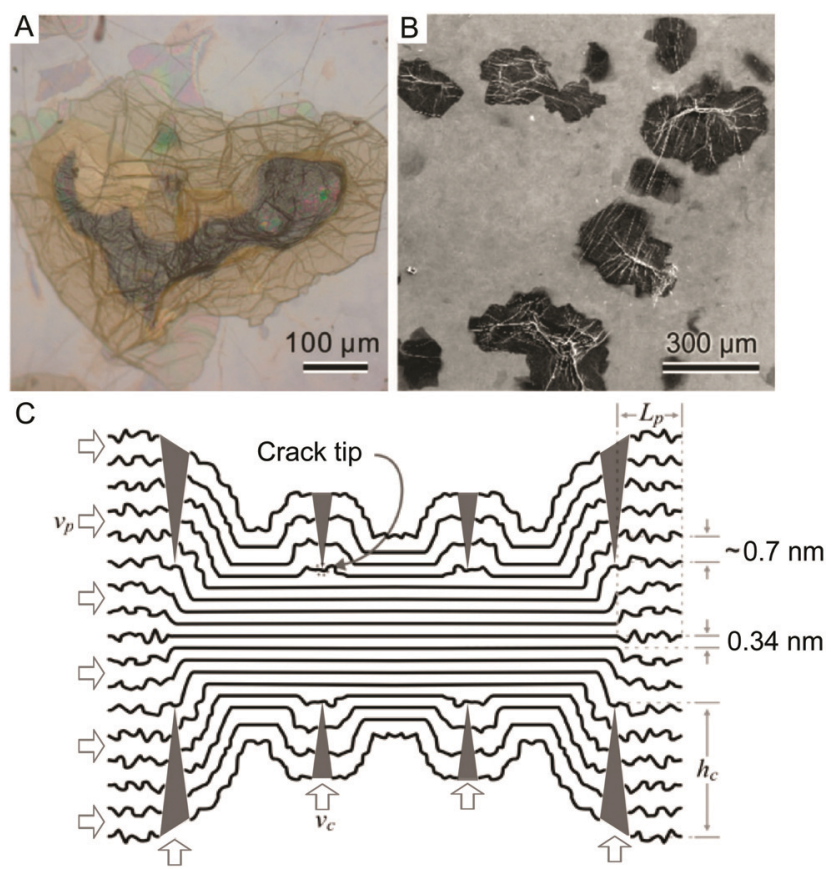

Fig. 16 (A) Optical microscopy and (B) SEM images of graphite particles oxidized after $6 \mathrm{~h}$. Schematic (C) of crack propagation and penetration of oxidizing solution during the oxidation of a graphite particle immersed in an oxidizing agent. Reprinted (adapted) with permission from (ACS Nano, 2011, 5, 4073-4083). Copyright (2011) American Chemical Society.
The first step is the conversion of Gr to the sulfuric acidgraphite intercalation compound (GIC) which made the graphite more reactive to oxidation without any important change in its structure. The second step is the conversion of the GIC into the oxidized form of graphite (OGr) defined as "pristine graphite oxide" (PGO). PGO formation is a slow diffusion-controlled process and depends on the rate of replacement of the acid intercalate by the oxidant. The oxidation would be initiated on the active positions of the graphite sheets, i.e., on the edges and imperfect regions, then on the basal plane under strong oxidation conditions (Fig. 16). ${ }^{88}$ Most likely, in this step, the reduced form of the oxidizing agent remains in the interlayer galleries and is not removed until PGO exfoliates completely upon exposure to water (step 3). In the third step, PGO is converted to GO by the reaction of PGO with water. To exfoliate PGO, the hydration by water should overcome electrostatic attraction within the intercalated compound. Also, under acidic conditions, water should hydrolyze covalent sulfate and ester groups that possibly crosslink two neighboring GO layers stabilizing PGO structure against its exfoliation into single layer GO. Furthermore, hydration is driven by hydrogen bonding and by electrostatic interaction between GO and water. This results in the formation of a colloidal suspension of GO monolayers in water.

Others have proposed also a fourth hidden step based on the oxidation of $\mathrm{GO}$ by $\mathrm{MnO}_{4}{ }^{-}$ions after the addition of water. ${ }^{89}$ Although potassium permanganate is a common oxidant, the active chemical species responsible for the conventional step of the main oxidation (step 2) of the Hummers' reaction in sulfuric acid are $\mathrm{MnO}_{3}{ }^{+}$(Scheme 5a) and $\mathrm{Mn}_{2} \mathrm{O}_{7}$ (Scheme 5b). ${ }^{86-88}$ Manganese heptoxide $\left(\mathrm{Mn}_{2} \mathrm{O}_{7}\right)$ is a dark oil formed upon contact of $\mathrm{KMnO}_{4}$ with $\mathrm{H}_{2} \mathrm{SO}_{4}$ and is explosive. It can detonate in the presence of organic molecules or upon heating to more than $55{ }^{\circ} \mathrm{C}^{7}$ It is more likely, however in step 2, that $\mathrm{Mn}(\mathrm{vII})-\mathrm{H}_{2} \mathrm{SO}_{4}$ exists in the form of planar permanganyl $\left(\mathrm{MnO}_{3}{ }^{+}\right)$cations bonded with sulfate ions and hydrogen sulfate in the form of $\left(\mathrm{MnO}_{3}\right)_{2} \mathrm{SO}_{4}$ (Scheme 5c) or $\mathrm{MnO}_{3} \mathrm{HSO}_{4}$ (Scheme $5 \mathrm{~d}$ ). ${ }^{87}$ In the reaction mixture, 


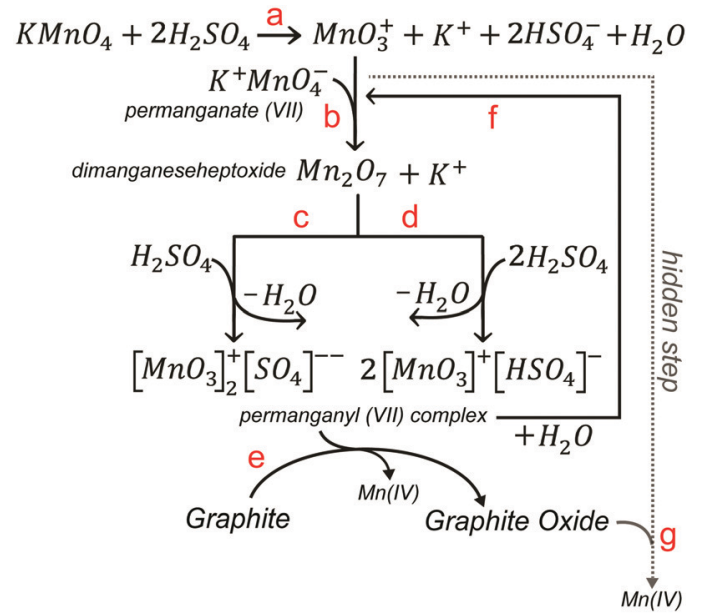

Scheme 5 (a) Formation of $\mathrm{MnO}_{3}{ }^{+}$, (b) dimanganeseheptoxide, and planar permanganyl(VII) cation bonded with (c) sulfate ion or (d) hydrogen sulfate upon the reaction of $\mathrm{KMnO}_{4}$ with $\mathrm{H}_{2} \mathrm{SO}_{4}$. (e) Formation of graphite oxide and $\mathrm{Mn}$ (IV) ion from the reaction of graphite with $\mathrm{Mn}(\mathrm{VII})$ species, (f) reversible formation of $\mathrm{MnO}_{4}{ }^{-}$upon the reaction of $\mathrm{Mn}(\mathrm{VII})$ with water and $(\mathrm{g})$ reaction of permanganate $\mathrm{Mn}(\mathrm{VII})$ ions with graphite oxide (hidden step 4).

the permanganyl $\mathrm{Mn}$ (VII) are reduced to $\mathrm{Mn}$ (Iv) ions and graphite is oxidized to graphite oxide (Scheme 5e). In the presence of sulfuric acid, $\mathrm{Mn}$ (Iv) ions react further to produce soluble manganese sulfate salts (not shown). After the addition of water (step 3), manganese (VII) oxo species mostly exist as permanganate $\left(\mathrm{MnO}_{4}{ }^{-}\right)$under the acidic aqueous conditions (Scheme 5f). Until their destruction with $\mathrm{H}_{2} \mathrm{O}_{2}, \mathrm{MnO}_{4}{ }^{-}$ions have a high oxidizing potential and coexist with GO, which further increase its oxidized domains (Schemes $5 g$ and 6).

Specific advancements into the Hummers' method. Nitrate-free: One of the disadvantages of the addition of sodium nitrate in the Hummers' method is that it can enhance oxidation on the basal planes of the graphitic sheets, which caused enormous strain resulting in rupture and peeling off. ${ }^{90}$ For this reason, it is advantageous to opt for a process based on the complete removal of $\mathrm{NaNO}_{3}{ }^{91-93}$ or by substituting it with $\mathrm{K}_{2} \mathrm{~S}_{2} \mathrm{O}_{8},{ }^{94,95} \mathrm{H}_{3} \mathrm{PO}_{4}{ }^{27}$ or $\mathrm{K}_{2} \mathrm{FeO}_{4} \cdot{ }^{96}$ The elimination of nitrate is more environmentally friendly as it stops completely the production of toxic gases such as $\mathrm{NO}_{2}$ and $\mathrm{N}_{2} \mathrm{O}_{4}$. Furthermore, it prevents the uptake of nitrogen contamination in the structure of GO. It has been shown recently that the exclusion of $\mathrm{NaNO}_{3}$ does not affect the yield of the overall reaction and GO with nearly the same properties to that of conventional methods is obtained. ${ }^{91}$ Nitrate-free approaches are generally milder than the original Hummers' method and a careful control of the amount of water and the temperature of the reaction leads to GO with either hydroxyl and epoxide-rich GO or more carbonyl-rich domains (Fig. 17), respectively. ${ }^{72}$

Two-step: First, graphite flakes may be pre-oxidized using a mixture of sulfuric acid, phosphorus pentoxide and persufate ions $\left(\mathrm{S}_{2} \mathrm{O}_{8}{ }^{2-}\right)$ to yield suspensions of individual graphite sulfate sheets $\left(\mathrm{C}_{24}{ }^{+} \mathrm{HSO}_{4}{ }^{-}\right)$very slightly oxidized. After this oxidative pretreatment, the material can be oxidized further in a second step using a nitrate-free version of the Hummers' method. This 2-step approach is useful to prepare GO with a high level of oxidation, hence a lower $\mathrm{C} / \mathrm{O}$ ratio (1.8)..$^{94,95}$

Co-oxidant: Alternatively, $\mathrm{K}_{2} \mathrm{FeO}_{4}$ of higher oxidability can enhance the intercalation and pre-oxidation of graphite and was used as a co-oxidant in combination with $\mathrm{KMnO}_{4}$. In this modified Hummers' method, a reduced amount of concentrated $\mathrm{H}_{2} \mathrm{SO}_{4}$ was used to increase the concentration of graphite and oxidants, which also improved the kinetics of the oxidation process. $^{96}$

Physical promoters: Hummers' method has also been modified in some cases to produce GO through the use of physical means to assist the in situ oxidative chemical exfoliation of graphite. Effectively, when ultrasonication (sonochemical), ${ }^{97,98}$ microwave irradiation $^{99}$ or ball milling techniques (mechanochemical) ${ }^{100}$ were applied directly to the reaction mixture, the delamination of Gr was favored and the yield of GO was increased compared to conventional stirring using a magnetic Teflon bar or rotary impeller to assist the exfoliation.

Safety: Tour and collaborators ${ }^{101}$ also notify that it is important to respect the mixing protocol in which, the graphite is added first to a mixture of sulfuric acid-phosphoric acid and finally, a

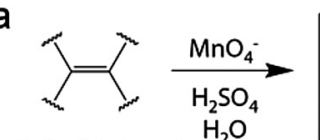

A double bond within GO

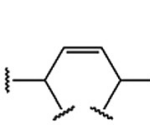

A double bond at the edge of $G O$
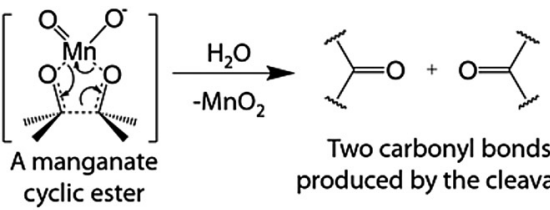

Two carbonyl bonds produced by the cleavage
C

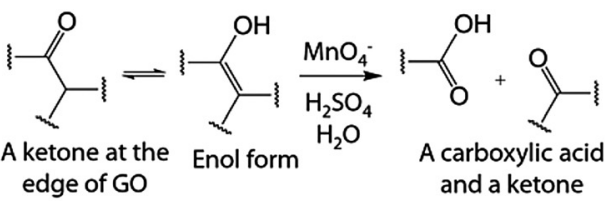

Scheme 6 Possible mechanisms of hidden step four in oxidation by Hummers' method: (a) oxidative cleavage of a C-C double bond via a manganese cyclic ester intermediately resulting in two carbonyl bonds, (b) oxidative cleavage of a C-C double bond producing two carboxylic acids, (c) oxidative cleavage of a ketone forming one carboxylic acid and one ketone, and (d) acid-catalyzed hydrolysis of an epoxy producing two hydroxyl bonds. Reprinted with permission from (Chem. Mater., 2016, 28, 756-764). Copyright (2016) American Chemical Society. DOI: 10.1021/acs.chemmater. 5b03700. Further permissions related to the material excerpted should be directed to the ACS. 


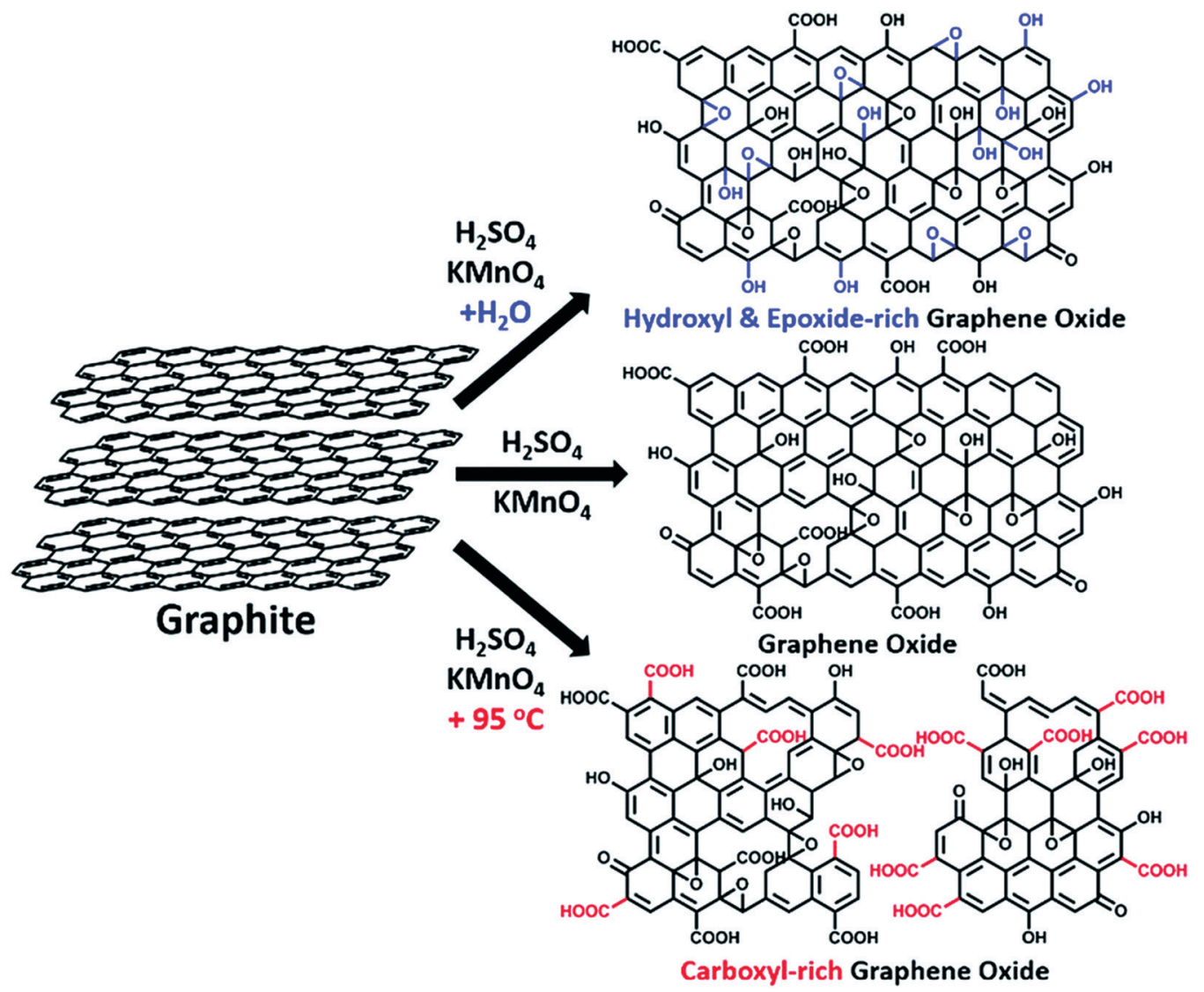

Fig. 17 The synthesis of GOs with controlled species of oxygenated groups. Reproduced from ref. 72 with permission from the Royal Society of Chemistry.

potassium permanganate is added in small portions to avoid any explosions or hazards caused by an excess of $\mathrm{KMnO}_{4}$ in the acid mixture. Also, a pre-cooling protocol (PCP) for mixing graphite at lower temperature $\left(5{ }^{\circ} \mathrm{C}\right)$ in the acid mixture to avoid explosions associated with the exotherm of the reaction on a larger scale has been developed. ${ }^{102}$ Mechanochemical techniques using much less acid and dry ice addition during the course of the reaction to keep the temperature under control to stabilize the highly oxidative and explosive $\mathrm{Mn}_{2} \mathrm{O}_{7}$ intermediate are effective methods to avoid safety risk. ${ }^{103}$

Low- and room-temperature methods: For low-cost production of GO on a large scale, it is important to put low- and roomtemperature oxidation into consideration due to the cost and risk for thermal input and less defective products obtained at lower temperatures. ${ }^{104-106}$ Eigler and collaborators have shown increasing advantages by performing the oxidation at temperatures below $10{ }^{\circ} \mathrm{C}$ for a longer time to minimize $\mathrm{CO}_{2}$ formation to yield highly intact GO with preserved structural integrity. ${ }^{104}$ However, this low-temperature process is time-consuming and suffers from low yield. ${ }^{105}$ A combination of a room-temperature oxidation procedure and a functionalization effect of water existing in commercial concentrated $\mathrm{H}_{2} \mathrm{SO}_{4}$ leads to $\mathrm{GO}$ with mainly hydroxyl and epoxy groups on the basal plane, helpful for exfoliation yield and dispersibility. This gives graphene material with fewer defects after reduction. ${ }^{105,106}$
Size and defects: In general, controlling the size and defects of GO sheets can be achieved by modifying the reaction parameters of the classical method: ${ }^{87,107-114}$ the type of graphite (large natural flakes, ${ }^{99,111}$ sieved flakes of various sizes, ${ }^{91,107,108}$ microparticles, ${ }^{23}$ powders ${ }^{111}$ ), the temperature (from 5 to $65^{\circ} \mathrm{C}$ ), ${ }^{104,112}$ the time (from a few minutes to a few days) and the stoichiometric amount of oxidant..$^{30,109,110,113,114}$ These parameters have an impact on the kinetics of the oxidation reaction, therefore they can be used to control the extent of oxidation which is in direct correlation with the size and defects of GO flakes. More precisely, because oxidative chemical exfoliation methods use harsh conditions acting like a "molecular shreader", they have a tendency to breakdown the carbon backbone and to yield GO flakes with smaller particle size than the initial graphite. ${ }^{109}$ Hence, heating the reaction at a more elevated temperature, for a longer time in the presence of more reagents drives the oxidation and likely yields small GO flakes with cracks and a high oxygen content (Fig. 18). ${ }^{109}$ To obtain more intact sheets, it is advantageous to use large natural flakes of graphite with higher crystallinity as a starting material because they are more resistant to the attack of strong acids and yield larger GO sheets with fewer defects. ${ }^{90,111}$ When discussing the factors affecting the size of GO flakes, mechanical stirring is also a parameter that cannot be ignored because it promotes the homogeneous mixture of graphite with oxidizing agents but 


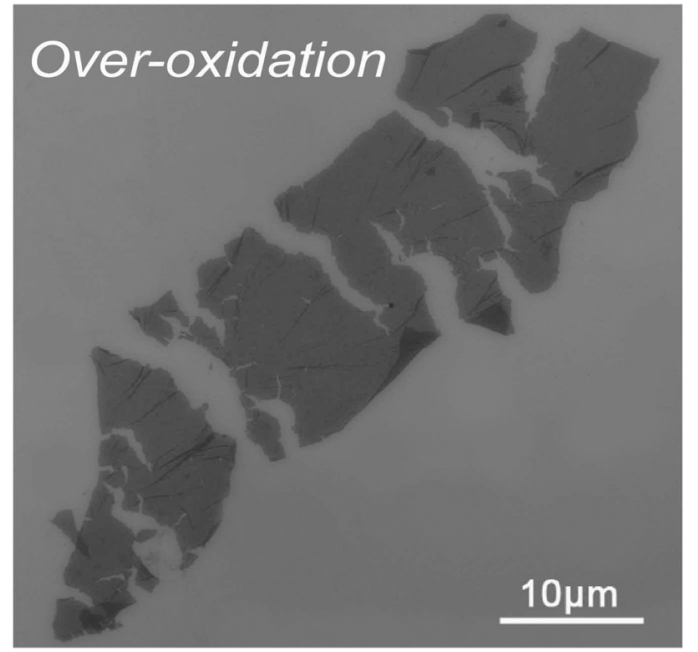

Fig. 18 SEM image of several pieces of GO sheets with a high oxygen content, showing that the small sheets come from the cracking of a big sheet. Reprinted (adapted) with permission from (ACS Nano, 2010, 4, 5245-5252). Copyright (2010) American Chemical Society.

introduces fragmentation in the GO flakes. For example, ultralarge GO sheets can be obtained from an agitation-free modified Hummers' oxidation. ${ }^{115-117}$

For GO papers and membranes, it is clear that large GO flakes induce stronger mechanical performance, higher electrical/thermal conductivity and stronger electromagnetic interference shielding performance. For example, by comparing the properties of GO papers made from small (S) and large (L) flakes, LGO papers exhibit $320 \%$ improvement in Young's modulus and $280 \%$ improvement in tensile strength. In addition, when made into a monolithic porous material, the LGO-based material performs better than SGO in terms of electrical conductivity, mechanical strength and ionabsorption. For electrochemical applications, increasing the lateral size is beneficial to the electrical conductivity and stability of graphene-based anodes, which contributes to the improved cycle stability of Li/Al-ion batteries. Further evidence comes from the fact that LGO-based networks perform better in $\mathrm{Li} / \mathrm{S}$ batteries than their SGO counterparts. ${ }^{116}$

4.1.1.3 Novel oxidants. Beside chlorate and permanganatebased oxidizers, modern ways to oxidize graphite include the use of potassium chromate under the Jones conditions ${ }^{7,118}$ or in combination with nitric or perchloric acid. ${ }^{119}$ Also, GO can be prepared using less toxic potassium ferrate in sulfuric acid at room temperature, ${ }^{120}$ although it is not generally recognized that ferrate ions $(\mathrm{Fe}(\mathrm{vI})$ ) are suitable for the oxidation of graphite because of their high instability in an acidic environment and low oxidation power in neutral and alkaline environments. ${ }^{121}$ Alternatively, $\mathrm{Fe}(\mathrm{vI})$ can oxidize graphite in water in the presence of $\mathrm{H}_{2} \mathrm{O}_{2}$ with heating at $50{ }^{\circ} \mathrm{C} .{ }^{122}$ Interestingly, GO quantum dots (GO-QDs) can be prepared from the acid-free oxone oxidation of carbon structures such as graphite, carbon nanotubes, carbon fibers and charcoal in DMF. ${ }^{123}$ Similarly, benzoyl peroxide (BPO) can oxidize graphite at $110{ }^{\circ} \mathrm{C} .{ }^{124}$ Noteworthily, BPO acts as the sole oxidant and reagent towards graphene oxide under these metal-free conditions. In general, the new state-of-the-art developments in the wet preparation of GO are purposed to replace the more toxic and hazardous methods such as the ones previously presented based on chlorate or permanganate oxidation. Those processes using novel oxidants are considered scalable, but the cooling and handling of large volumes of hot solutions still bring several manufacturing problems not easy to solve. In terms of quality, the GO produced through chemical methods suffers from the presence of impurities, and more importantly, from significant structural damage as a result of the harsh synthetic conditions. Both of these features cause alteration of its electrical properties, which limits its use for electronic devices. However, the presence of such defects can be beneficial for tailoring GO electrochemical behavior.

4.1.2 Electrochemical methods. Top-down methods are of key importance for large-scale graphene oxide preparation, especially the ones using electrochemistry (E) to promote the oxidation and exfoliation of graphite into EGO. They are considered to be more environmentally friendly than the chemical methods presented in section 4.1.1 particularly on the demonstration of re-using the electrolyte multiple times and minimum washing, hence generating little waste. ${ }^{125,126}$ More importantly, the scalability of the electrochemical method has been demonstrated by directly employing natural flake graphite (instead of a processed graphite foil electrode) in a packed-bed reactor ${ }^{127}$ or a stirred vessel. ${ }^{125}$ The most important factor to explain the better quality of EGO compared to GO-BR, GO-ST, GO-HO, GO-HU and GO-TO comes from the use of aqueous electrolytes avoiding the introduction of residual impurities in the materials. ${ }^{126}$ Furthermore, the modifications of the experimental set-up ${ }^{128}$ (Fig. 19) (electrolytes, voltage, temperature, time, geometry of the electrode, etc.) during the process generates EGO with different densities of defects and also with different levels of oxidation (see Table 1). In addition, the oxidized functional groups $(\mathrm{C}-\mathrm{O})$ or $(\mathrm{C}=\mathrm{O})$ on
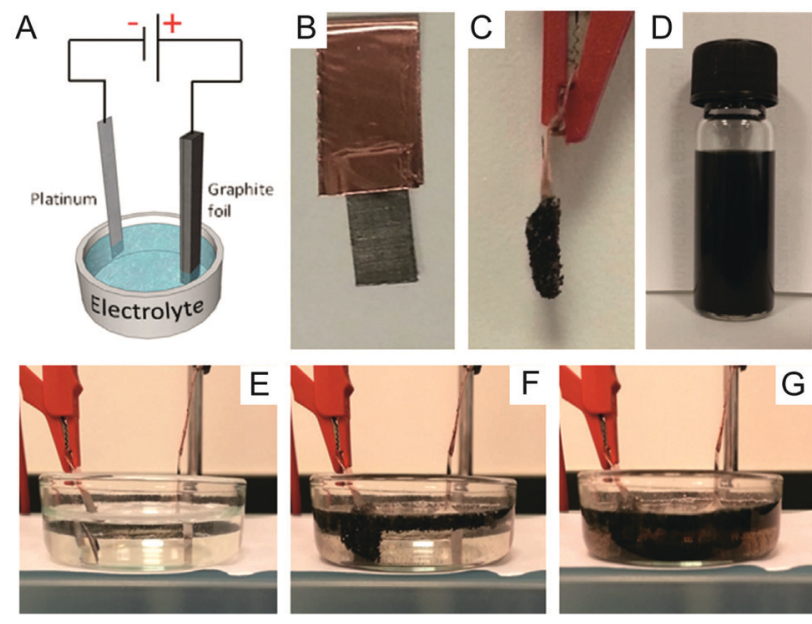

Fig. 19 (A) Schematic illustration of the experimental setup. Photos of graphite foil (B) before and (C) after the exfoliation process. (D) Graphene dispersion in DMF solution (1 $\mathrm{mg} \mathrm{mL}^{-1}$ ). Photos illustrating the exfoliation process at (E) time zero, (F) after $5 \mathrm{~min}$, and (G) after $20 \mathrm{~min}$. Reproduced from ref. 128 with permission from Wiley-VCH. 
Table 1 List of methods for the oxidative exfoliation of graphite towards GO

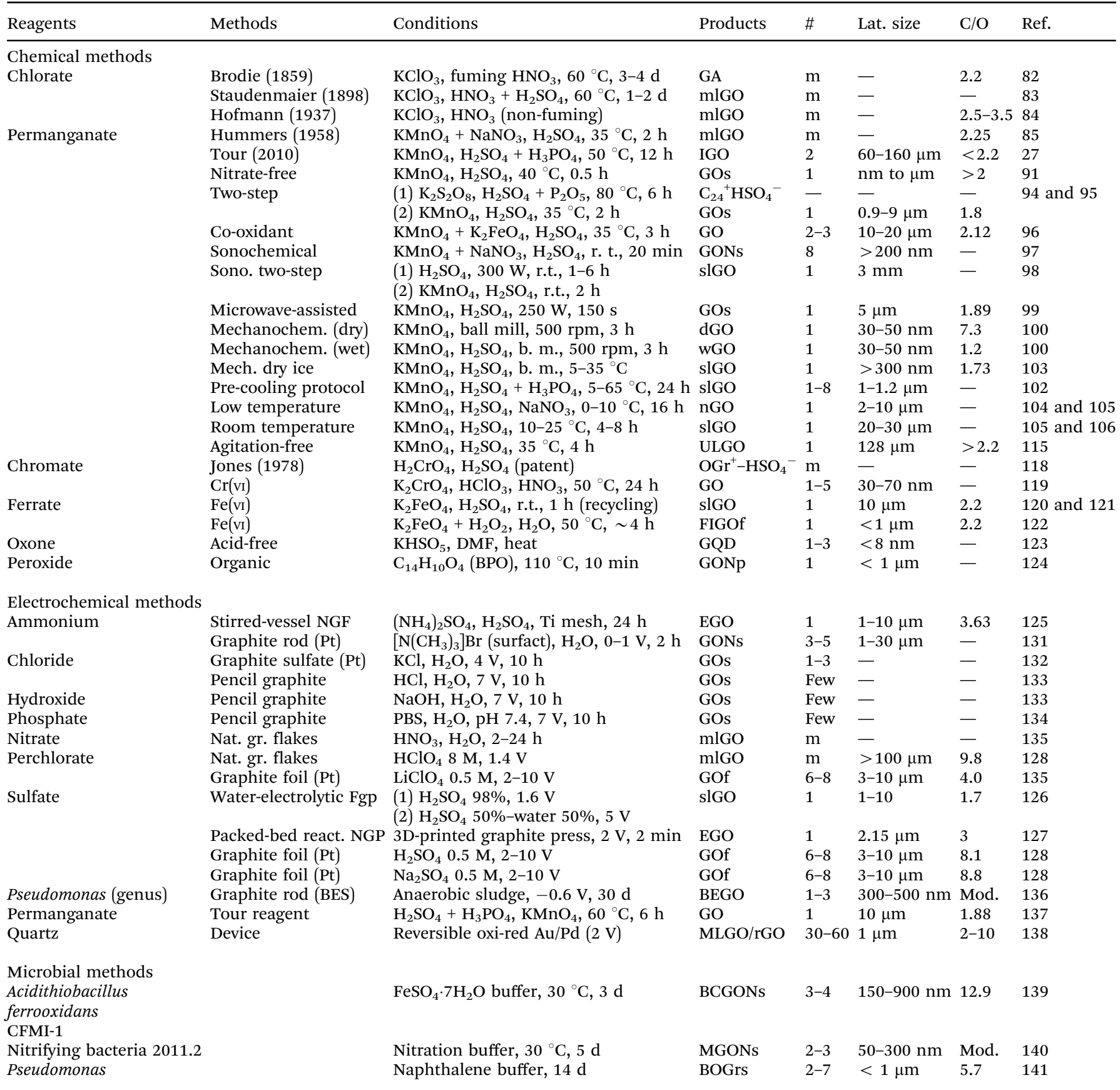

$\#$ = number of layers; $\mathrm{GA}=$ graphic acid; $\mathrm{m}=$ many; $\bmod .=$ moderate; $\mathrm{ml}=$ multi lamellar; $\mathrm{GO}=$ graphene oxide; $\mathrm{s}=$ sheet; $\mathrm{I}=$ improved; $\mathrm{Np}$ = nanoplatelet; $\mathrm{Ns}=$ nanosheet; $\mathrm{OGr}$ = oxidized graphite; sl = single layer; FI = ferro-induced; $\mathrm{QD}=$ quantum dot; $\mathrm{f}=$ flake; $\mathrm{d}=\mathrm{dry} ; \mathrm{w}=\mathrm{wet} ;$ $\mathrm{n}=$ new; UL = ultra large; NGF = natural graphite flake; Fgp = flexible graphite paper; $\mathrm{BES}=$ bioelectro system; $\mathrm{BE}=$ bioelectrochemically exfoliated; $\mathrm{BC}=$ biologically converted; $\mathrm{M}=$ microbial; $\mathrm{B}=$ bacteria-treated.

EGO can be tuned simply via the electrolyte concentration. ${ }^{129}$ Quinones, hydroxyls, aldehydes, epoxides, and peroxide functional groups are inherently electrochemically active, showing both oxidizability and reducibility at mild electrochemical potentials. At more extreme potentials and $\mathrm{pH}$, it is also possible to reduce carboxyl groups (lower than $-2 \mathrm{~V}$ ). It is interesting to note that different preparation methods of GO, such as those of Staudenmaier, Hofmann, Hummers, or Tour, demonstrate contrasting reduction potentials. Specifically, GO-ST and GO-HO exhibit a single reduction wave at $-1.2 \mathrm{~V}$ (vs. $\mathrm{Ag} / \mathrm{AgCl})$ and GO-HU shows three distinctive waves $(-1.2,-1.4$, and $-1.8 \mathrm{~V}$ vs. $\mathrm{Ag} / \mathrm{AgCl}$ ). On the other hand, GO-TO exhibits superposed waves at -1.4 and $-1.8 \mathrm{~V} .^{130}$

Electrochemical methods have gained attention recently because they are performed in water and they are based on a fast and simple process easy to execute. Electrochemistry consists 
of driving electrons into the graphite electrode (foil, rod or flakes, flexible paper) under anodic or cathodic conditions to force the intercalation of aqueous electrolyte ions in the interlayer galleries of graphite, hence, driving its exfoliation as a consequence. ${ }^{125,128}$ Several different electrolytes such as ammonium, ${ }^{131}$ chloride, ${ }^{132,133}$ hydroxide, ${ }^{133}$ phosphate ${ }^{134}$ nitrate, ${ }^{135}$ perchlorate, ${ }^{128,135}$ sulfate ${ }^{128}$ and genus Pseudomonas in anaerobic sludge ${ }^{136}$ have been tested for the preparation of mono and few-layer EGO. Water (W) electrolytic oxidation of graphite sulfate ${ }^{126}$ is an extremely clean and fast process. After absolute control of the experimental set-up, using a lifting platform to move slowly the graphite flexible paper (GFP) pre-intercalated electrochemically with sulfuric acid, W-EGO having a C/O ratio (1.7) lower than GO-TO (1.8) is obtained in high yield with few defects. It is worth noting that the intensity ratio of the D peak to G peak in W-EGO is higher than that in GO-HU, suggesting that the $\mathrm{sp}^{2}$ domains in W-EGO are smaller than those in GO-HU. With only two $250 \mathrm{~mL}$ beakers as reactors and a $0.5 \mathrm{~mm}$-thick, $40 \mathrm{~mm}$-wide FGP as the raw material, this method yields W-EGO at a rate of about $12 \mathrm{~g} \mathrm{~h}^{-1}$. Interestingly, the water electrolytic method involved minimum washing and re-using of the electrolytes, showing the great potential of this method for mass production of EGO sheets (Fig. 20). ${ }^{126}$

Another two-step approach for the synthesis of large graphene oxide (LGO) sheets with lateral dimensions of $\approx 10 \mu \mathrm{m}$ or greater has been developed. ${ }^{137}$ The first step is a pre-treatment step involving electrochemical intercalation of $\mathrm{H}_{2} \mathrm{SO}_{4}$ and $\mathrm{H}_{3} \mathrm{PO}_{4}$, the mixture of acids used in the Tour method. This causes exfoliation of the graphene layer and increases the interlayer spacing between the sheets. The second step is the conventional oxidation step, where oxidation of exfoliated graphene sheets was performed using $\mathrm{KMnO}_{4}$ as the oxidizing agent. Electrochemistry is a powerful tool as it allows GO to be reversibly reduced and oxidized using electrical stimulus. Controlled reduction and oxidation in two-terminal devices containing multilayer GO films have shown to result in switching between partially reduced EGO and ErGO, a process which modifies the electronic and optical properties (Fig. 21). ${ }^{138}$

4.1.3 Microbial methods. Nowadays, graphitic materials oxidized by biological systems are of great importance for practical applications because microbial methods involve usually ecofriendly and low-cost techniques. After being cultivated with bacteria, graphite is not homogeneously oxidized, and moreover, GO sheets are exfoliated from the bulk and suspended in the medium. To date, works done on the bio-oxidation of graphite have shown that the contact between bacterial cells and the graphitic materials promotes the electron transfer, and hence the oxidation. For example, Acidithiobacillus ferrooxidans can oxidize graphite after a 3-day exposure at $30{ }^{\circ} \mathrm{C}$ in a $\mathrm{FeSO}_{4} \cdot 7 \mathrm{H}_{2} \mathrm{O}$ buffer. ${ }^{139}$ Also, graphite can afford GO after 5 days in a nitration buffer in the presence of nitrifying bacteria $\left(30{ }^{\circ} \mathrm{C}\right) .{ }^{140}$ Biotransformations are slow processes and yield however multilayered GO materials with a low level of oxidation (C/O: 5-12). In the case of Pseudomonas, a naphthalene degrading bacterium,

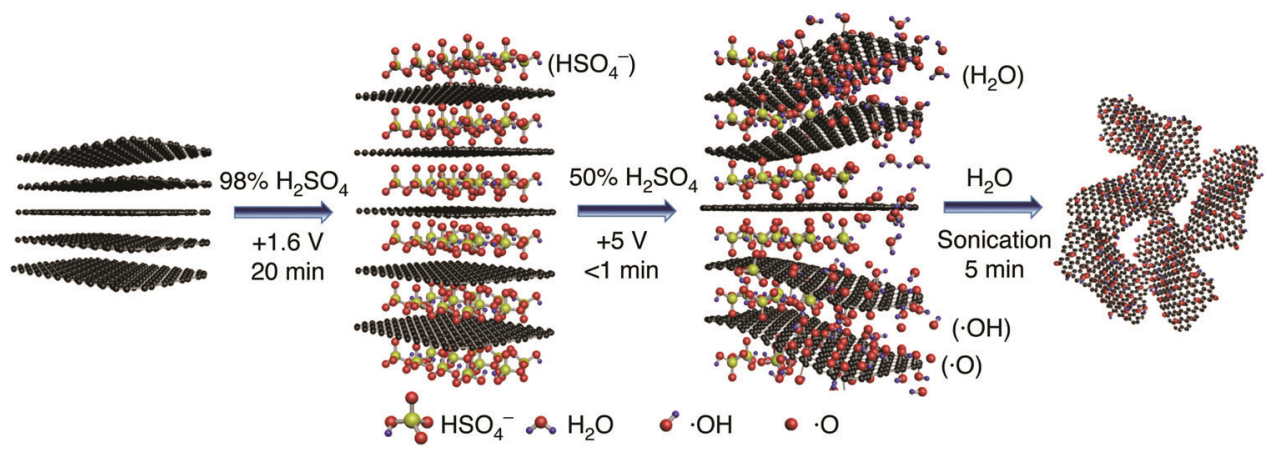

Fig. 20 Synthesis of EGO by water electrolytic oxidation: schematic illustration of the synthesis process of EGO by water electrolytic oxidation. Reproduced from ref. 126 with permission from the NPG. http://creativecommons.org/licenses/by/4.0/.

A

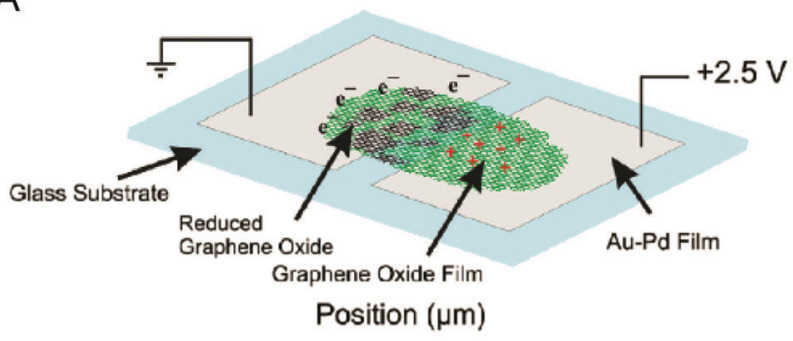

B

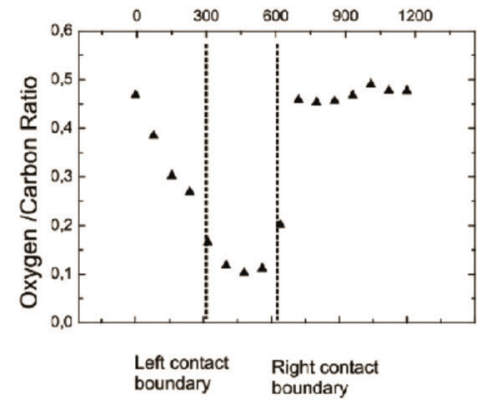

Fig. 21 (A) Thin multilayer graphene oxide films (30-60 nm thickness) can be electrically reduced and oxidized. The two-terminal device is schematically shown. (B) Oxidation and reduction are confirmed by XPS measurements. The oxygen-to-carbon ratio is measured using a $100 \mu \mathrm{m}$ diameter photoelectron collection spot and is plotted as a function of distance across the device. The data show gradual changes in the stoichiometry between the contacts. Reprinted with permission from (ACS Nano, 2011, 5, 2475-2482). Copyright (2011) American Chemical Society. 

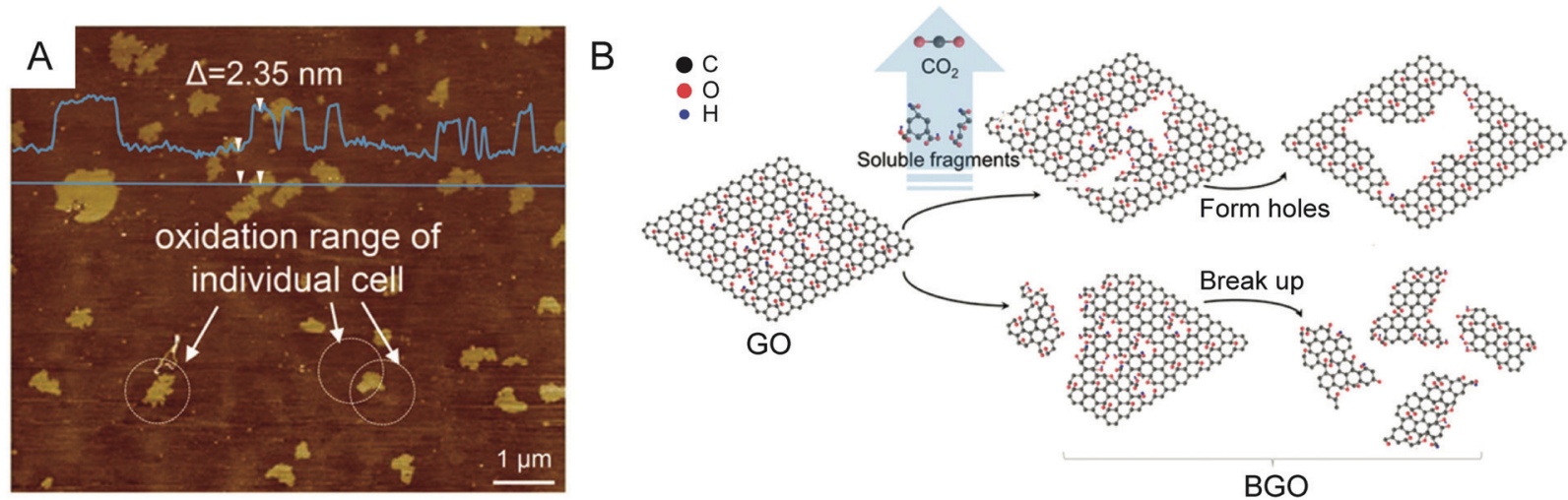

Fig. 22 (A) Photos of graphite foil after the exfoliation process. (B) Schematic diagram for bacterial degradation of GO. Reproduced from ref. 141 with permission from the Royal Society of Chemistry.

the transformation is found to be effective at producing oxidized graphitic materials after 14 days of incubation. The range of effective oxidation is likely to be limited by the size of bacterial cells. A single bacterial cell comes into contact with graphite within a circle whose diameter is about $1 \mu \mathrm{m}$ (Fig. 22). The same surface area may be exposed to oxidation many times and the most heavily oxidized areas will be at the intersections of these circles, whose diameters are less than $1 \mu \mathrm{m}$. Therefore, graphite with an average particle size of $122 \mu \mathrm{m}$ can't be oxidized uniformly and some heavily oxidized areas exfoliate from the sheets, forming GO. For the bacterial degradation of GO, the same reason probably leads to the formation of holes, the size of which is also below $1 \mu \mathrm{m}$ (Fig. 22). ${ }^{141}$

\subsection{Oxidative chemical methods for 3D carbon structures}

The chemical methods used to oxidize and exfoliate pristine graphite or natural graphite flakes presented in Section 4.1 are approaches that are flexible and therefore can be generalized to a wider scope of carbon sources. Using the peroxide, persulfate or permanganate method, alone or in combination with a physical means to promote the delamination, GO can be prepared successfully from other carbon precursors with three dimensional structures such as carbon black, ${ }^{142}$ carbon fibers, ${ }^{143}$ carbon nanohorns, ${ }^{144,145}$ carbon nanotubes, ${ }^{146}$ ${ }^{13} \mathrm{C}$-labeled CVD-graphite grown on nickel ${ }^{13}$ and expanded graphite (Fig. 23). ${ }^{147-149}$ The advantages given by these methods are to allow a higher reactivity of the carbon source and to control the formation of GO with new morphological shape or with different isotopic compositions. For example, the hydrothermal oxidation of carbon black by hydrogen peroxide yields spherical quantum nanodots of GO with diameters of around 3 to $4.5 \mathrm{~nm} .{ }^{142}$ Also, persulfate and permanganate oxidation of carbon fibers yields single layer GO sheets of up to $1 \mu \mathrm{m}$ in length, ${ }^{143}$ while nanosheets of $\mathrm{GO}(2-50 \mathrm{~nm})$ can be prepared from
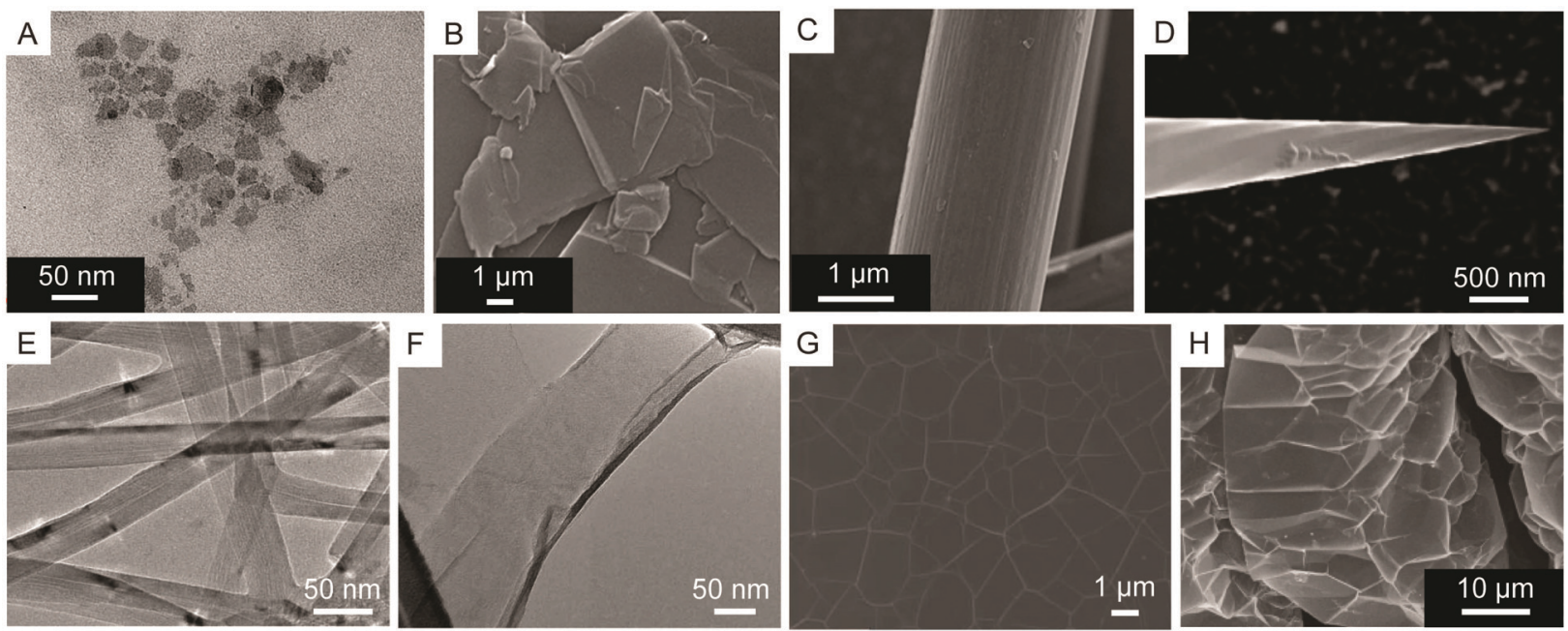

Fig. 23 Electron microscopy images of (A) GO produced from amorphous carbon black. Reproduced (adapted) from ref. 142 with permission from the Royal Society of Chemistry. (B) Natural graphite flakes and (C) carbon fibers. Reproduced (adapted) from ref. 143 with permission from the NPG (http:// creativecommons.org/licenses/by/4.0/). (D) Carbon nanohorns. Reprinted with permission from (Chem. Rev., 2016, 116, 4850-4883). Copyright (2016) American Chemical Society. (E) Carbon nanotubes and (F) GO nanoribbons from the unzipping of a carbon nanotube. Reproduced (adapted) from ref. 146 with permission from the NPG (http://creativecommons.org/licenses/by/4.0/). (G) ${ }^{13} \mathrm{C}$-graphite grown on nickel (CVD). From [Science, 2008, 321, 1815-1818]. Reprinted (adapted) with permission from AAAS. (H) Expanded graphite. Reproduced (adapted) from ref. 149 with permission from the Elsevier Science. 
Table 2 List of carbon precursors towards GO

\begin{tabular}{|c|c|c|c|c|c|c|c|}
\hline Carbon precursors & Methods & Conditions & Products & \# & Lat. size & $\mathrm{C} / \mathrm{O}$ & Ref. \\
\hline Carbon black & Peroxide & $\mathrm{H}_{2} \mathrm{O}_{2} 1 \%$, autoclave, $180{ }^{\circ} \mathrm{C}, 1.5 \mathrm{~h}$ & GO-dot & $3-8$ & $3-4.5 \mathrm{~nm}$ & 1.84 & 142 \\
\hline Carbon nanohorns & Mod. Hummers & $\mathrm{KMnO}_{4}, \mathrm{H}_{2} \mathrm{SO}_{4}, 70{ }^{\circ} \mathrm{C}, 40 \mathrm{~min}$ & GONs & $1-10$ & $2-50 \mathrm{~nm}$ & - & 144 \\
\hline Carbon nanotubes & Unzipping (MW) & $\begin{array}{l}\text { (1) } \mathrm{H}_{2} \mathrm{SO}_{4} \text {, r.t., } 24 \mathrm{~h} \\
\text { (2) } \mathrm{KMnO}_{4} \text {, r.t., } 1 \mathrm{~h} ; 55-70{ }^{\circ} \mathrm{C}, 1 \mathrm{~h}\end{array}$ & GONr & $1-3$ & $0.1-4 \mu \mathrm{m}$ & 1.38 & 146 \\
\hline & Microwave (3 steps) & $\begin{array}{l}\text { (1) sealed tube, } \mathrm{N}_{2},<2 \mathrm{~s} \\
\text { (2) } \mathrm{K}_{2} \mathrm{~S}_{2} \mathrm{O}_{8}, \mathrm{H}_{2} \mathrm{SO}_{4}+\mathrm{P}_{2} \mathrm{O}_{5}, 80{ }^{\circ} \mathrm{C}, 4.5 \mathrm{~h} \\
\text { (3) } \mathrm{KMnO}_{4}, \mathrm{H}_{2} \mathrm{SO}_{4}, 35{ }^{\circ} \mathrm{C}, 2 \mathrm{~h}\end{array}$ & slGO & 1 & $11-14 \mu \mathrm{m}$ & - & 148 \\
\hline \multicolumn{8}{|c|}{ Oxidation methods for graphene } \\
\hline \multirow[t]{2}{*}{ CVD graphene } & Photochemical & UV light, $\mathrm{O}_{3}$, r.t., atm $\mathrm{P}$ & slOG & 1 & - & High & 150 \\
\hline & Rf Plasma & $\mathrm{O}_{2}, 15 \mathrm{~W}, 20$ mTorr & slOG & 1 & - & High & 151 \\
\hline Coronene & Catalytic growth & $316 \mathrm{SS}, \mathrm{H}_{2}, \mathrm{~N}_{2}, \mathrm{TCB}, 800{ }^{\circ} \mathrm{C}$ & rGO film & $9-23$ & - & Mod. & 156 \\
\hline Ethanol & CVD-repair & $\mathrm{GO}, \mathrm{H}_{2}, 950{ }^{\circ} \mathrm{C}$ & rGO film & $<5$ & - & - & 157 \\
\hline Ethylene & CVD-repair & $\mathrm{rGO}, 800{ }^{\circ} \mathrm{C}, 3 \mathrm{~min}$ & CVDGO & 1 & $>0.1-10 \mu \mathrm{m}$ & - & 158 \\
\hline Isopropanol & CVD-repair & $\mathrm{rGO}, \mathrm{H}_{2}, \mathrm{Ar}, 750{ }^{\circ} \mathrm{C}$ & rGO & 1 & $\sim 30 \mu \mathrm{m}$ & - & 159 \\
\hline \multicolumn{8}{|c|}{ Hydrothermal methods for carbohydrates } \\
\hline Glucose (fructose, sucrose) & Tang-Lau & $\mathrm{H}_{2} \mathrm{O}$, autoclave, $160{ }^{\circ} \mathrm{C}, 30 \mathrm{~min}$ & GOs & $1-1500$ & $20-100 \mu \mathrm{m}$ & 2.8 & 160 \\
\hline Glucose & Tang-Lau & $\mathrm{H}_{2} \mathrm{O}$, microwave, $595 \mathrm{~W}, 1-9 \mathrm{~min}$ & GQDs & $<5 \mathrm{~nm}$ & (spheres) & 20.7 & 161 \\
\hline \multicolumn{8}{|c|}{ Thermal decomposition methods for organic matter rich in $\mathrm{C}$} \\
\hline Bamboo & Two-steps pyrolysis & $673-973 \mathrm{~K}, \mathrm{~N}_{2}$ & OGNp & $25-60$ & $5-100 \mu \mathrm{m}$ & $7-32$ & 171 \\
\hline Rice straw biochar & $\begin{array}{l}\text { (1) Pyrolysis } \\
\text { (2) Mod. Hummers }\end{array}$ & $\begin{array}{l}450{ }^{\circ} \mathrm{C}, \mathrm{N}_{2}, 45 \mathrm{~min} \\
\mathrm{KMnO}_{4}, \mathrm{H}_{2} \mathrm{SO}_{4} \text {, r.t., } 20 \mathrm{~min}\end{array}$ & GONp & $1-3$ & $60-100 \mathrm{~nm}$ & - & 172 \\
\hline Sugarcane & Catalytic pyrolysis & Ferrocene, $300{ }^{\circ} \mathrm{C}, 10 \mathrm{~min}$ & mlGO & $\mathrm{m}$ & - & - & 173 \\
\hline
\end{tabular}

$\#$ = number of layers; $\mathrm{GO}=$ graphene oxide dot = quantum dot; $\mathrm{sl}=$ single layer; $\mathrm{Ns}=$ nanosheet; $\mathrm{Nr}=$ nanoribbon; $\mathrm{ml}=$ multi lamellar; OG = oxidized graphene; r.t. $(\mathrm{RT})=$ room temperature; $\mathrm{EG}=$ expanded graphite; $\mathrm{B}=$ bacteria-treated; $\mathrm{rGO}=$ reduced graphene oxide; $\mathrm{CVD}=$ chemical vapor deposition; $\mathrm{s}$ = sheet; $\mathrm{Np}=$ nanoplatelet; $\mathrm{m}$ = many; mod. = moderate; $\mathrm{GQG}=$ graphene quantum dot.

carbon nanohorns using modified Hummers' method ${ }^{144}$ and the unzipping of multiwalled (MW) carbon nanotubes by successive treatments with $\mathrm{H}_{2} \mathrm{SO}_{4}$ and $\mathrm{KMnO}_{4}$ yields $\mathrm{GO}$ nanoribbons with controllable size ranging from $100 \mathrm{~nm}$ to $4 \mu \mathrm{m} .{ }^{146}$ One important example shows that GO enriched with carbon-13 can be prepared from modified Hummers' method using ${ }^{13} \mathrm{C}$-graphite prepared using CVD techniques as the carbon source. ${ }^{13}$ Finally, expanded graphite is commercially available and is another convenient source of carbon more reactive than conventional graphite towards the oxidation using Hummers' method. In the presence of the intercalating agents $\left(\mathrm{H}_{2} \mathrm{SO}_{4}\right)$, expanded graphite goes into a spontaneous expansion step and produces a foam-like intermediate more easily oxidized by the oxidant $\left(\mathrm{KMnO}_{4}\right)$. This method becomes facile, safe, productive and practical for scalable production of larger flakes of GO (Table 2). ${ }^{147-149}$

\subsection{Oxidative methods for graphene}

The development of practical methods appropriate for the production of graphene and GO with a high control of the atomic structure is mandatory for the fabrication of devices with sub-nanometer precision and is an important step toward the miniaturization as it should facilitate applications in the areas of nanoelectronics, molecular sensors, photonics, catalysis and composite materials. GO-based devices can be made directly from CVD graphene using nanopatterning techniques in combination with an oxidative method to change the level of oxidation at specific locations. Oxidized graphene with a low level of oxidation can be obtained by photochemical oxidation in the presence of UV and $\mathrm{O}_{2}{ }^{150}$ oxygen plasma ${ }^{151}$ and local anodic oxidation using a conductive AFM (atomic force microscope) tip. ${ }^{152}$ However, a fully comprehensive, systematic study of the impact of those methods on graphene is still missing in the literature. The extent of oxygen functionalization (in terms of oxygen density) is controlled by careful tuning of the time duration of the treatments. In the case of AFM, a negative bias voltage of 15 to $30 \mathrm{~V}$ is applied on the silicone tip, creating an electric field large enough to induce electrochemical oxidation of the sample at room temperature in the presence of moisture. Lithography is capable of producing small features with sub-nanometer spatial resolution $(25 \mathrm{~nm})$. The process allows controlling the dimensions and electrical conductivity during the fabrication (Fig. 24).

\subsection{CVD methods for hydrocarbons}

Recently developed chemical vapor deposition techniques have emerged as one of the most popular ways for the scalable 

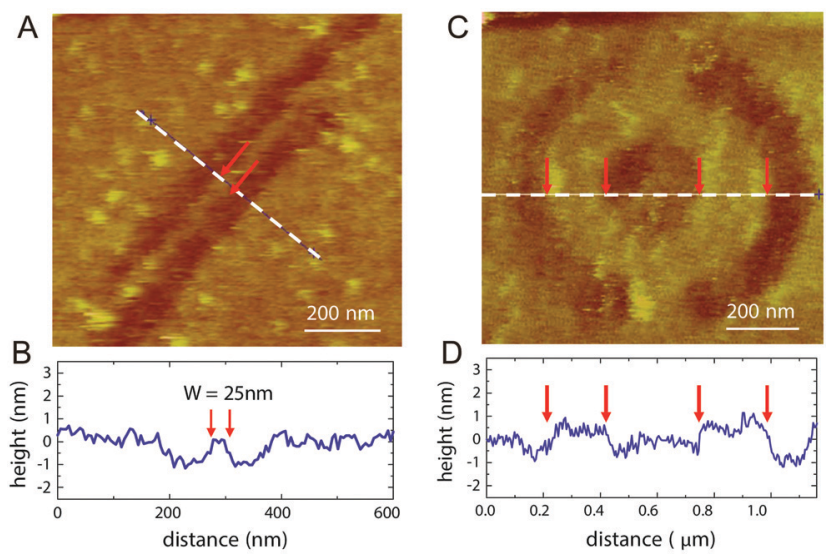

Fig. 24 (A) AFM image of a nanoribbon fabricated on a graphene flake with thickness $\sim 1 \mathrm{~nm}$. The width and length of the ribbon are 25 and $800 \mathrm{~nm}$, respectively. (B) Height profile along the dashed line in (a). (C) A nanoring (inner radius $\sim 160 \mathrm{~nm}$, outer radius $\sim 380 \mathrm{~nm}$ ) patterned on a graphene flake. Two long trenches, not shown in the picture, were subsequently drawn from the circumference of the ring outward to the edges of the flake to electrically isolate the ring device. (D) Height profile along the dashed line in (c). Reproduced from ref. 152 with permission from the AIP Publishing.

production of large-area and high-quality carbon materials for various applications in electronics, because they are inexpensive, versatile and they produce materials of very high purity. CVD is extremely useful in the process of atomic layer deposition of monocrystalline, polycrystalline, amorphous, and epitaxial materials such as graphene, carbon nanofibers and nanotubes, diamond, fluorocarbons and more recently, graphene oxide and reduced graphene oxide. ${ }^{153}$

The CVD technique. In a typical CVD experiment for the synthesis of graphene, ${ }^{154}$ a hydrocarbon source is atomized, either thermally or via plasma chemistry, over a transition metal-based catalytic surface. Following the decomposition of the precursor into active carbon species, the reorganization of the elements on the surface of the substrate yields more complex solid materials. During the deposition, the metal surface (substrate) not only works as a catalyst to lower the energy barrier of the reaction, but also determines the nature of the mechanism. In the case of metals having high carbon solubility such as $\mathrm{Ni}$ and $\mathrm{Fe}$, the carbon will dissolve and diffuse into the heated substrate according to its solubility. Upon cooling, the dissolved carbon will segregate to form graphene sheets. On the other hand, for metals with low carbon solubility such as $\mathrm{Cu}$, carbon atoms will nucleate and laterally expand around the nucleus to form graphene domains. The growth process is referred to as a "self-limited surface deposition" growth mechanism and will terminate when the substrate is fully covered with the graphene layer. Noteworthily, volatile by-products are also produced, which are removed easily by gas flow through the reaction chamber (Fig. 25). ${ }^{155} \mathrm{GO}$ and rGO (reduced graphene oxide) films can be prepared from methane using thermal $\mathrm{CVD}$ at $500{ }^{\circ} \mathrm{C}$ on copper foil $(\mathrm{Cu})^{155}$ or from coronene at $800{ }^{\circ} \mathrm{C}$ on stainless steel $(\mathrm{Ni}-\mathrm{Fe}) .{ }^{156} \mathrm{CVD}$ experiments were achieved in the presence of hydrogen $\left(\mathrm{H}_{2}\right)$ and carrier gases such as $\mathrm{N}_{2}$ or Ar. The presence of the oxo

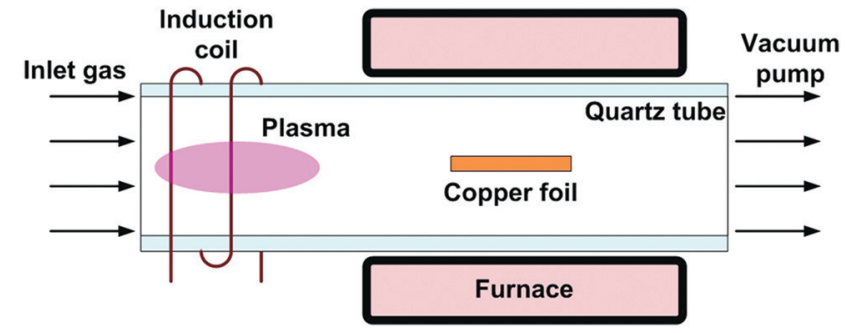

Fig. 25 Layout of the PECVD system. Reproduced from ref. 154 with permission from the AIP Publishing.

functionalities in GO (or rGO) obtained from CVD were introduced through the residual gases containing oxygen or moisture traces. In the CVD work using coronene on stainless steel substrates, ${ }^{156}$ multilayer rGO films with a moderate level of oxidation were obtained. On the other hand, plasma-enhanced (PE) CVD of methane on copper foil gave $\mathrm{GO}$ with a lower $\mathrm{C} / \mathrm{O}$ ratio $(1.6-3.2),{ }^{155}$ very similar to the values obtained with chemical methods.

Defect repairing. Besides metal substrates, GO synthesis on insulating substrates has been studied using graphene oxide ${ }^{157}$ itself and its reduced form, rGO, ${ }^{158,159}$ to catalyze the thermal CVD of ethanol, ${ }^{157}$ ethylene ${ }^{158}$ and isopropanol. ${ }^{159}$ The extended CVD growth mechanism is referred to as "CVD repairing", and usually involves the surface adsorption, nucleation and coalescence of the atomized elements into the holes and defects found in the GO or rGO substrates (Fig. 26). Without the catalytic effect of a transition metal substrate, mono and few-layer GO are deposited on the surface. Higher decomposition temperatures are usually required $\left(750-900{ }^{\circ} \mathrm{C}\right)$ compared to catalytic methods using transition metals. Therefore, the materials prepared with insulating substrates undergo in situ thermal decomposition ${ }^{23}$ yielding mostly rGO films with a lower level of oxidation. After the restoration of the $\mathrm{sp}^{2}$-carbon lattice, the materials have lower defect density with improved conductivity. The authors claimed that the reparation contributed to increase the charge mobility in GO and also to enhance carrier delocalization due to the decrease in the defect areas. ${ }^{159}$ CVD-based graphene layers are of large size, high-quality and they are important for optoelectronic or microelectronic devices. However, for applications in the field of electrochemical power sources and composites, this technique is limited due to the low amount of materials produced. ${ }^{37}$

\subsection{Hydrothermal methods for carbohydrates}

Large GO flakes. In 2012, Tang and collaborators ${ }^{160}$ developed a novel bottom-up synthesis approach to fabricate GO using a hydrothermal method employing carbohydrates such as glucose, fructose or sucrose as a sole reagent. The method is environmentally friendly, facile, low-cost as well as capable of scaling up for mass production. It is a self-assembly method to synthesize GO nanosheets with tunable thickness ranging from $1 \mathrm{~nm}$ (monolayer) to $1500 \mathrm{~nm}$. The lateral sizes of the monolayer and few-layer $(<5)$ GONs are about $20 \mu \mathrm{m}$ and $100 \mu \mathrm{m}$ respectively. It is explained using a layer-by-layer bottom-up mechanism. Under hydrothermal 
Before Growth

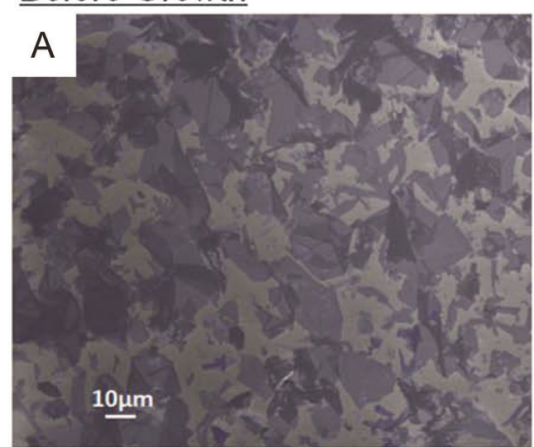

After Growth

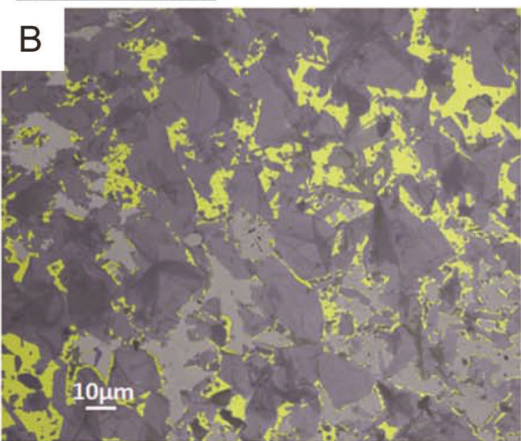

\section{High Magnification}

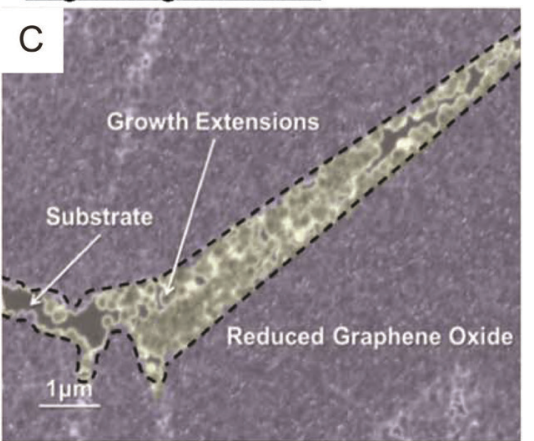

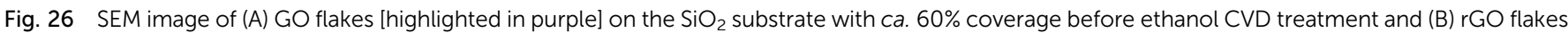

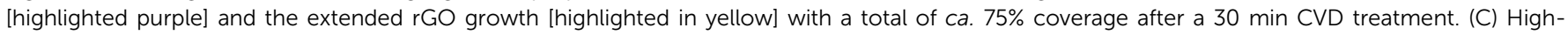

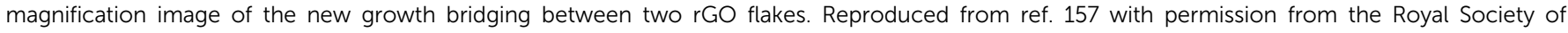
Chemistry.

conditions, carbohydrate molecules undergo cyclic polymerization to form monolayer GO, which floats on solution due to its hydrophobicity. The second GO layer may be grown underneath the first GO layer forming a bi-layer. Subsequently, tri-layer and multilayer GO nanosheets are produced (Fig. 27).

Q-Dots and doped-Q-Dots. Investigating the potential of carbohydrate chemistry for the development of graphene-based materials with other properties, using hydrothermal carbonization (HTC) in combination with microwave irradiation, Tang and collaborators have also reported the preparation of graphene quantum dots (GQDs) smaller than $5 \mathrm{~nm}$ containing oxo functionalities with a high C/O ratio (20.7). ${ }^{161}$ HTC methods are flexible methods that allow incorporation of heteroatoms into the GQD structure such as $\mathrm{B}, \mathrm{Cl}, \mathrm{Mn}, \mathrm{N}$ and $\mathrm{S}$ by the addition of boric acid, ${ }^{162}$ hydrochloric acid, ${ }^{40}$ ammonia $^{163,164}$ for urea, ${ }^{165}$ manganese chloride $^{166}$ or sulfuric acid ${ }^{167}$ in the reaction mixture. Quantum dots are small particles $(\sim 10 \mathrm{~nm})$ and they arise by controlling the temperature, the concentration and the method used to heat up (heat, microwave $\mathrm{e}^{161,168}$ ) the glucose solution and to purify it. Glucose is the basic sugar building block of biomass, which has been extensively studied for synthesizing functional carbon sphere materials by HTC. ${ }^{169}$ Under hydrothermal conditions
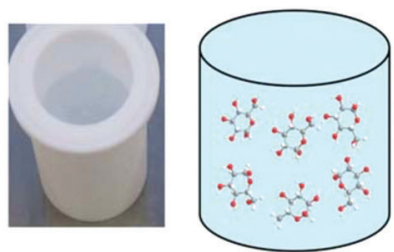

Cyclic Polymerization on Solution Surface

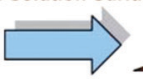

$-\mathrm{H}_{2} \mathrm{O}$

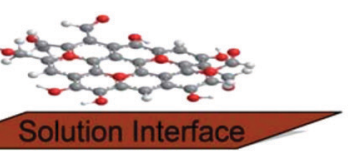

(b) Mono-Layer

(a) Glucose Solution

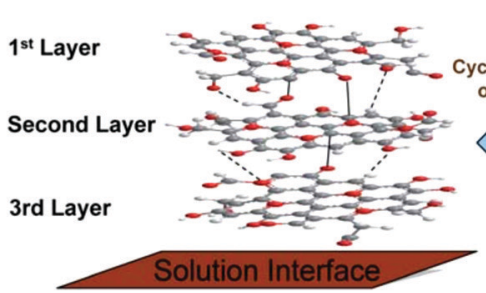

(d) Tri-Layer

yclic Polymerization

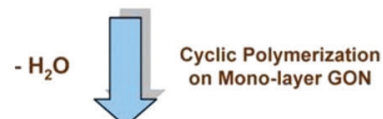
on Bi-layer GON
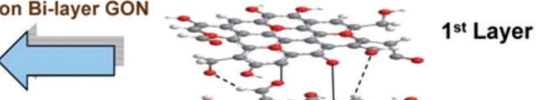

$-\mathrm{H}_{2} \mathrm{O}$

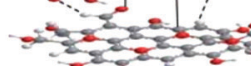

Solution Interface

(c) Bi-Layer

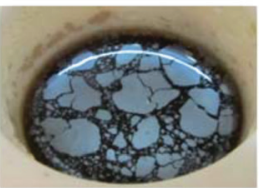

$C=\mathrm{H}$

C $=\mathrm{C}$

$\mathrm{O}=0$

Hift = Glucose

स्रें

(e) Multi-Layer GON

Fig. 27 Schematic illustration of the layer-by-layer bottom-up synthesis mechanism of GON. Under hydrothermal conditions, glucose molecules (a) undergo cyclic polymerization to form monolayer GON (b) which floats on solution due to its hydrophobicity; the second layer of GON may be grown underneath the first GON layer forming a bi-layer GON (c); subsequently, tri-layer (d) and multilayer GONs (e) can be produced. Reproduced from ref. 160 with permission from the Royal Society of Chemistry. 
at relatively low temperatures $\left(<200{ }^{\circ} \mathrm{C}\right)$, glucose molecules underwent a series of dehydration and cross-linking reactions and eventually turned into carbonized spherical colloidal particles dispersed in water, typically insulating nano and micrometer-sized carbon spheres. ${ }^{170}$

\subsection{Thermal decomposition methods for organic matter rich in $\mathrm{C}$}

This section will focus on a class of reactions that uses natural, cheap, highly renewable and abundant organic wastes as a source of carbon. Using low-cost and less sophisticated techniques, oxidized-graphenic materials, monolayer and multilayer graphene oxide, have been synthesized using pyrolysis of bamboo pyroligneous acid, ${ }^{171}$ oxidative chemical exfoliation of rice straw biochar using a modified Hummers' method $^{172}$ and catalytic pyrolysis of sugarcane organic wastes. ${ }^{173}$ By varying the carbonization temperature of bamboo pyroligneous acid (BPA), oxidized-graphenic nanoplatelets (OGNp) can be obtained with oxygen content below $20 \%$. The disordered crystal structure is a mix of $\mathrm{sp}^{2}$ and $\mathrm{sp}^{3}$ characters $\left(\mathrm{sp}^{2}=66 \%\right)$. Nanoplatelets synthesized at higher temperature $(973 \mathrm{~K})$ present lower oxygen content with short-range crystalline order and a carbon environment that is mainly $\mathrm{sp}^{2}(87 \%) .{ }^{171}$ Straw collected from rice biochar was sundried for several days, washed with water to remove dust, dirt and other impurities. Then, it was dried and carbonized. The material obtained was crushed and sieved, and then it was used as a substitute for graphite in the reaction with $\mathrm{KMnO}_{4}$ and $\mathrm{H}_{2} \mathrm{SO}_{4} \cdot{ }^{172}$ Sugarcane bagasse is a type of agricultural waste (agro waste). After its juice was extracted, the remaining fiber was crushed, well ground and mixed with ferrocene. Then it was placed in a crucible and put directly into a muffle furnace at $300{ }^{\circ} \mathrm{C}$ for $10 \mathrm{~min}$ under atmospheric conditions. ${ }^{173}$ The produced materials presented a well-graphitized structure in comparison with those from the commonly used methods. Other structural properties were in terms of morphology, particle size and surface roughness, chemical composition and conductivity very similar to those of conventionally obtained rGO and GO materials.

\section{Post-synthesis treatments}

\subsection{Removal of the impurities from the bulk graphite/graphene} oxide

GO prepared using one of the methods described previously (Section 4) is always recuperated as a crude brownish mixture (Fig. 28a) composed essentially of graphite/graphene oxide sheets and debris of different lateral sizes. Therefore, GO needs to be further purified for adequate characterization (Fig. 28b and d). As prepared, GO contains a large amount of impurities in the bulk such as the unreacted starting materials, hydrophobic carbon materials and carbonaceous materials, ${ }^{27}$ the reagents, the impurities contained in them (metallic elements) ${ }^{44-46}$ and finally, more importantly, $\mathrm{Mn}^{2+}$ when potassium permanganate is used in the Hummers' method. ${ }^{35}$ Those elements have to be washed away appropriately in the purification steps in order to harvest
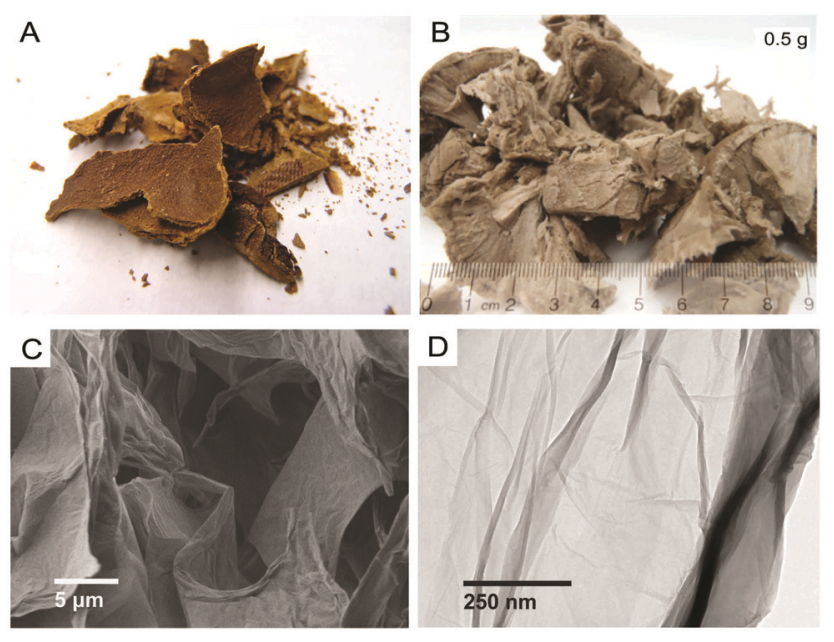

Fig. 28 Optical images of (A) crude GO, (B) GO after centrifugation and lyophilization, (C) SEM and (D) TEM magnification of GO monolayers. Reproduced (adapted) from ref. 174 with permission from the UQÀM (PhD thesis, 2017).

GO with the desired elemental purity for device integration. The common way to get rid of most of the soluble contaminants (metal ions, sulfate, nitrate, phosphate, manganese(Iv), mellitic acid, ${ }^{35}$ etc.) is to wash GO with a diluted solution of hydrogen chloride $(5-10 \% \mathrm{w} / \mathrm{w})$ several times with shaking until the material goes completely into suspension. Between each wash, GO is recuperated either by vacuum filtration or by centrifugation. Then, the residues of $\mathrm{HCl}$ trapped inside GO are removed by washing with a copious amount of Nanopore water. ${ }^{174}$

The remaining salt impurities can be removed by treating with resinous anion and cation exchangers. ${ }^{85}$ Alternatively, GO can be dialyzed for seven (7) days in the dark (with several changes of the dialyzing water) until the solution is free from GO debris particles, sulfate and chloride ions ${ }^{12}$ using typically a polyether sulfone membrane with a pore size of $1.2 \mu \mathrm{m}^{175}$ or a membrane made of regenerative cellulose with a molecular cut-off of $1000^{164}$ to $3000 \mathrm{Da}^{162} \mathrm{GO}$ can be rapidly purified from insoluble, graphitic, hydrophobic and carbonaceous materials by centrifugation at a low speed $(5000 \mathrm{rpm})^{21}$ or by filtration using a metallic drum-sieve and polyester fibre, ${ }^{27}$ glass wool, ceramic paper, woven glass fibre, perlite, or Celite (diatomaceous earths). ${ }^{176}$ Mineral salts or reactive solids (calcium chloride, bentonite clay, kaolinite) are used to flocculate and precipitate GO. Particular solvents or solvent combinations (diethyl ether/ ethanol, ${ }^{27}$ isopropyl alcohol, methanol, acetone) ${ }^{176}$ make GO product flocculate and precipitate, hence more easily filtered, or otherwise separated from its impurities. Furthermore, GO is very hygroscopic and has a high affinity for water. GO imparts a high water uptake capacity of up to 0.58 gram of water per gram of GO. ${ }^{177}$ After its isolation, it needs to be dehydrated at $40{ }^{\circ} \mathrm{C}$, either under vacuum $\left(1 \times 10^{-2} \text { mbar }\right)^{12}$ or over phosphorus pentoxide. ${ }^{85}$

\subsection{Liquid exfoliation techniques}

5.2.1 Acoustic cavitation. Ultrasonic exfoliation of graphite oxide is based on the high shear force induced by acoustic cavitation, 
which is generated by the coupling of powerful ultrasound waves in the liquid. During sonication, very small voids or vacuum bubbles are formed in the liquid. When the vacuum bubbles achieve a size where they cannot absorb more energy, they collapse violently and create a high-pressure acoustic wave. The bubble implosion results in cavitation shear forces and stress waves, extreme temperature and pressure, rapid cooling times, as well as high-speed liquid jets. ${ }^{178}$ Those intense forces affect the graphite stacks, which are delaminated into single- or few-layer graphene oxide sheets. ${ }^{179}$ Long period of ultrasonication could provide the energy for the oxidation of GO sheets. It has been demonstrated that ultrasonication can not only exfoliate and break GO sheets, but also increase the oxidation level of GO by the mechanical shear forces and shockwaves created from the collapse of cavitating bubbles. ${ }^{180}$

The most common way to apply acoustic waves to the GO solution is usually by the immersion of a borosilicate glass beaker containing a suspension of GO in a temperature-controlled sonication water-bath at a frequency of $40 \mathrm{kHz}$ with a power of 40-200 W. Submersible sonication probes such as cell homogenizers with variable power are also very effective in quickly delaminating GO directly in the solution using a series of very short powerful bursts. ${ }^{178}$ This approach is to be used very carefully as these probes have a tendency to heat up the solution, which can be potentially damageable for GO. Also, because the submersible probes are subject to degradation during the highpower bursts, they release ceramic and metal particles in the GO solution, which have to be removed by centrifugation or filtration in an additional step. Briefly, in the laboratory, the crude mixture of GO is usually suspended in Nanopure water $\left(1-5 \mathrm{mg} \mathrm{mL}^{-1}\right)$ using a magnetic stirrer $(24 \mathrm{~h})$, then the suspension is sonicated for a short period of time (30 $\mathrm{min})$. There is a specific sonication time for exfoliation because GO flakes are easily torn during the process. The exposure time of GO solution to ultrasound is critical as they quickly deteriorate the quality of GO resulting in smaller sheets. ${ }^{111}$ Typical size of GO sheets obtained from acoustic cavitation ranges from hundreds of nanometers to a few microns. To avoid overexposure and get more intact larger flakes, one way is to apply in sequence short periods of sonication followed by a separation technique (centrifugation or filtration). This way, GO is removed from the bulk to minimize further deterioration and to better control the desired size of the sheets, while the unexfoliated material is resubmitted to ultrasound treatments. ${ }^{181}$ AFM (atomic force microscopy) is one of the most powerful tools to investigate the exfoliation rate and to evaluate the lateral size and thickness of the graphitic flakes. ${ }^{87}$

5.2.2 Shearing stress. Mechanical exfoliation techniques of graphite are widely used in the production of pristine graphene in very low yield using techniques such as micromechanical cleavage, ball milling, vortex fluidic films, supercritical fluids, pressure driven fluid dynamics, kitchen blenders ${ }^{182}$ and rotostator mixers. ${ }^{183}$ Using Taylor-Couette flow reactors, few-layer graphene can be prepared in bulk quantities for industrially scalable methods. ${ }^{184}$ Shear mixing is already widely used to disperse nanoparticles in liquids and this involves breaking up of nanoparticle agglomerates that are weakly bound. ${ }^{185}$ A high-shear mixer disperses one phase (liquid, solid, gas) into a main continuous phase (liquid), with which it would normally be immiscible. Fluid undergoes shear when one area of fluid travels with a different velocity relative to an adjacent area. Similarly to graphite, GO solution can be exfoliated with controlled dimensions using shear stress in a Couette-Taylor flow reactor ${ }^{186}$ or homogenized using a high-speed blade-impeller (blender), ball-milling $^{187}$ or simply, a magnetic Teflon stirrer or by gentle hand-shaking. ${ }^{115-117}$ Fig. 29 illustrates the three principal methods to exfoliate graphite oxide (sonication, mechanical forces and shearing stress). ${ }^{186}$

5.2.3 Freezing-thawing. To avoid damaging GO sheets using high mechanical and shear stress, an alternative method consists of freezing the GO mixture and subsequent thawing of the resultant solid in a water bath. Hydration of graphite oxide by an excess of water leads to a structural breathing effect for fully hydrated graphite oxide near the water solidification/melting point. Expansion of the hydrated graphite oxide structure due to insertion of additional water occurs upon cooling down from ambient temperature to the point of water media freezing. Cooling down below the point of solidification results in stepwise contraction of the graphite oxide interlayer distance by $25 \%$ due to partial withdrawal of water from the hydrated structure. Heating back from $230 \mathrm{~K}$ to ambient temperature results in graphite oxide structure expansion due absorption of water from medium, thus making a reversible cycle. ${ }^{188}$ This approach is used to exfoliate the layered structure of graphite oxide into GO that is dispersed in water (Fig. 30). After repeating the freeze-thaw sequence $(6 \times)$, relatively large GO sheets are obtained with $80 \%$ yield with minimal fragmentation. Furthermore, directional freezing using liquid nitrogen of the resultant solution reassembles the nanosheets into a threedimensional lightweight monolith that has a honeycomb-like morphology. ${ }^{189}$

5.2.4 Micro bubbling. An alternative method for producing uniform dispersion of large surface-area GO sheets is accomplished by capturing $\mathrm{CO}_{2}$ gas pre-intercalated with water molecules in the interlayers of GO. GO-powder swollen with water $(40 \% \mathrm{w} / \mathrm{w})$ is pressurized with $\mathrm{CO}_{2}$ gas (5-20 bar), and then frozen. At $-30{ }^{\circ} \mathrm{C}$, the intercalated $\mathrm{CO}_{2}$ gas molecules are trapped between the basal plane of GO and the ice. Both $\mathrm{CO}_{2}$ and ice structure play very important roles, not only by reducing the van der Waals interaction and $\pi-\pi$ stacking between GO sheets, but also by applying pressure against the sheets. This force is provided by the pressure of $\mathrm{CO}_{2}$ molecules spontaneously ejected from the GO interlayer as surrounding ice rapidly melts upon warming up in a solvent, and this allows the GO sheets to be exfoliated without intercalant-removal or sonication processes. ${ }^{190}$ The gas bubbling exfoliation process can also be achieved by adapting the Hummers' method in a way that the gas released (oxygen) from the quenching of $\mathrm{KMnO}_{4}$ with $\mathrm{H}_{2} \mathrm{O}_{2}$, at the final stage of the reaction, produces enough localized pressure to delaminate the graphite stacks oxidized at the edge. After edge oxidation, the bubbling exfoliation immediately and spontaneously takes place when the precursor is mixed with a solution of $\mathrm{NH}_{3}$ and $\mathrm{H}_{2} \mathrm{O}_{2}$. A lot of bubbles appear on the graphite sheets, and as a result 


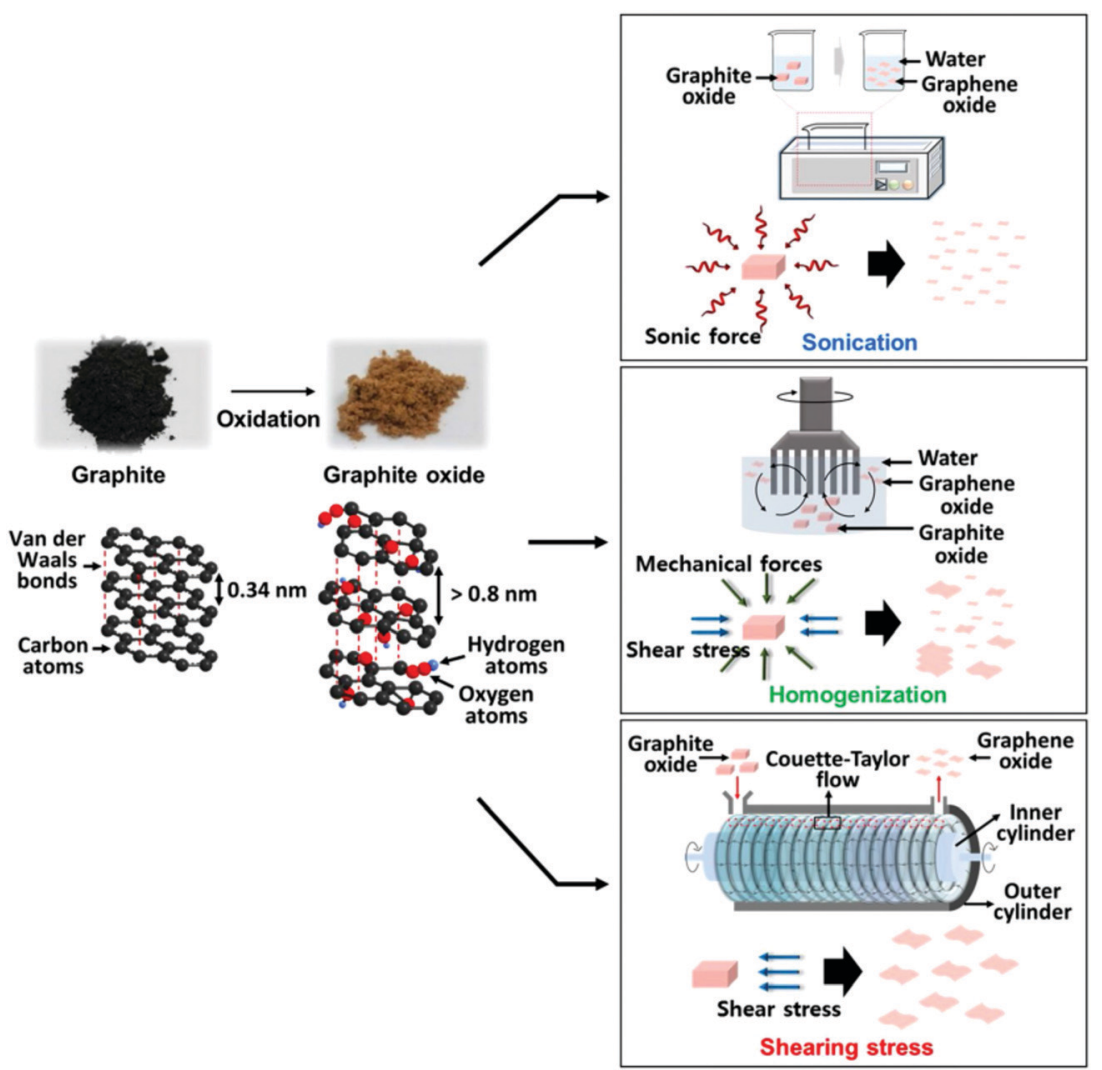

Fig. 29 Schematic of various exfoliation methods of graphite oxide (sonication, homogenization, and shearing stress). Reproduced from ref. 186 with permission from the NPG. http://creativecommons.org/licenses/by/4.0/.

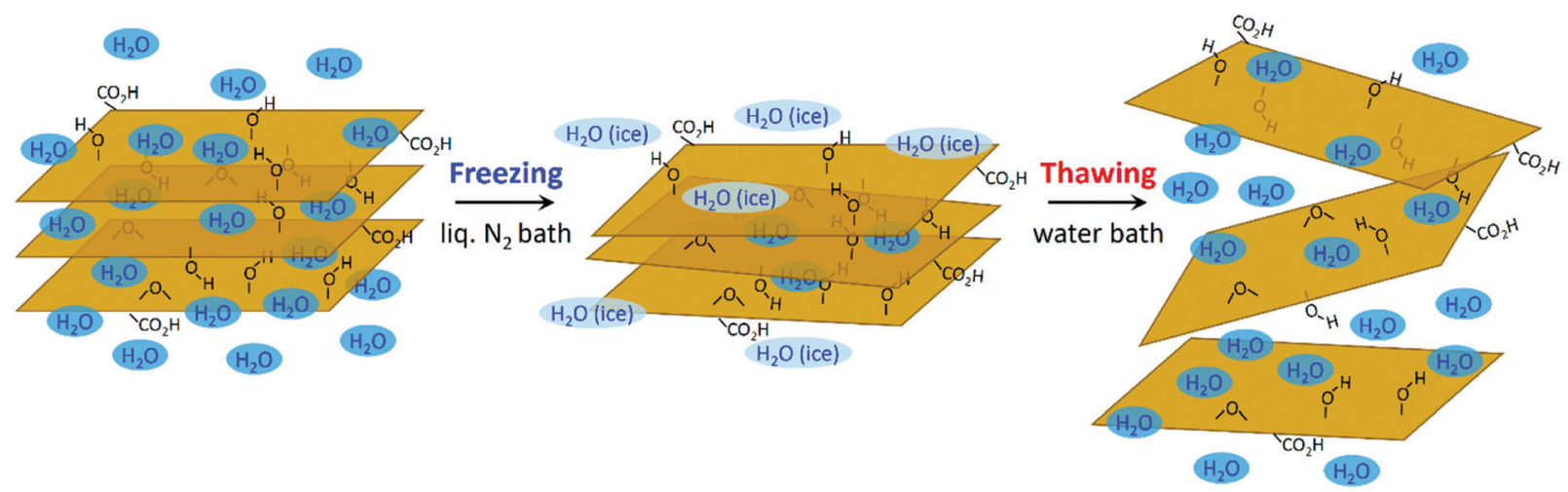

Fig. 30 Exfoliation of graphite oxide via rapid freezing of hydrated graphite oxide flakes in water and subsequent thawing of the resultant solid. Rapid freezing and subsequent thawing cycles are hypothesized to cause fast structural breathing (rapid changes in the interlayer spacing between layers), which leads to the efficient exfoliation of graphite oxide layers and the formation of dispersed nanosheets. Reprinted with permission from (Chem. Mater., 2014, 26, 3334-3339). Copyright (2014) American Chemical Society.

the sheets separate and transform into water-dispersible graphene materials. ${ }^{191}$

\subsection{Fractionation of GO sheets by size}

5.3.1 Centrifugation. Centrifugal separation is a simple method used for size sorting of nanomaterials distributed in liquid medium.

It is a technique that relies on the fact that the sedimentation rate of a material is dependent on its shape, size, and buoyant density, as well as the density and viscosity of the centrifugal medium and the rotor speed.

As the rotor of centrifuge spins with a definite angular velocity, an apparent centrifugal force acts on the suspension and pushes radially outwards towards the side of the tube in the meantime. There is a correlation between the size and density of a particle and the rate at which the particle separates from a colloidal mixture, when the only force applied is that of gravity (sedimentation). The larger the size and the larger the 
density of the particles, the faster they separate from the mixture. By applying a larger effective gravitational force to the mixture, like a centrifuge does, the separation of the particles is accelerated. ${ }^{192}$

Gradient differential centrifugation (GDC). GDC is commonly performed using distilled water as the dispersive medium. Under centrifugation force, large GO sheets precipitate at a lower centrifugation speed (5000 rpm) and are recuperated from the solution, while smaller sheets remain in the supernatant and need stepwise speed increments (gradient differential) up to $25000 \mathrm{rpm}$ to settle in the tube with size differentiation (Fig. 31). ${ }^{174,193}$ Alternatively, the initial dispersion is centrifuged at a high centrifugation rate, separating small flakes in the supernatant from large flakes in the sediment. Redispersion of the sediment, followed by successive centrifugation, separation and redispersion cycles can be used to separate the flakes by size so long as the centrifugation rate is decreased with each cycle. ${ }^{194,195}$

Density gradient centrifugation (DGC). $\mathrm{DGC}^{196}$ is a variation where the components of a sample are separated on the basis of their density, which is theoretically independent of lateral area. After being centrifuged in a water-glycerol mixture, ${ }^{197}$ in sucrose, ${ }^{198}$ or in iodixanol solutions with density higher than water, GO will locate at a different height within the tube corresponding to its buoyant density (Fig. 32). ${ }^{199}$ Centrifugation of such density gradients causes the GO sheets to move to their isopycnic points - locations where the density of the medium matches the buoyant density of the GO. Compared to differential centrifugation, DGC requires significantly longer times to afford enough time to the slowest particles to reach their isopycnic points. ${ }^{192}$

5.3.2 Filtration on membranes with well-defined pore size. Polycarbonate track-etched membranes with uniform pore size are used as filters to separate GO sheets into different size fractions. In this technique, an aqueous dispersion of GO is filtrated through a series of filter membranes with a given pore size. Theoretically, GO sheets with small lateral dimensions are expected to pass through the pores, while the relatively large
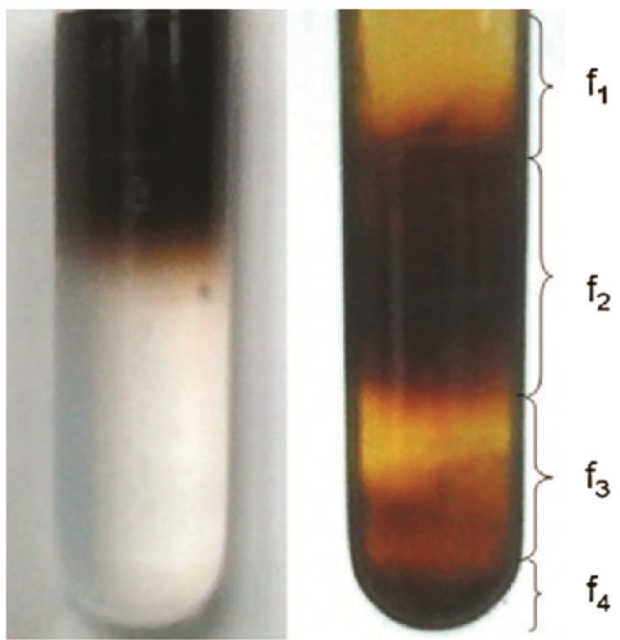

Fig. 32 Photograph of a centrifuge tube (left) before and (right) after density gradient centrifugation (DGC). Reproduced from ref. 199 with permission from the Elsevier Science.

sheets are blocked. Practically, the concentration of GO dispersion needs to be controlled to avoid the aggregation of GO sheets. At relatively high concentrations, GO sheets behave like large aggregated particles rather than individual sheets, decreasing the efficiency of size fractionation. The exclusive diffusion of GO sheets through the filter membrane becomes extremely slow at concentrations higher than $2.0 \mathrm{mg} \mathrm{mL}^{-1}$. Therefore, the concentration of GO dispersion should be controlled to be lower than this value during the whole process of size fractionation. The effectiveness of size fractionation using membrane filtration is illustrated in Fig. $33 .^{200}$

5.3.3 Directional freezing. Directional freezing of the precursor GO hydrogels under controlled conditions leads to the formation of ice rods in the micrometer range within the hydrogels, which act as a template for forming straight macropores interconnected with micro- and/or mesopores present within the honeycomb walls. The formation of the pore structures is

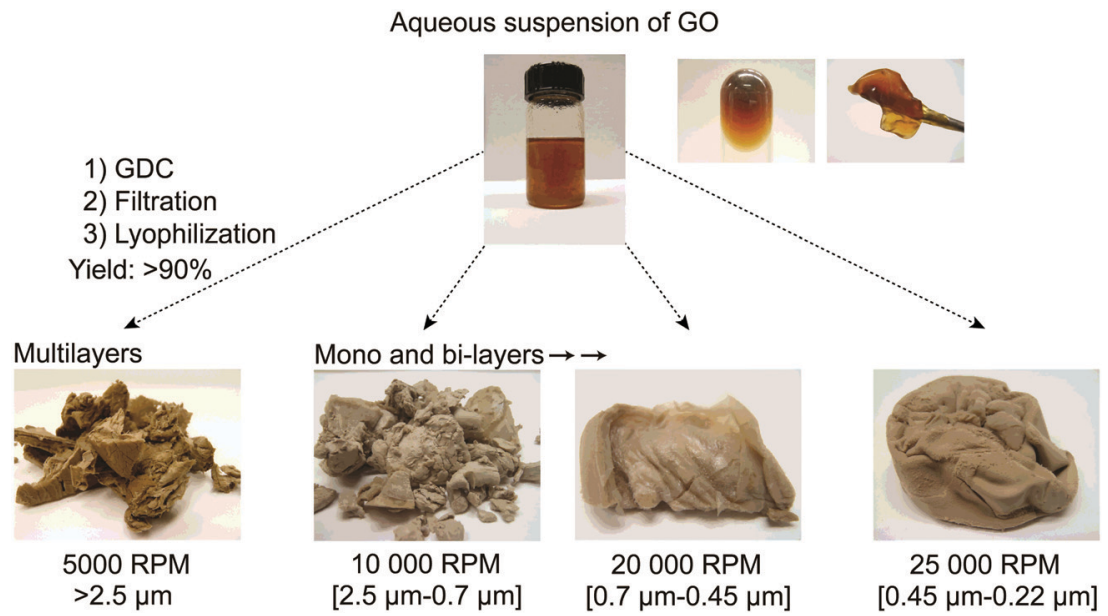

Fig. 31 Fraction of GO obtained after gradient differential centrifugation (GDC) and membrane filtration of an aqueous suspension of GO. Reproduced (adapted) from ref. 174 with permission from the UQȦM (PhD thesis, 2017). 

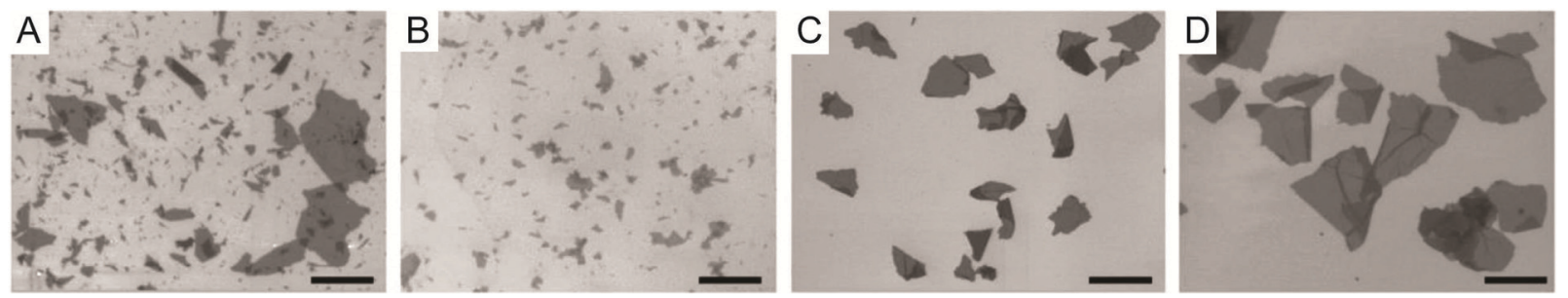

Fig. 33 Typical SEM image of (A) conventional GO, (B) small GO sheets, (C) medium GO sheets and (D) large GO sheets; Scale bar: $20 \mu \mathrm{m}$. Reproduced (adapted) from ref. 200 with permission from Wiley- $\mathrm{VCH}$.

believed to result from the self-assembly of large 2D GO sheets during the freezing process. ${ }^{189}$ During controlled directional freezing (top-down), size fractionation of GO nanosheets with a narrow size distribution can be obtained by controlling the growth rate of the freezing front. This interesting phenomenon can be explained by the adsorption of GO nanosheets on the ice crystal surface in combination with the stratification of GO nanosheets at the ice growth front. When the growth rate of the freezing front is appropriate, the synergetic effect of the growing ice on GO nanosheets, the Brownian motion of GO nanosheets, and the cross-interaction of GO nanosheets of different sizes lead to the stratification of GO nanosheets at the ice growth front. As such, GO nanosheets with a narrow size distribution are adsorbed on the ice crystal surface because of the formation of hydrogen bonds between GO nanosheets and ice crystals. Therefore, the larger GO sheets remain in the unfrozen residual liquid (Fig. 34). ${ }^{201}$

5.3.4 Partition in water-oil emulsion. Using the formation of GO stabilized oil-in-water emulsions with chloroform, heptane and benzene, GO can be separated into an emulsion fraction (GOe) and a water fraction (GOw). The trend shows that the emulsion fraction always prefers the less oxidized, or more graphitic, population of the available GO in the system, while on the other hand, more oxidized GO sheets have higher affinity for the water phase. Repeated water extractions are shown to increase the enrichment of GO in either higher or lower levels of oxidized material of both phases. The fractionation of GO results from the less oxidized material's ability to stabilize oil-in-water emulsions preferentially to more oxidized material and allows for the preparation of narrowly defined samples. For a typical fractionation procedure, GO $\left(4 \mathrm{mg} \mathrm{mL}^{-1}\right)$ is added to DI water $(20 \mathrm{~mL})$, then bath-sonicated for $1 \mathrm{~min}$. After sonication, chloroform $(5 \mathrm{~mL})$ is added to the suspension and the mixture is homogenised leading to the formation of a stable emulsion (Fig. 35). Then, the layers are separated using conventional techniques (pipette, separatory funnel, etc.) For multiple extractions, water is re-added to the emulsion and the mixture is homogenized again. ${ }^{202}$

5.3.5 Circular flow. With the help of hydrogen gas $\left(\mathrm{H}_{2}\right)$ bubbles generated from a metallic replacement reaction, continuous turbulence forms a circular flow in a special tubular container making it possible to fractionate GO sheets by size (Fig. 36). By using this process, crude GO sheets can be separated into three size ranges: large $(>20 \mu \mathrm{m})$, medium $(2-20 \mu \mathrm{m})$ and small $(<2 \mu \mathrm{m})$. An advantage of this approach is that during the (a)

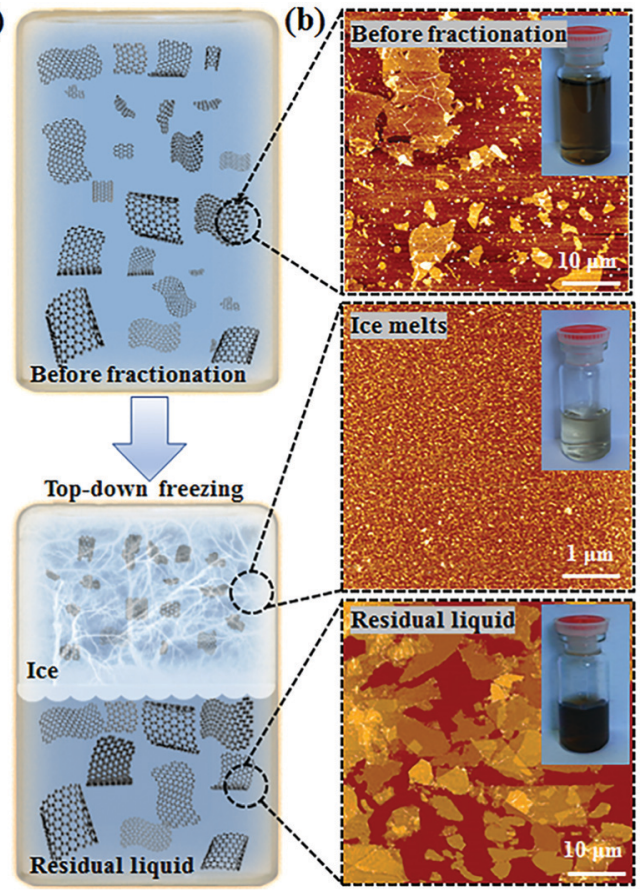

Fig. 34 (a) Illustration of the size fractionation of GO via the controlled directional freezing. The GO aqueous dispersion was frozen from the top at a controlled freezing rate. GO nanosheets with smaller sizes were obtained from the ice melt, and larger sheets were left in the residual liquid. (b) AFM images of the GO nanosheets obtained from the GO dispersion before fractionation, the ice melt, and the residual liquid (from top to bottom). Insets are the corresponding digital photos. Reprinted with permission from (J. Am. Chem. Soc., 2017, 139, 12517-12523). Copyright (2017) American Chemical Society.

separation process residual graphite oxide can be simultaneously removed, gradually precipitated into the cone groove at the bottom of the tube by gravity and collected at the end of the separation. As shown in Fig. $36, \mathrm{H}_{2}$ bubbles are mainly generated from the metallic replacement reaction around the cathode, moving near the side wall of the tube and causing continuous turbulence in the dispersion, which leads to a circular flow in the device. Then, the circular flow would prevent the sheets in the flow from aggregation especially in the middle part of the dispersion. Small GO sheets have a higher charge-to-mass ratio than large ones due to high-density carboxyl groups and phenol groups. Thus, SGO sheets, benefitting from the circular flow, are attracted to the anode at the top of the device because of many negatively charged ionized groups. Medium GO sheets have to stay in the circular flow because of low charge-mass 


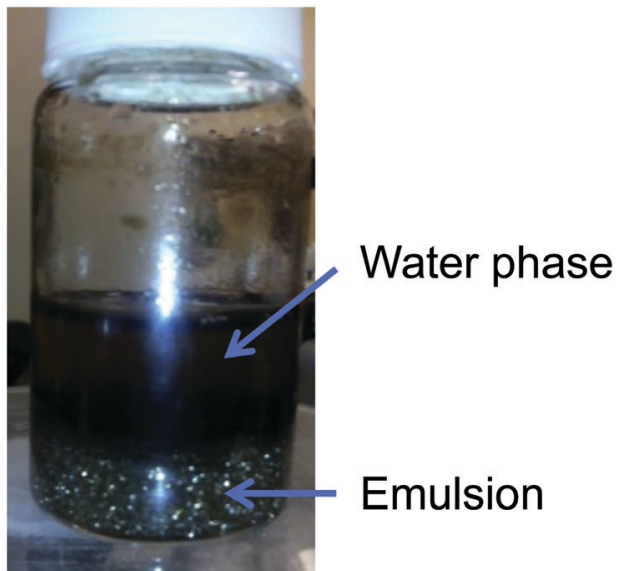

Fig. 35 Image of fractionated GO. The upper phase contains GO suspended in water and the lower region contains GO at the interface of a chloroform-in-water emulsion. Reproduced from ref. 202 with permission from the Elsevier Science.

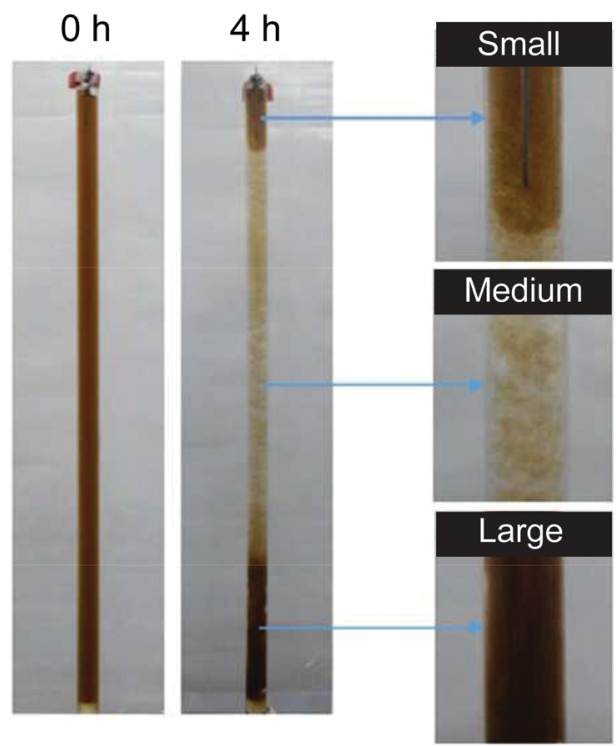

Fig. 36 Digital images of GO separation results after $4 \mathrm{~h}$ using the circular flow technique. Reproduced (adapted) from ref. 203 with permission from the Royal Society of Chemistry.

ratio without interrupting the larger sheets to aggregate at the bottom and the impurities at the groove of the device. Then, LGO sheets are aggregated first near the bottom of the tube in the edge-to-edge/face mode and most of the larger sheets precipitate in a relatively short time period at the beginning of the fractionation. After 4 hours of separation, the fractions were collected using a syringe pump inserted through the cathode tube. ${ }^{203}$

\section{Conclusion and outlook}

The amount of scientific research devoted to the preparation of graphite oxide and graphene oxide is tremendous and new approaches are constantly being explored. GO is composed of carbon, hydrogen and oxygen atoms, and independently of the way chosen to make it, its structure and reactivity are regulated by the concepts and principles of organic chemistry. At the current state of graphene research, the synthesis of GO via the Hummers' and the electrochemical methods for graphite are the most promising approach towards large scale production of graphene materials. Although the resulting GO is imperfect as compared to graphene obtained from microcleavage, it is a useful platform for the fabrication of functionalized graphene-based devices that can potentially improve mechanical, thermal and electrical properties. GO can be shaped into paper-like membranes using filtration or casting techniques, scrolls by shear stress, tubes, monolithic foams and sponges by freeze-casting freeze-drying techniques and fibers using spinning methods. GO has potential use in transparent conductive layers, nanoelectronics, solar cells, chemical and biosensors, electrochemistry, brain implants, catalysis, coatings, paints/3D inks, polymer composites, textiles, concrete and construction, membranes for audio transducers, environmental remediation, drug delivery and energy storage (batteries). A cheaper cost of production and the ability to fine-tune the properties of GO could accelerate the integration of these start-up technologies in our daily life. While GO and related materials have been known for more than one century, they continue to attract the curiosity and remain the principal central point of interest of many fundamental and applied studies in nanotechnology.

In summary, GO has its own history that can be traced back to 1859 , independently of its relationship with graphene, which has attracted considerable attention since 2010 when Geim and Novoselov won a Nobel Prize for discovering graphene from graphite. In this review, we provided a historical perspective of its structure and preparation. So far, the GO materials have contained a significant amount of oxygen and, possibly, significant numbers of defects. CVD repairing of GO and rGO sheets have produced enhanced results and finding routes for complete restoration of the $\mathrm{sp}^{2}$ carbon network is of current interest. For the last decade (2007 to 2016), the annual global emission of $\mathrm{CO}_{2}$ was estimated to be 9.4 billion metric tons, ${ }^{204}$ which is more than 12 times greater than the world's inferred resources exceeding 800 million tons of recoverable graphite. ${ }^{205}$ If GO could be produced on a large scale from various precursors, including organic wastes or environmental carbon dioxide, the supply of GO might be enormously increased. More importantly, rational synthetic modifications could potentially provide novel procedures or even novel graphene-based materials for future practical applications, and at the same time, this would have a positive impact on the climate change for example. The progress of commercial applications of graphene is still at its beginning. At this moment, it is critical to highlight the most appropriate method for the preparation of graphene oxide to ensure its quality and supply. We hope that this review, multidisciplinary in nature, will be valuable and beneficial to find new ways to make GO and related materials on a global scale.

\section{Authors contributions}

P. P. B. performed the literature search and wrote the manuscript including the preparation of figures and tables. All authors reviewed the manuscript prior to submission. 


\section{Funding}

P. P. B. is grateful to FR-QNT (Fonds de Recherche Québécois Nature et technologies) for continuous postgraduate research funding (2013-2017: PhD (UQÀM) and PDF (UofToronto/ McGill)). This work is financed in part by the Natural Sciences \& Engineering Research Councils (NSERC) of Canada and NanoQAM.

\section{Conflicts of interest}

There are no conflicts to declare.

\section{Acknowledgements}

We thank all M. Siaj's laboratory members, interns and collaborators who have worked on the synthesis of graphene oxide and shared their passion for chemistry of materials.

\section{References}

1 S. Eigler and A. M. Dimiev, Graphene Oxide: Fundamentals and Applications, John Wiley and Sons, 1st edn, 2017.

2 W. Gao, Graphene Oxide: Reduction Recipes, Spectroscopy, and Applications, Springer Springer Cham, Heidelberg New York Dordrecht London, 2015.

3 J. Zhao, et al., Graphene Oxide: Physics and Applications, Heidelberg New York Dordrecht London, 2014.

4 S. Park and R. S. Ruoff, Nat. Nanotechnol., 2009, 4, 217-224.

5 D. Chen, et al., Chem. Rev., 2012, 112, 6027-6053.

6 D. R. Dreyer, et al., Chem. Soc. Rev., 2014, 43, 5288-5301.

7 D. R. Dreyer, et al., Chem. Soc. Rev., 2010, 39, 228-240.

8 S. Eigler and A. Hirsch, Angew. Chem., Int. Ed., 2014, 53, 7720-7738.

9 A. Lerf, et al., J. Phys. Chem. B, 1998, 102, 4477-4482.

10 A. Lerf, et al., Solid State Ionics, 1997, 103, 857-862.

11 H. He, et al., Chem. Phys. Lett., 1998, 287, 53-56.

12 H. He, et al., J. Phys. Chem., 1996, 3654, 19954-19958.

13 W. Cai, et al., Science, 2008, 321, 1815-1818.

14 T. Szabó, et al., Chem. Mater., 2006, 18, 2740-2749.

15 T. Szabó, et al., Carbon, 2005, 43, 3186-3189.

16 W. Gao, et al., Nat. Chem., 2009, 1, 403-408.

17 B. K. Erickson, et al., Adv. Mater., 2010, 22, 4467-4472.

18 T. Dimiev, et al., J. Am. Chem. Soc., 2012, 134, 2815-2822. 19 S. Eigler, et al., Chem. - Eur. J., 2013, 19, 9490-9496.

20 L. Yang, et al., Angew. Chem., Int. Ed., 2014, 53, 10109-10113.

21 P. P. Brisebois, et al., Chem. - Eur. J., 2016, 22, 5849-5852.

22 W. R. Collins, et al., Angew. Chem., Int. Ed., 2011, 50, 8848-8852.

23 C. K. Chua and M. Pumera, Chem. Soc. Rev., 2014, 43, 291-312.

24 H. L. Poh, et al., Nanoscale, 2012, 4, 3515-3522.

25 C. K. Chua, et al., Chem. - Eur. J., 2012, 18, 13453-13459.

26 Z. Sofer, et al., ACS Nano, 2015, 9, 5478-5485.

27 D. C. Marcano, et al., ACS Nano, 2010, 4, 4806-4814.

28 A. Y. S. Eng, et al., Chem. - Eur. J., 2013, 19, 12673-12683.
29 S. Park, et al., Nano Lett., 2009, 9, 1593-1597.

30 N. Morimoto, et al., Sci. Rep., 2016, 6, 21715.

31 E. C. Salas, et al., ACS Nano, 2010, 4, 4852-4856.

32 O. O. Ekiz, et al., ACS Nano, 2011, 5, 2475-2482.

33 D. Voiry, et al., Science, 2016, 361, 1413-1416.

34 P. Šimek, et al., Adv. Funct. Mater., 2014, 24, 4878-4885.

35 R. K. Singh, et al., RSC Adv., 2016, 6, 64993-65011.

36 A. T. Smith, et al., Nano Mater. Sci., 2019, 1, 31-47.

37 O. Jankovský, et al., Appl. Mater. Today, 2016, 4, 45-53.

38 L. Wang, et al., J. Mater. Chem. C, 2014, 2, 2887-2893.

39 H. L. Poh, et al., ACS Nano, 2013, 76, 5262-5272.

40 X. Li, et al., J. Mater. Chem. C, 2013, 1, 7308-7313.

41 L. M. Rivera, et al., Catalysts, 2017, 7, 278.

42 L. Wang, et al., Angew. Chem., Int. Ed., 2013, 52, 13818-13821.

43 Y. Shi and L.-J. Li, J. Mater. Chem., 2011, 21, 3277-3279.

44 C. H. A. Wong, et al., Proc. Natl. Acad. Sci. U. S. A., 2014, 111, 13774-13779.

45 A. Ambrosi, et al., Proc. Natl. Acad. Sci. U. S. A., 2012, 109, 12899-12904.

46 A. Ambrosi, et al., Angew. Chem., Int. Ed., 2012, 51, 500-503. 47 L. Wang, et al., Nanoscale, 2019, 11, 11083-11085.

48 J. Zhang, et al., Chem. Commun., 2010, 46, 1112-1114.

49 S. H. Dave, et al., ACS Nano, 2016, 10, 7515-7522.

50 S. Park, et al., ACS Nano, 2008, 2, 572-578.

51 D. Li, et al., Nat. Nanotechnol., 2008, 3, 101-105.

52 J. Ma, et al., Membranes, 2017, 7, 52-81.

53 S. Mura, et al., ACS Appl. Nano. Mater., 2018, 1, 6724-6732.

54 X. Yang, et al., RSC Adv., 2012, 2, 8821.

55 K. H. Thebo, et al., J. Mater. Sci. Technol., 2018, 34, 1481-1486.

56 D. A. Dikin, et al., Nature, 2007, 448, 457-460.

57 S. J. Kim, et al., Sci. Rep., 2018, 8, 1959.

58 R. Jalili, et al., ACS Nano, 2013, 7, 3981-3990.

59 M. Khan, et al., J. Mater. Chem. A, 2015, 3, 18753-18808.

60 R. K. Singh, et al., RSC Adv., 2016, 6, 64993-65011.

61 Y. Wang, et al., J. Phys. Chem. C, 2011, 115, 23192-23197.

62 X. Wang, et al., J. Am. Chem. Soc., 2011, 133, 6338-6342.

63 W. Zhang, et al., RSC Adv., 2015, 5, 146-152.

64 B. Konkena and S. Vasudevan, J. Phys. Chem. Lett., 2012, 3, 867-872.

65 J. Zhao, et al., Anal. Chem., 2011, 83, 9100-9106.

66 V. Nicolosi, et al., Science, 2013, 340, 1226419.

67 Y. Zhu, et al., ACS Nano, 2010, 4, 1227-1233.

68 J. I. Paredes, et al., Langmuir, 2008, 24, 10560-10564.

69 M. Pumera and Z. Sofer, Chem. Soc. Rev., 2017, 46, 4450-4463.

70 D. Cheng, et al., J. Electrochem. Soc., 2017, 164, H345-H351.

71 Z. Miao, et al., RSC Adv., 2016, 6, 58561-58565.

72 J. Chen, et al., Chem. Sci., 2016, 7, 1874-1881.

73 K. Klímová, et al., J. Phys. Chem. C, 2016, 120, 24203-24212.

74 Q. Yang, et al., Ind. Eng. Chem. Res., 2012, 51, 310-317.

75 A. Bakandritsos, et al., ACS Nano, 2017, 11, 2982-2991.

76 I.-Y. Jeon, et al., Proc. Natl. Acad. Sci. U. S. A., 2012, 109, 5588-5593.

77 H. L. Poh, et al., Chem. - Eur. J., 2015, 21, 8130-8136.

78 U. Rajeena, et al., New J. Chem., 2018, 42, 9658-9665.

79 J. Sun, et al., ACS Appl. Mater. Interfaces, 2016, 8, 10226-10233.

80 L. Yan, et al., J. Mater. Chem., 2012, 22, 8367-8371. 
81 B. Gupta, et al., Sci. Rep., 2017, 7, 45030.

82 B. C. Brodie, Philos. Trans. R. Soc. London, 1859, 149, 249-259. 83 L. Staudenmaier, Ber. Dtsch. Chem. Ges., 1898, 31, 1481-1487.

84 V. L. Hofmann and E. Konig, Z. Anorg. Allg. Chem., 1937, 31, 311-336.

85 W. S. Hummers and R. E. Offeman, J. Am. Chem. Soc., 1958, 80, 1339.

86 A. M. Dimiev and J. M. Tour, ACS Nano, 2014, 8, 3060-3068. 87 G. Shao and Y. Lu, et al., J. Mater. Sci., 2012, 47, 4400-4409. 88 S. Pan and I. A. Aksay, ACS Nano, 2011, 5, 4073-4083. 89 J. H. Kang, et al., Chem. Mater., 2016, 28, 756-764.

90 D. R. Chowdhury, et al., RSC Adv., 2014, 4, 15138-15145. 91 J. Chen, et al., Carbon, 2013, 64, 225-229.

92 S. Chen, et al., Carbon, 2015, 81, 826-834.

93 M. Zhang, et al., Adv. Mater., 2015, 27, 6708-6713.

94 N. I. Kovtyukhova, et al., Chem. Mater., 1999, 11, 771-778. 95 S. Eigler, Chem. Commun., 2015, 51, 3162-3165.

96 H. Yu, et al., Sci. Rep., 2016, 6, 36143.

97 A. Esmaeili and M. H. Entezari, J. Colloid Interface Sci., 2014, 432, 19-25.

98 C. Y. Su, et al., Chem. Mater., 2009, 21, 5674-5680.

99 X. Wang, et al., RSC Adv., 2014, 4, 60102.

100 O. Y. Posudievsky, et al., J. Mater. Chem. A, 2013, 1, 6658.

101 D. C. Marcano, et al., ACS Nano, 2018, 12, 2078.

102 P. Ranjan, et al., Sci. Rep., 2018, 8, 12007.

103 D. Luo, et al., Mater. Today Phys., 2019, 9, 100097.

104 S. Eigler, et al., Adv. Mater., 2013, 25, 3583-3587.

105 H. Chen, et al., Chem. Sci., 2019, 10, 1244-1253.

106 J. Qin, et al., J. Mater. Chem. A, 2019, 7, 9646-9655.

107 G. Wang, et al., Appl. Phys. Lett., 2011, 99, 53114.

108 J. Chen, et al., Carbon, 2016, 110, 34-40.

109 J. P. Zhao, et al., ACS Nano, 2010, 4, 5245-5252.

110 L. Zhang, et al., Carbon, 2009, 47, 3365-3380.

111 C. Botas, et al., Carbon, 2013, 63, 562-592.

112 D. Kang and H. S. Shin, Carbon Lett., 2012, 13, 39-43.

113 J. Guerrero-Contreras and F. Caballero-Briones, Mater. Chem. Phys., 2015, 153, 209-220.

114 K. Krishnamoorthy, et al., Carbon, 2013, 53, 38-49.

115 L. Dong, et al., Chem. Mater., 2017, 29, 564-572.

116 L. Dong, et al., Chem. Soc. Rev., 2017, 46, 7306-7316.

117 A. T. Smith, et al., Nano Mater. Sci., 2019, 1, 31-47.

118 T. Ishikawa, et al., Composites of oxidized graphite material and expanded graphite material, Nippon Carbon Co., USPTO, USA, 1978.

119 M. Wojtoniszak and E. Mijowska, J. Nanopart. Res., 2012, 14, 1248-1255.

120 L. Peng, et al., Nat. Commun., 2015, 6, 5716-5725.

121 Z. Sofer, et al., Angew. Chem., Int. Ed., 2016, 55, 11965-11969.

122 C. Yu, et al., Sci. Rep., 2016, 6, 17071.

123 Y. Shin, et al., Nanoscale, 2015, 7, 5633-5637.

124 J. Shen, et al., Chem. Mater., 2009, 21, 3514-3520.

125 P. Yu, et al., Chem. Mater., 2016, 28, 8429-8438.

126 S. Pei, et al., Nat. Commun., 2018, 9, 145.

127 S. E. Lowe, et al., ACS Appl. Nano Mater., 2019, 2, 867-878. 128 A. Ambrosi and M. Pumera, Chem. - Eur. J., 2016, 22, 153-159. 129 Z. Tian, et al., Carbon, 2017, 112, 185-191.
130 A. Ambrosi, et al., Chem. Rev., 2014, 114, 7150-7188.

131 K. Kakaei and K. Hasanpour, J. Mater. Chem. A, 2014, 2, 15428.

132 X. You, et al., J. Nanosci. Nanotechnol., 2011, 11, 5965-5968.

133 H. Imran, et al., RSC Adv., 2015, 5, 63513-63520.

134 N. E. Sorokina, et al., Inorg. Mater., 2001, 37, 360-365.

135 B. Gurzȩda, et al., Carbon, 2016, 100, 540-545.

136 L. Lu, et al., Sci. Rep., 2015, 5, 16242.

137 N. Kumar and V. C. Srivastava, ACS Omega, 2018, 3, 10233-10242.

138 O. O. Ekiz, et al., ACS Nano, 2011, 5, 2475-2482.

139 C. Zhu, et al., RSC Adv., 2014, 4, 55044-55047.

140 C. Zhu, et al., Nanoscale, 2013, 5, 8982-8985.

141 L. Liu, et al., Nanoscale, 2015, 7, 13619-13628.

142 Y. Q. Lu, et al., Green Chem., 2017, 19, 900.

143 M. Lee, et al., Sci. Rep., 2015, 5, 11707.

144 M. Zhang, et al., J. Mater. Chem. B, 2016, 4, 121-127. 145 N. Karoukis, et al., Chem. Rev., 2016, 116, 4850-4883. 146 D. V. Kosynkin, et al., Nature, 2009, 458, 872-876. 147 L. Sun and B. Fugetsu, Mater. Lett., 2013, 109, 207-210. 148 Z. Luo, et al., J. Am. Chem. Soc., 2009, 131, 898-899. 149 T. Liu, et al., Carbon, 2017, 119, 544-547.

150 S. Zhao, et al., Nanotechnology, 2012, 23, 355703.

151 A. Nourbakhsh, et al., Nanotechnology, 2010, 21, 435203.

152 L. Weng, et al., Appl. Phys. Lett., 2008, 93, 9-11.

153 H. C. Lee, et al., RSC Adv., 2017, 7, 15644-15693.

154 W. Choi, et al., Crit. Rev. Solid State Mater. Sci., 2010, 35, 52-71.

155 Y. Liu and Y. Chen, J. Appl. Phys., 2016, 119, 103301, DOI: 10.1063/1.4942999.

156 L. Cardenas, et al., Nanoscale, 2014, 6, 8664-8670.

157 J. Huang, et al., Nanoscale, 2013, 5, 2945-2951.

158 V. López, et al., Adv. Mater., 2009, 21, 4683-4686.

159 S. Grimm, et al., J. Phys. Chem. C, 2016, 120, 3036-3041.

160 L. Tang, et al., J. Mater. Chem., 2012, 22, 5676-5683.

161 L. Tang, et al., Part. Part. Syst. Charact., 2013, 30, 523-531.

162 T. V. Tam, et al., J. Mater. Chem. A, 2017, 5, 10537.

163 L. Tang, et al., J. Mater. Chem. C, 2013, 1, 4908-4915.

164 L. Tang, et al., ACS Nano, 2014, 8, 6312-6320.

165 F. Pan, et al., ACS Appl. Mater. Interfaces, 2013, 5, 11108-11114.

166 L. Yang, et al., RSC Adv., 2018, 8, 5902-5911.

167 X. Li, et al., Nanoscale, 2014, 6, 5323-5328.

168 B. Zheng, et al., Nanophotonics, 2017, 6, 259-267.

169 N. Papaioannou, et al., Sci. Rep., 2018, 8, 6559.

170 D. Krishnan, et al., ACS Nano, 2014, 8, 449-457.

171 J. J. Prías-Barragán, et al., Phys. Status Solidi A, 2016, 213, 85-90.

172 S. Goswami, et al., Process Saf. Environ. Prot., 2017, 106, 163-172.

173 T. Somanathan, et al., Nanomaterials, 2015, 5, 826-834.

174 P. P. Brisebois, Thesis, Montreal (Quebec, Canada), Université du Québec à Montréal, 2017, PhD Chemistry, http://archipel.uqam.ca/id/eprint/9864.

175 F. J. Tölle, et al., Carbon, 2014, 75, 432-442.

176 G. Ceriotti, et al., RSC Adv., 2015, 5, 50365-50371.

177 B. Lian, et al., Chem. Sci., 2018, 9, 5106-5111.

178 J. T. Han, et al., Sci. Rep., 2014, 4, 5133. 
179 C. Cai, et al., J. Exp. Nanosci., 2017, 12, 247-262. 180 X. Qi, et al., J. Mater. Sci., 2014, 49, 1785-1793. 181 U. Sierra, et al., Carbon, 2014, 80, 830-832. 182 J. M. Tour, Nat. Mater., 2014, 13, 545-546. 183 M. Yi and Z. Sheng, J. Mater. Chem. A, 2015, 3, 11700-11715. 184 T. S. Tran, et al., RSC Adv., 2016, 6, 12003-12008. 185 K. R. Paton, et al., Nat. Mater., 2014, 13, 624-630. 186 W. K. Park, et al., Sci. Rep., 2017, 7, 16414. 187 J. Fu, et al., Mater. Res. Innovations, 2015, 19, 278-280. 188 A. V. Talyzin, et al., Carbon, 2011, 49, 1894-1899. 189 I. Ogino, et al., Chem. Mater., 2014, 26, 3334-3339. 190 D. W. Kim, et al., Carbon, 2015, 88, 126-132. 191 S. Tian, et al., Sci. Rep., 2016, 6, 34127.

192 A. A. Green and M. C. Hersam, J. Phys. Chem. Lett., 2010, 1, 544-549.
193 J.-J. Huang and Y. J. Yuan, Phys. Chem. Chem. Phys., 2016, 18, 12312-12322.

194 X. Lin, et al., ACS Nano, 2012, 6, 10708-10719.

195 U. Khan, et al., Carbon, 2012, 50, 470-475.

196 M. K. Brakke, J. Am. Chem. Soc., 1951, 73, 1847-1848.

197 E. Bidram, et al., Carbon, 2016, 103, 363-371.

198 X. Sun, et al., ACS Nano, 2010, 4, 3381-3389.

199 S. Li, et al., J. Electroanal. Chem., 2013, 703, 135-145.

200 X. J. Chen, et al., Adv. Mater., 2015, 27, 3654-3660.

201 H. Geng, et al., J. Am. Chem. Soc., 2017, 139, 12517-12523.

202 H. V. Kumar, et al., Carbon, 2016, 98, 491-495.

203 S. Zhang, et al., RSC Adv., 2016, 6, 74053-74060.

204 C. Le Quéré, et al., Earth Syst. Sci. Data, 2018, 10, 405-448. 205 U.S. Geological Survey, 2017, Mineral commodity summaries 2017, U.S. Geological Survey, 74-75. 